\title{
GENERAL THEORY OF SINGULAR INTEGRAL EQUATIONS WITH REAL KERNELS*
}

\author{
BY \\ W. J. TRJITZINSKY
}

1. Introduction. Amongst the outstanding theories of integral equations of particular importance from our present point of view are those due to Vito Volterra,$\dagger$ I. Fredholm, $\ddagger$ D. Hilbert,$\S$ E. Schmidt, $\|$ and T. Carleman. $\rceil$ With respect to generality these contributions, in the order mentioned, form an ascending hierarchy of theories, with those of Hilbert and Schmidt essentially on the same level, while the developments of Carleman present the culminating aspects. In considering integral equations of the form

$$
\begin{gathered}
\phi(x)-\lambda \int_{a}^{b} K(x, y) \phi(y) d y=f(x), \\
\phi(x)-\lambda \int_{a}^{b} K(x, y) \phi(y) d y=0 \\
{[f(x) \text { given on }(a, b) ; \text { real } K(x, y) \text { given on } a \leqq x, y \leqq b] .}
\end{gathered}
$$

one may, with advantage and without any substantial loss of generality, confine oneself to symmetric kernels $K(x, y)$,

$$
K(x, y)=K(y, x) \text {. }
$$

This can be inferred on the basis of certain considerations of Pérès.**

In the sequel, unless the contrary is stated, all kernels involved will be supposed symmetric. All integrals not in the sense of Stieltjes will be in the sense of Lebesgue.

Whenever $\dagger$

* Presented to the Society, September 7, 1939; received by the editors March 7, 1939.

$\dagger$ An exposition of Volterra's work and of many other developments in the field of integral equations as well as an extensive bibliography can be found in the book by V. Volterra and J. Pérès, Théorie Générale des Fonctionnelles, vol. 1, Paris, 1936.

$\ddagger$ Cf. reference on page 344 of Volterra and Pérès, loc. cit.

$\S \mathrm{D}$. Hilbert, Grundziige einer allgemeinen Theorie der linearen Integralgleichungen, Leipzig and Berlin, 1912.

\|I Cf. reference on page 347 of Volterra and Pérès, loc. cit.

I| T. Carleman, Sur les Équations Intégrales Singulières à Noyau Réel et Symétrique, Uppsala, 1923; T. Carleman, La théorie des équations intégrales singulières et les applications, Annales de l'Institut H. Poincaré, vol. 1, pp. 401-430.

** Cf. the book of Volterra and Pérès, loc. cit., pp. 305-306 and pp. 263-264.

$\dagger$ That is, the integrals $\int_{a}^{b} \int_{a}^{b} K^{2}(x, y) d x d y, \int_{a}^{b} f^{2}(x) d x$ exist. 


$$
K(x, y) \subset L_{2}(\text { in } x, y), \quad f(x) \subset L_{2},
$$

the essential results of the Fredholm theory will hold.*

The results of Hilbert's theory will hold in the essential particulars if

$$
\begin{gathered}
\left.K(x, y) \subset L_{2} \quad \text { (in } y \text {; for almost all } x\right), \\
\int_{a}^{b} \int_{a}^{b} K(x, y) \phi(x) \phi(y) d x d y \leqq k^{2} \int_{a}^{b} \phi^{2}(x) d x
\end{gathered}
$$

( $k$ independent of $\phi(x)$ ).

The highly important investigations of Carleman extend these theories as follows. In some of his investigations (1.4) is assumed (for all $x$ except for $x=\xi_{1}, \xi_{2}, \cdots$; the $\xi_{\nu}$ possessing merely a finite number of limiting points), while condition (1.4a) is deleted; in certain other developments he retains (1.4), deletes (1.4a) and assumes the mean continuity relation

$$
\lim _{x_{1} \rightarrow x_{2}} \int_{a}^{b}\left[K\left(x_{1}, y\right)-K\left(x_{2}, y\right)\right]^{2} d y=0 \quad\left(x_{1}, x_{2} \neq \xi_{1}, \xi_{2}, \cdots\right) .
$$

Carleman also has a still more general theory in which the conditions (1.4), $(1.4 \mathrm{a}),(1.4 \mathrm{~b})$ are deleted and it is merely assumed that $K(x, y)$ is a limit (in the ordinary sense or in the mean square with respect to $y$ ) of kernels satisfying (1.3).

The applications of Carleman's results (or of suitable extensions of them) have been numcrous and important; witness, for instance, the application to the Schrödinger wave equation $\dagger$ and to nonlinear ordinary differential equations (of the type occurring in dynamics). $\neq$

Our object in the present work is to develop a theory of equations (1.1) (with $\left.f(x) \subset L_{2}\right)$, (1.2) with kcrnels $K(x, y)$ which, while not necessarily of Carleman's type, are limits (in one sense or another) of kernels of Carleman's type. The kernels of this description will be said to be of rank two. More generally we shall develop theories of equations whose kernels $K(x, y)$ are of any rank $n(\geqq 2)$. In this connection $K(x, y)$ will be said to be of rank $n$ if $K(x, y)$, while not necessarily of rank $n-1$, is a limit (in a suitable sense) $\$$ of kernels of ranks less than $n$. In accordance with the above, Carleman's kernels are said to be of rank 1 .

* A more precise statement in this regard can be found in Carleman, Annales de l'Institut H. P'oincaré, loc. cit., pp. 401-402.

† T. Carleman, Sur la théoric mathématique de l'équation de Schrödinger, Arkiv för Matematik. Astronomi och Fysik, vol. 2413 (1934), pp. 1-7.

$\ddagger$ T. Carleman, A pplication de la théorie des équations intégrales linéaires aux systimes d'équations différentielles non linécuires, Acta Mathematica, vol. 59, pp. 63-87.

$\$$ More precise formulation will be given in the sequel. 
In these pages we shall consider also equations whose kernels are of transfinite rank.

In the sequel Carleman's book will be referred to as (C).

We shall have occasion to use the following known theorems.

Theorem 1.1. (Helly.) Let $\alpha(x, n)(n=1,2, \cdots)$ be of bounded variation for $a \leqq x \leqq b$. If Var. $\alpha(x, n)<A(n=1,2, \cdots ; A$ independent of $n)$ and if $\lim _{n} \alpha(x, n)=\alpha(x)$, then

$$
\lim _{n} \int_{a}^{b} w(x) d_{x}(x, n)=\int_{a}^{b} w(x) d_{x} \alpha(x) \quad \text { (for } w(x) \text { continuous). }
$$

TheOREM 1.2. (F. Riesz.) Suppose $f_{\nu}(x) \subset L_{2}, g_{\nu}(x) \subset L_{2}$ (for $\nu=1,2, \cdots$ and $x$ on $(a, b)$ ) and $f_{\nu}(x) \rightarrow f(x), g_{\nu}(x) \rightarrow g(x)$ (almost everywhere). Then, provided

$$
\int_{a}^{b} g_{\nu}^{2}(x) d x<c, \quad\left|f_{\nu}(x)\right|<\gamma(x) \subset L_{2} \quad(\nu=1,2, \cdots),
$$

one has

$$
\lim _{n} \int_{a}^{b} f_{n}(x) g_{n}(x) d x=\int_{a}^{b} f(x) g(x) d x .
$$

THEOREM 1.3. (F. Riesz.) If $f_{\nu}(x) \subset L_{2}$ on $(a, b)(\nu=1,2, \cdots)$ and if

$$
\int_{a}^{b} f_{\nu}^{2}(x) d x<M \quad(\nu=1,2, \cdots),
$$

then there exists a subsequence $\left\{f_{v_{j}}(x)\right\}\left(\nu_{1}<\nu_{2}<\cdots\right)$ such that, as $j \rightarrow \infty$, $f_{v_{j}}(x) \rightarrow f(x)$ weakly; that is,

$$
\lim _{j} \int_{a}^{x} f_{v_{j}}(x) d x=\int_{a}^{x} f(x) d x
$$

moreover $\int_{a}^{b} f^{2}(x) d x \leqq M$.

TheOREM 1.4. (F. Riesz.) Let $f_{\nu}(x) \subset L_{2}$ on $(a, b)(\nu=1,2, \cdots)$ and suppose $f_{\nu}(x) \rightarrow f(x)$ weakly; then, provided $g(x) \subset L_{2}$, one has

$$
\lim _{\nu} \int_{a}^{b} f_{\nu}(x) g(x) d x=\int_{a}^{b} f(x) g(x) d x .
$$

TheOREM 1.5. (T. Carleman.*) If. $\int_{a}^{b} f_{\nu}^{2}(x) d x<c, f_{\nu}(x) \rightarrow f(x)$ weakly, $g_{n}(x) \rightarrow g(x)$ and $\left|g_{n}(x)\right|<\gamma(x) \subset L_{2}$, then

$$
\lim _{n} \int_{a}^{b} f_{n}(x) g_{n}(x) d x=\int_{a}^{b} f(x) g(x) d x .
$$

*(C), pp. 132-133. 
Another theorem necessary for our purposes will be the theorem of $(\mathrm{C}, \mathrm{pp}$. $21,22)$, which constitutes an extension by Carleman of a result due to Hilbert. This theorem, in the sequel referred to as the "Compactness Theorem," gives conditions under which there exists a sequence of values $\delta_{r}$ $\left(r=1,2, \cdots ; \delta_{r} \rightarrow 0\right)$ such that

$$
\lim _{\delta_{r}=0} f\left(\lambda, x_{1}, \cdots, x_{n} \mid \delta_{r}\right)=F\left(\lambda, x_{1}, \cdots, x_{n}\right),
$$

where $f\left(\lambda, x_{1}, \cdots, x_{n} \mid \delta\right)$ is a given family of functions defined for $\left(x_{1}, \cdots, x_{n}\right)$ in a domain $D$ for every $\lambda$ on $(\alpha, \beta)$. On account of the length of this theorem the reader will be merely referred to (C, pp. 21, 22).

In the sequel we shall give examples of kernels which come under our classification and which at the same time are not of Carleman's type.

In \$2 (Definition 2.2) will be introduced kernels of finite rank $n$ belonging to classes designated as $H_{n}$. The main results for $K(x, y) \subset H_{n}$ are given in Theorems 4.1, 5.1, 7.1.

In $\$ 10$ (Definition 10.1) will be specified kernels of transfinite ranks $\beta$ ( $\beta$ of the secund class), the results for which will be given in Theorems 11.1, 11.2.

2. Kernels of class $H_{n}$. Let

$$
E=E^{0}=\left(I_{1}, I_{2}, \cdots\right)
$$

be a denumerable set of points on the closed interval $(a, b)$. Let us take $E$ reducible closed with, let us say, the $n$th derived set,

$$
E^{n}=\left(I_{1}^{n}, I_{2}^{n}, \cdots\right)=\left(s_{1}, s_{2}, \cdots, s_{k}\right)
$$

consisting of a finite number of points (with at least one point present). The 1st, 2d, $\cdots,(n-1)$ st derived sets of $E$ will then be denumerable sets

$$
E^{\nu}=\left(I_{1}^{\nu}, I_{2}^{\nu}, \cdots\right) \quad(\nu=1,2, \cdots, n-1),
$$

each actually containing an infinity of points.

Definition 2.1. A set E, given by (2.1) and satisfying the above conditions, will be said to belong to $R_{n}, E \subset R_{n}{ }^{*}$.

Given a set $E \subset R_{n-1}(n \geqq 1)$, we shall form sets of closed intervals

$$
\Delta^{0}\left(\delta_{0}\right), \Delta^{1}\left(\delta_{1}\right), \cdots, \Delta^{n-1}\left(\delta_{n-1}\right)
$$

as follows. The intervals of $\Delta^{0}\left(\delta_{0}\right)$ will be

$$
\Delta_{\nu}^{0}\left(\delta_{0}\right)=\left(s_{\nu}-\delta_{0}, s_{\nu}+\delta_{0}\right) \quad\left[\nu=1,2, \cdots, k ; E^{n-1}=\left(s_{1}, \cdots, s_{k}\right)\right] .
$$

Here and in the sequel the parts of the intervals exterior to $(a, b)$ will be discarded.

* If $E \subset R_{0}, E$ consists of a finite number of points. 
In (2.2) $\delta_{0}(>0)$ will be chosen sufficiently small so that no two intervals of (2.2) will have points in common; moreover, $\delta_{0}$ is to be taken so that no end point of the $\Delta_{\nu}^{0}\left(\delta_{0}\right)$ should be coincident with a point of $E$ (except, perhaps, $a$ or $b$; analogous statements are implied in the sequel).*

With $\delta_{0}(>0)$ chosen as stated above, consider the set

$$
\Gamma\left(\delta_{0}\right)=(a, b)-\sum_{\nu=1}^{k} \Delta_{\nu}^{0}\left(\delta_{0}\right) .
$$

It is open. Hence, since the limiting points $s_{\nu}(\nu=1, \cdots, k)$ of $E^{n-2}$ are all in the intervals (2.2), as specified, we observe that, on one hand, there is only a finite number of points of $E^{n-2}$, let us say

$$
s_{2}^{n-2}, s_{2}^{n-2}, \cdots, s_{m\left(\delta_{0}\right)}^{n-2} \quad\left(m\left(\delta_{0}\right) \rightarrow \infty, \text { as } \delta_{0} \rightarrow 0\right),
$$

in $\Gamma\left(\delta_{0}\right)$ and that, on the other hand, these points $(2.2 \mathrm{~b})$ can be enclosed in closed intervals (whose totality constitutes the set $\Delta^{1}\left(\delta_{1}\right)$ )

$$
\Delta_{\nu}^{1}\left(\delta_{1}\right)=\left(s_{\nu}^{n-2}-\delta_{1}, s_{\nu}^{n-2}+\delta_{1}\right) \quad\left(\nu=1,2, \cdots, m\left(\delta_{0}\right)\right)
$$

so that with $\delta_{1}(>0)$ sufficiently small and suitably chosen the following will be true. The intervals

$$
\Delta_{\nu}{ }^{0}\left(\delta_{0}\right) \quad(\nu=1, \cdots, k), \quad \Delta_{\nu}{ }^{1}\left(\delta_{1}\right) \quad\left(\nu=1, \cdots, m\left(\delta_{0}\right)\right)
$$

are all without common points; moreover, no end point of any interval $\Delta_{\nu}{ }^{1}\left(\delta_{1}\right)$ is coincident with a point of $E$. It is to be noted that the intervals $(2.3 \mathrm{a})$ will certainly be without common points if we take $\delta_{1} \leqq \delta_{1}\left(\delta_{0}\right)$, where $\delta_{1}\left(\delta_{0}\right)(>0)$ is sufficiently small but, generally, depends on $\delta_{0}$.

Suppose $\delta_{0}, \delta_{1}$ chosen as stated above. The set

$$
\Gamma\left(\delta_{0}, \delta_{1}\right)=(a, b)-\sum_{\nu=1}^{k} \Delta_{\nu}{ }^{0}\left(\delta_{0}\right)-\sum_{\nu=1}^{m\left(\delta_{0}\right)} \Delta_{\nu}{ }^{1}\left(\delta_{1}\right)
$$

will be open. An infinity of points of $E^{n-2}$ are in the intervals (2.2); all the other points of $E^{n-2}$ - the points (2.2b) -are in the intervals (2.3); thus, all the limiting points of $E^{n-3}$ are interior points of the closed intervals (2.3a). Consequently there is only a finite number of points of $E^{n-3}$, say

$$
s_{1}^{n-3}, s_{2}^{n-3}, \cdots, s_{m\left(\delta_{0}, \delta_{1}\right)}^{n-3},
$$

in the set $\mathrm{I}\left(\delta_{0}, \delta_{1}\right)(2.4)$. The points $(2.4 \mathrm{a})$ can be enclosed in a set $\Delta^{2}\left(\delta_{2}\right)$ of closed intervals

$$
\Delta_{\nu}^{2}\left(\delta_{2}\right)=\left(s_{\nu}^{n-3}-\delta_{2}, s_{\nu}^{n-3}+\delta_{2}\right) \quad\left(\nu=1,2, \cdots, m\left(\delta_{0}, \delta_{1}\right)\right) .
$$

\footnotetext{
* The point $a$ (or $b)$ will be considered interior to any subinterval $\left(a, a^{\prime}\right)$ (or $\left(b^{\prime}, b\right)$ ) of $(a, b)$.
} 
Taking $0<\delta_{2} \leqq \delta_{2}\left(\delta_{0}, \delta_{1}\right)\left[\delta_{2}\left(\delta_{0}, \delta_{1}\right)\right.$ sufficiently small], with suitable choice of $\delta_{2}$ we secure the following. The intervals

$$
\begin{gathered}
\Delta_{\nu}{ }^{0}\left(\delta_{0}\right) \quad(\nu=1, \cdots, k), \quad \Delta_{\nu}{ }^{1}\left(\delta_{1}\right) \quad\left(\nu=1, \cdots, m\left(\delta_{0}\right)\right), \\
\Delta_{\nu}{ }^{2}\left(\delta_{2}\right) \quad\left(\nu=1, \cdots, m\left(\delta_{0}, \delta_{1}\right)\right)
\end{gathered}
$$

are all without common points; no end point of these intervals is coincident with a point of $E$.

We continue this process a finite number of times, finally constructing the $n$ sets of closed intervals

$$
\Delta^{i}\left(\delta_{i}\right) \quad(i=0,1, \cdots, n-1)
$$

possessing properties of the following description.

The set $\Delta^{i}\left(\delta_{i}\right)$ consists of the intervals

$$
\Delta_{\nu}^{i}\left(\delta_{i}\right)=\left(s_{\nu}^{n-1-i}-\delta_{i}, s_{\nu}^{n-1-i}+\delta_{i}\right) \quad\left[\nu=1,2, \cdots, m\left(\delta_{0}, \cdots, \delta_{i-1}\right)\right]
$$

for $i=1,2, \cdots, n-1$. The set $\Delta^{0}\left(\delta_{0}\right)$ consists of the intervals (2.2). The numbers $\delta_{i}$,

$$
\begin{array}{r}
0<\delta_{i} \leqq \delta_{i}\left(\delta_{0}, \delta_{1}, \cdots, \delta_{i-1}\right) \quad\left(i=1, \cdots, n-1 ; 0<\delta_{0} \leqq \delta^{0} ;\right. \\
\left.\delta_{i}\left(\delta_{0}, \delta_{1}, \cdots, \delta_{i-1}\right), \delta^{0} \text { sufficiently small }\right),
\end{array}
$$

are so chosen that no point of $E((2.1))$ is coincident with an end point of any of the intervals of the sets (2.5) and that all the intervals of the sets (2.5) are without common points. The set

$$
\begin{aligned}
\Gamma\left(\delta_{0}, \delta_{1}, \cdots, \delta_{n-1}\right)= & (a, b)-\sum_{\nu=1}^{k} \Delta_{\nu}{ }^{0}\left(\delta_{0}\right)-\sum_{\nu=1}^{m\left(\delta_{0}\right)} \Delta_{\nu}^{1}\left(\delta_{1}\right) \\
& -\sum_{\nu=1}^{m\left(\delta_{0}, \delta_{1}\right)} \Delta_{\nu}^{2}\left(\delta_{2}\right)-\cdots-\sum_{\nu=1}^{m^{1}} \Delta_{\nu}^{n-1}\left(\delta_{n-1}\right)
\end{aligned}
$$

$\left[m^{1}=m\left(\delta_{0}, \delta_{1}, \cdots, \delta_{n-2}\right)\right]$ is open and contains no points of $E$. The totality of all limiting points of $E^{n-2}$ (that is, $E^{n-1}$ ) consists of the centers of the intervals of $\Delta^{0}\left(\delta_{0}\right)$. The limiting points of $E^{n-3}$ are partly contained in $\Delta^{0}\left(\delta_{0}\right)$ and the rest of them, the points $(2.2 \mathrm{~b})$, are centers of the intervals $\Delta^{1}\left(\delta_{1}\right)$. An infinity of limiting points of $E^{n-4}$ (that is, the points of $E^{n-3}$ ) are in

$$
\Delta^{0}\left(\delta_{0}\right)+\Delta^{1}\left(\delta_{1}\right)
$$

the rest of these limiting points, the points $(2.4 \mathrm{a})$, are the centers of the intervals of $\Delta^{2}\left(\delta_{2}\right)$. In general, an infinity of limiting points of $E^{i-1}$ (that is, points of $\left.E^{i}\right)$ are interior to the set

$$
\Delta^{0}\left(\delta_{0}\right)+\Delta^{1}\left(\delta_{1}\right)+\cdots+\Delta^{n-i-2}\left(\delta_{n-i-2}\right) ;
$$


the rest of the limiting points of $E^{i-1}$, the points

$$
s_{1}^{i}, s_{2}^{i}, \cdots, s_{m^{1}}^{i} \quad\left(m^{1}=m\left(\delta_{0}, \cdots, \delta_{n-i-2}\right)\right),
$$

consist of the centers of the intervals of the set $\Delta^{n-i-1}\left(\delta_{n-i-1}\right)$. In particular, the limiting points of $E=E^{0}(2.1)$, that is, the points $E^{1}$, are distributed as follows. A finite number of points of $E^{1}$,

$$
s_{1}^{1}, s_{2}{ }^{1}, \cdots, s_{m^{1}}^{1} \quad\left(m^{1}=m\left(\delta_{0}, \cdots, \delta_{n-3}\right)\right),
$$

constitute the centers of the intervals of the set $\Delta^{n-2}\left(\delta_{n-2}\right)(2.6)$; all the other points of $E^{1}$ are interior to

$$
\Delta^{0}\left(\delta_{0}\right)+\cdots+\Delta^{n-3}\left(\delta_{n-3}\right) .
$$

In the open set

$$
\Gamma\left(\delta_{0}, \cdots, \delta_{n-2}\right)=(a, b)-\Delta^{0}\left(\delta_{0}\right)-\cdots-\Delta^{n-2}\left(\delta_{n-2}\right)
$$

there is only a finite number of points of $E$, say

$$
s_{1}^{0}, s_{2}^{0}, \cdots, s_{m 1}^{0} \quad\left(m^{1}=m\left(\delta_{0}, \cdots, \delta_{n-2}\right)\right) .
$$

These points (2.9) constitute the centers of the intervals of $\Delta^{n-1}\left(\delta_{n-1}\right)$.

Definition 2.2. Let $E$ be a closed reducible set on the interval $(a, b)$. Suppose $E \subset R_{n-1}$ where $R_{n-1}$ is specified by Definition (2.1). Form sets $\Delta^{i}\left(\delta_{i}\right)$ $(i=0,1, \cdots, n-1)$ of closed intervals (2.6), without common points and covering the set $E$, as described in the text above in connection with (2.2)-(2.9).

$W e$ shall say that a real symmetric kernel $K(x, y) \subset H_{n}$, if

$$
K^{\delta_{0}, \delta_{1}}, \cdots, \delta_{n-1}(x, y) \subset L_{2}
$$

(in $x, y ;$ for $a \leqq x, y \leqq b)$,

the function in the first member of (2.10) being defined as follows:

$$
\begin{aligned}
& K^{\delta_{0}}, \cdots, \delta_{n-1}(x, y)=0 \quad\left[x \text { in } \Delta^{0}\left(\delta_{0}\right)+\Delta^{1}\left(\delta_{1}\right)+\cdots+\Delta^{n-1}\left(\delta_{n-1}\right),\right. \\
& \text { while } a \leqq y \leqq b \text { (or } a \leqq y<x)] \text {; } \\
& K^{\delta_{0}}, \cdots, \delta_{n-1}(x, y)=0 \quad\left[y \text { in } \Delta^{0}\left(\delta_{0}\right)+\Delta^{1}\left(\delta_{1}\right)+\cdots+\Delta^{n-1}\left(\delta_{n-1}\right),\right. \\
& \text { while } a \leqq x \leqq b \text { (or } a \leqq x<y)] \text {; } \\
& \text { (2.11b) } \left.\quad K^{\delta_{0}}, \cdots, \delta_{n-1}(x, y)=K(x, y) \quad \text { [at all other points of } a \leqq x, y \leqq b\right] \text {. }
\end{aligned}
$$

Moreover, this definition will be applied only if (2.10) holds as stated for all admissible* positive values $\delta_{i}(i=0, \cdots, n-1)$ no matter how small.

In conformity with this definition, $K(x, y) \subset H_{0}$ is to imply that

$$
K(x, y) \subset L_{2}
$$

* That is, values $\delta_{i}(i=0, \cdots, n-1)$ such that the italicized statement preceding (2.6b) holds. 
so that in this case $K(x, y)$ will be a kernel for which the results of the Fredholm type will hold. Kernels $K(x, y) \subset H_{1}$ are precisely Carleman's kernels of the type considered in ( $\mathrm{C}$; chap. 4 ). For any $n>1$ it is possible to show that there exist kernels which belong to $H_{n}$ and at the same time do not belong to $H_{n-1}$; we shall give such an example for $n=2$.

The following observations regarding kernels included in $H_{n}$ are in order, it being understood that everywhere in the sequel the values $\delta_{i}(i=0, \cdots, n-1)$ are taken as "admissible" (cf. footnote to Definition 2.2).

The function

$$
\left|K^{\delta_{0}, \delta_{1}}, \cdots, \delta_{n-1}(x, y)\right|
$$

is monotone non-decreasing as $\delta_{n-1} \rightarrow 0$; the limit

$$
\lim _{\delta_{n-1}} K^{\delta_{0}, \delta_{1}}, \cdots, \delta_{n-1}(x, y)=K^{\delta_{0}, \delta_{1}}, \cdots, \delta_{n-2}(x, y)
$$

exists and

$$
\left|K^{\delta_{0}}, \cdots, \delta_{n-1}(x, y)\right| \leqq\left|K^{\delta_{0}, \delta_{1}, \cdots, \delta_{n-2}}(x, y)\right| .
$$

In succession we obtain the limits

$$
\begin{gathered}
\lim _{\delta_{n-2}} K^{\delta_{0}}, \cdots, \delta_{n-2}(x, y)=K^{\delta_{0}} \cdots, \delta_{n-s}(x, y), \cdots, \\
\lim _{\delta_{1}} K^{\delta_{0}, \delta_{1}}(x, y)=K^{\delta_{0}}(x, y), \quad \lim _{\delta_{0}} K^{\delta_{0}}(x, y)=K(x, y) .
\end{gathered}
$$

It is also noted that

$$
\left|K^{\delta_{0}}, \cdots, \delta_{i}(x, y)\right| \leqq\left|K^{\delta_{0}}, \cdots, \delta_{i-1}(x, y)\right|,
$$

and that the first member in this inequality is monotone non-decreasing as $\delta_{i} \rightarrow 0$; this can be asserted for $i=n-1, n-2, \cdots, 0$. In view of (2.13) one may write

$$
K(x, y)=\lim _{\delta_{0}} \lim _{\delta_{1}} \cdots \lim _{\delta_{n-2}} \lim _{\delta_{n-1}} K^{\delta_{0}, \delta_{1}}, \cdots, \delta_{n-1}(x, y),
$$

where the order of the limiting processes, in general, cannot be interchanged. It is also observed that the functions of the second members of (2.12), (2.13) belong to the classes $H_{j}$ as follows

$$
K^{\delta_{0}, \delta_{1}}, \cdots, \delta_{i}(x, y) \subset H_{n-1-i} \quad(i=0,1, \cdots, n-1) .^{*}
$$

The above considerations lead to the conclusion that kernels $K(x, y) \subset H_{n}$ are also of rank $n$, according to the terminology of $\$ 1$.

Example of $K(x, y) \subset H_{2}$, but not belonging to $H_{1}$ (that is, not of Carleman's type). To construct such an example we shall take $a=0, b=1$ and define

\footnotetext{
* As indicated before, the class $H_{0}$ is identical with the class of functions $L_{2}$ (in two variables).
} 
$K(x, y)$ by the relations

$$
\begin{aligned}
& K(x, y)=g(x) \quad(\text { for } 0 \leqq y<x), \\
& K(x, y)=g(y) \\
& \text { (for } 0 \leqq x<y \text { ), }
\end{aligned}
$$

the definition for $y=x$ being immaterial;

$$
\begin{array}{rlrl}
\text { (2.16b) } & g(x) & =g_{\nu}(x) & (1 /(\nu+1)<x<1 / \nu ; \nu=1,2, \cdots), \\
(2.16 \mathrm{c}) & g_{\nu}(x) & =0 & \left(1 /(\nu+1)<x<\gamma_{\nu}=(2 \nu+1) /(2 \nu(\nu+1))\right), \\
g_{\nu}(x) & =\frac{c_{\nu}^{1 / 2}}{\left(\nu^{-2}-x^{2}\right)^{1 / 2}} & \left(\gamma_{\nu} \leqq x<1 / \nu ; c_{\nu}>0\right) .
\end{array}
$$

For this kernel the set $E((2.1))$ consists of the points $0,1 / \nu(\nu=1,2, \cdots)$; the derived set $E^{1}((2.1 \mathrm{a}))$ will be $E^{1}=\left(s_{1}\right)\left(s_{1}=0\right)$. Thus $E \subset R_{1}$ (Definition 2.1). The set $\Delta^{0}\left(\delta_{0}\right)$ will consist of a single interval (cf. (2.2))

$$
\Delta_{1}^{0}\left(\delta_{0}\right)=\left(0, \delta_{0}\right)
$$$$
\left(0<\delta_{0}<1\right) \text {, }
$$

where $\delta_{0} \neq 1 / i(i=1,2, \cdots)$. For some integer $m\left(\delta_{0}\right)$

$$
1 /\left(m\left(\delta_{0}\right)+1\right)<\delta_{0}<1 /\left(m\left(\delta_{0}\right)\right) .
$$

The set $\Delta^{1}\left(\delta_{1}\right)$ will consist of the intervals (cf. (2.3))

$$
\Delta_{\nu}{ }^{1}\left(\delta_{1}\right)=\left(1 / \nu-\delta_{1}, 1 / \nu+\delta_{1}\right) \quad\left(\nu=1,2, \cdots, m\left(\delta_{0}\right)\right),
$$

where $0<\delta_{1} \leqq \delta_{1}\left(\delta_{0}\right)$ with $\delta_{1}\left(\delta_{0}\right)$ denoting a positive number less than each of the two numbers

$$
1 /\left(2 m\left(\delta_{0}\right)\left(m\left(\delta_{0}\right)-1\right)\right), 1 /\left(m\left(\delta_{0}\right)\right)-\delta_{0} .
$$

Then, by (2.11), (2.11a) and (2.11b), we have for $y<x$

$$
\begin{aligned}
& K^{\delta_{0}, \delta_{1}}(x, y)=0 \\
& {\left[x \text { in } \Delta_{1}{ }^{0}\left(\delta_{0}\right), \Delta_{\nu}{ }^{1}\left(\delta_{1}\right)\left(\nu=1, \cdots, m\left(\delta_{0}\right)\right) ; \text { cf. }(2.17),(2.17 \mathrm{~b})\right] ;} \\
& K^{\delta_{0}, \delta_{1}}(x, y)=g(x) \quad\left[x \text { in }(0,1)-\Delta^{0}\left(\delta_{0}\right)-\sum_{\nu} \Delta_{\nu}{ }^{1}\left(\delta_{1}\right)\right] ;
\end{aligned}
$$

for $x>y$ the function of the first member of (2.18) is defined by symmetry. Whence by virtue of $(2.16 \mathrm{~b}),(2.16 \mathrm{c})$, it is inferred that

$$
\begin{aligned}
\int_{0}^{1} \int_{0}^{1}\left|K^{\delta_{0}, \delta_{1}}(x, y)\right|^{2} d x d y= & 2 \int_{x=\delta_{0}}^{1 / m\left(\delta_{0}\right)-\delta_{1}} \int_{y=0}^{x} g_{m\left(\delta_{0}\right)}^{2}(x) d y d x \\
& +\sum_{\nu=1}^{m\left(\delta_{0}\right)-1} 2 \int_{x=1 /(\nu+1)+\delta_{1}}^{1 / \nu-\delta_{1}} \int_{y=0}^{x} g_{\nu}^{2}(x) d y d x,
\end{aligned}
$$

where 


$$
\begin{aligned}
2 \int_{x=1 /(\nu+1)+\delta_{1}}^{1 / \nu-\delta_{1}} \int_{\nu=0}^{x} g_{\nu}^{2}(x) d y d x=-\lambda_{\nu}+\log T^{-1}\left(\nu, \delta_{1}\right) \\
\quad\left[\lambda_{\nu}=-c_{\nu} \log \left(\nu^{-2}-\gamma_{\nu}^{2}\right) ; T\left(\nu, \delta_{1}\right)=\left(2 \delta_{1} / \nu-\delta_{1}^{2}\right)^{c_{\nu}}\right] .
\end{aligned}
$$

Hence, for all admissible $\delta_{0}(>0)$,

$$
\iint\left|K^{\delta_{0}, \delta_{1}}(x, y)\right|^{2} d x d y \rightarrow+\infty \quad\left(\text { as } \delta_{1} \rightarrow 0\right),
$$

and clearly $K^{\delta_{0}}(x, y)$ does not belong to $L_{2}$ (in $x, y$ ). Consequently it is clear that $K(x, y)$, as given by (2.16)-(2.16c), is a kernel satisfying the conditions of the italicized statement preceding (2.16). It is essential to note that for the example considered above the integral

$$
\int_{0}^{1} K^{2}(x, y) d y
$$

diverges; in fact, convergence of this integral would have meant that $K(x, y)$ is essentially of Carleman's type.*

Some of the developments for integral equations whose kernels are included in $H_{n}$ will be given with the aid of operators $L$ specified as follows.

Definition 2.3. Given a kernel $K(x, y) \subset H_{n}$ (Definition 2.2), a linear operator $L_{x}(\xi \mid h(x)$ ) ( $\xi$ a parameter) will be said to be associated with $K(x, y)$ if

$$
\begin{gathered}
L_{x}(\xi \mid K(x, y)) \subset L_{2} \\
\left|L_{x}\left(\xi \mid K^{\delta_{0}, \delta_{1}}, \cdots, \delta_{n-1}(x, y)\right)\right|<\gamma(\xi \mid y),
\end{gathered}
$$

where $\gamma(\xi \mid y) \subset L_{2}$ (in $y$ ) and $\gamma(\xi \mid y)$ is independent of $\delta_{0}, \delta_{1}, \ldots, \delta_{n-1}$;

$$
\begin{aligned}
\lim _{\delta_{n-1}} L_{x}\left(\xi \mid K^{\delta_{0}}, \cdots, \delta_{n-1}(x, y)\right) & =L_{x}\left(\xi \mid K^{\delta_{0}} \cdots, \delta_{n-2}(x, y)\right), \\
\lim _{\delta_{n-2}} L_{x}\left(\xi \mid K^{\delta_{0}} \cdots, \delta_{n-2}(x, y)\right) & =L_{x}\left(\xi \mid K^{\delta_{0}} \cdots, \delta_{n-3}(x, y)\right), \cdots, \\
\lim _{\delta_{0}} L_{x}\left(\xi \mid K^{\delta_{0}}(x, y)\right) & =L_{x}(\xi \mid K(x, y))
\end{aligned}
$$

whenever $f_{\nu}(x) \subset L_{2}$ converges weakly $($ as $\nu \rightarrow \infty)$ to $f(x)(a \leqq x \leqq b)$ we have

$$
\lim _{\nu} L_{x}\left(\xi \mid f_{\nu}(x)\right)=L_{x}(\xi \mid f(x))
$$

$$
\int_{a}^{b} L_{x}\left(\xi \mid K^{\delta_{0}}, \cdots, \delta_{n-1}(x, y)\right) \phi(y) d y=L_{x}\left(\xi \mid \int_{a}^{b} K^{\delta_{0}}, \cdots, \delta_{n-1}(x, y) \phi(y) d y\right),
$$

whenever $\phi(x) \subset L_{2}$.

\footnotetext{
* This follows by Carleman, Annales de l'Institut H. Poincaré, loc. cit.
} 
Note. For $n=1$ an operator described in the above definition reduces precisely to the operator $L$ given in (C, pp. 137, 138).

In order to make certain that those of the developments, with respect to kernels included in $H_{n}(n>1)$, which are made with the aid of operators $L$ (Definition 2.3) should have a significance, it is essential to show the following.

There exist kernels $K(x, y)$, included in $H_{n}(n>1)$ and not belonging to $H_{n-1}$, with which one can associate an operator $L$ satisfying the conditions of Definition 2.3.

We shall give such an example for $n=2$. For $n>2$ similar examples can be given following similar procedures. ${ }^{*}$ It will be sufficient to construct an operator $L$ associated with the kernel $K(x, y)$, given by (2.16)-(2.16c). Let us take

$$
L_{x}(\xi \mid h(x))=\int_{0}^{1} G(\xi \mid x) h(x) d x,
$$

where, for $\nu=1,2, \cdots$,

$$
\begin{array}{ll}
G(\xi \mid x)=G_{\nu}(\xi \mid x) \quad\left(\text { for } \gamma_{\nu} \leqq\right. & \left.x<1 / \nu ; \gamma_{\nu} \text { from }(2.16 \mathrm{c})\right) \\
G(\xi \mid x)=-G_{\nu}\left(\xi \mid 2 \gamma_{\nu}-x\right) & \left(\text { for } 1 /(\nu+1)<x \leqq \gamma_{\nu}\right) ; \dagger
\end{array}
$$

here we take

$$
G_{\nu}(\xi \mid x)=c_{\nu}^{-1 / 2}\left(\nu^{-2}-x^{2}\right)^{1 / 2} w_{\nu}(\xi \mid x), \quad 0 \leqq w_{\nu}(\xi \mid x) \leqq H,
$$

where $\theta$ is independent of $\nu, x$ and $w_{\nu}(\xi \mid x)$ [included in $L_{1}$ in $x$ on $\left(\gamma_{\nu}, 1 / \nu\right)$ ] is monotone non-increasing in $x$ on $\left(\gamma_{\nu}, 1 / \nu\right)$. Moreover, the $c_{\nu}$ will be taken subject to the requirement that the series

$$
\sum_{\nu} \cdot \frac{1}{c_{\nu} \nu^{5}}
$$

be convergent. We shall now demonstrate that the operator $L_{x}(\xi \mid h(x))$ $((2.25))$, so defined, satisfies the conditions (2.20)-(2.24) with respect to the kernel $K(x, y)[(2.16)-(2.16 \mathrm{c})]$.

By $(2.25 \mathrm{c})$

$$
\left|G_{\nu}(\xi \mid x)\right|^{2} \leqq \frac{H^{2}}{c_{\nu}}\left(\frac{1}{\nu^{2}}-\gamma_{\nu}^{2}\right)<\frac{H^{2}}{c_{\nu} \nu^{3}} \quad\left(\gamma_{\nu} \leqq x<1 / \nu\right) ;
$$

thus, in view of $(2.25 a)$ and $(2.25 b)$,

$$
|G(\xi \mid x)|^{2}<\frac{H^{2}}{c_{\nu} \nu^{3}} \quad(1 /(\nu+1)<x<1 / \nu ; \nu=1,2, \cdots) .
$$

* This will not be done in these pages in order to save space.

$\dagger \gamma_{\nu}$ bisects the interval $(1 /(\nu+1), 1 / \nu) ;(2.25 \mathrm{~b})$ implies symmetry of $G(\xi \mid x)$ with respect to $\gamma_{\nu}$, as indicated. 
Hence

$$
\begin{aligned}
\int_{0}^{1}|G(\xi \mid x)|^{2} d x & =\sum_{\nu=1}^{\infty} \int_{1 /(\nu+1)}^{1 / \nu}|G(\xi \mid x)|^{2} d x \\
& <H^{2} \sum_{\nu=1}^{\infty} \frac{1}{c_{\nu} \nu^{3}}\left(\frac{1}{\nu}-\frac{1}{\nu+1}\right)<H^{2} \sum_{\nu} \frac{1}{c_{\nu} \nu^{5}}
\end{aligned}
$$

the series last displayed being convergent in view of (2.26), it is concluded that

$$
G(\xi \mid x) \subset L_{2} \quad(\text { in } x ; 0 \leqq x \leqq 1) .
$$

By virtue of (2.16), (2.16a) and (2.25)

$$
L_{x}(\xi \mid K(x, y))=\beta(\xi \mid y)+\alpha(\xi \mid y),
$$

where

(2.29a) $\beta(\xi \mid y)=g(y) \int_{x=0}^{y} G(\xi \mid x) d x, \quad \alpha(\xi \mid y)=\int_{y}^{1} G(\xi \mid x) g(x) d x$. By (2.16b), (2.16c)

$$
\beta(\xi \mid y)=0 \quad\left(\text { for } 1 /(\nu+1)<y<\gamma_{\nu}\right) .
$$

Now suppose

$$
\gamma_{\nu} \leqq y<\frac{1}{\nu}
$$

then by (2.16c) from (2.29a) we deduce

$$
\beta(\xi \mid y)=\frac{c_{\nu}^{1 / 2}}{\left(\nu^{-2}-y^{2}\right)^{1 / 2}}\left[\sum_{i=\nu+1}^{\infty} \int_{1 /(i+1)}^{1 / i} G(\xi \mid x) d x+\int_{1 /(\nu+1)}^{\nu} G(\xi \mid x) d x\right] ;
$$

in view of (2.25a) and (2.25b)

whence

$$
\int_{1 /(i+1)}^{1 / i} G(\xi \mid x) d x=0 \quad(i=1,2, \cdots) ;
$$

$$
\begin{aligned}
\beta(\xi \mid y) & =\frac{c_{\nu}^{1 / 2}}{\left(\nu^{-2}-y^{2}\right)^{1 / 2}} \int_{1 /(\nu+1)}^{y} G(\xi \mid x) d x=\frac{c_{\nu}^{1 / 2}}{\left(\nu^{-2}-y^{2}\right)^{1 / 2}} \int_{1 /(\nu+1)}^{2 \gamma_{\nu}-y} G(\xi \mid x) d x \\
& =-\frac{c_{\nu}^{1 / 2}}{\left(\nu^{-2}-y^{2}\right)^{1 / 2}} \int_{1 /(\nu+1)}^{2 \gamma_{\nu}-y} G_{\nu}\left(\xi \mid 2 \gamma_{\nu}-x\right) d x \\
& =-\frac{c_{\nu}^{1 / 2}}{\left(\nu^{-2}-y^{2}\right)^{1 / 2}} \int_{\nu}^{1 / \nu} G_{\nu}(\xi \mid u) d u
\end{aligned}
$$


in view of $(2.25 \mathrm{c})$ and in consequence of the monotone character of $w_{\nu}(\xi \mid u)$ it is concluded that, under (2.31), the integrand last displayed satisfies the inequality $0 \leqq G_{\nu}(\xi \mid u) \leqq G_{\nu}(\xi \mid y)(y \leqq u \leqq 1 / \nu)$; thus, by (2.25c)

$$
|\beta(\xi \mid y)| \leqq \frac{c_{\nu}^{1 / 2}}{\left(\nu^{-2}-y^{2}\right)^{1 / 2}} G_{\nu}(\xi \mid y)\left(\frac{1}{\nu}-y\right) \leqq\left(\frac{1}{\nu}-y\right) H<\frac{H}{2 \nu^{2}}
$$

(under (2.31)).

Inasmuch as (2.30), (2.32) hold for $\nu=1,2, \cdots$, it is inferred that

$$
|\beta(\xi \mid y)|<H / 2
$$$$
(0 \leqq y \leqq 1) \text {. }
$$

On turning attention to $\alpha(\xi \mid y)((2.29 \mathrm{a}))$ it is found that

$$
\begin{aligned}
|\alpha(\xi \mid y)| \leqq \alpha(\xi) & =\int_{0}^{1}|G(\xi \mid x)| g(x) d x \\
& =\sum_{\nu=1}^{\infty} \int_{1 /(\nu+1)}^{1 / \nu}|G(\xi \mid x)| g_{\nu}(x) d x ;
\end{aligned}
$$

by $(2.16 \mathrm{c}),(2.25 \mathrm{a})$ and $(2.25 \mathrm{c})$

(2.34a) $\quad \alpha(\xi)=\sum_{\nu=1}^{\infty} \int_{\gamma_{\nu}}^{1 / \nu}\left|G_{\nu}(\xi \mid x)\right| g_{\nu}(\xi \mid x) d x=\sum_{\nu=1}^{\infty} \int_{\gamma_{\nu}}^{1 / \nu} w_{\nu}(\xi \mid x) d x<H$.

By virtue of (2.33), (2.34), (2.34a), on taking account of (2.29) it is deduced that (2.20) is satisfied for the example under consideration.

We shall now proceed to establish (2.21) (with $n=2$ ). By definition of $K^{\delta_{0}, \delta_{1}}(x, y)[(2.18),(2.18 \mathrm{a})]$

$$
L_{x}\left(\xi \mid K^{\delta_{0}, \delta_{1}}(x, y)\right)=\beta^{\delta_{0}, \delta_{1}}(\xi \mid y)+\alpha^{\delta_{0}, \delta_{1}}(\xi \mid y),
$$

where

$$
\beta^{\delta_{0}, \delta_{1}}(\xi \mid y)=g^{\delta_{0}, \delta_{1}}(y) \int_{x=0}^{y} G(\xi \mid x) d x
$$

$$
\begin{array}{rlrl}
\alpha^{\delta_{0}, \delta_{1}}(\xi \mid y) & =\int_{\nu}^{1} G(\xi \mid x) g^{\delta_{0}, \delta_{1}}(x) d x ; & \\
g^{\delta_{0}, \delta_{1}}(x)=0 & {\left[\text { for } 0 \leqq x \leqq \delta_{0} ; \text { for } x\right. \text { on closed intervals }} \\
\left.\left(1 / \nu-\delta_{1}, 1 / \nu+\delta_{1}\right)\left(\nu=1,2, \cdots, m\left(\delta_{0}\right)\right)\right] ; & {\left[1 /(\nu+1)+\delta_{1}<x<1 / \nu-\delta_{1} ;\right.} \\
g^{\delta_{0}, \delta_{1}}(x)=g_{\nu}(x) & \left.\nu=1,2, \cdots, m\left(\delta_{0}\right)-1 ; \text { cf. }(2.16 c)\right] ; \\
g^{\delta_{0}, \delta_{1}}(x)=g_{m\left(\delta_{0}\right)}(x) & {\left[\delta_{0}<x<1 / m\left(\delta_{0}\right)-\delta_{1}\right] .}
\end{array}
$$


Clearly

$$
0 \leqq g^{\delta_{0}, \delta_{1}}(x) \leqq g^{\delta_{0}}(x) \leqq g(x) \quad(0 \leqq x \leqq 1)
$$

here

$$
g^{\delta_{0}}(x)=\lim _{\delta_{1}} g^{\delta_{0}, \delta_{1}}(x), \quad g(x)=\lim _{\delta_{0}} g^{\delta_{0}}(x) .
$$

By (2.35a) and (2.36) in view of (2.29a) and (2.33)

$$
\left|\beta^{\delta_{0}, \delta_{1}}(\xi \mid y)\right| \leqq g(y)\left|\int_{x=0}^{y} G(\xi \mid x) d x\right|=|\beta(\xi \mid y)|<\frac{H}{2} .
$$

On the other hand, in consequence of (2.35a), (2.36), (2.34) and (2.34a),

$$
\left|\alpha^{\delta_{0}, \delta_{1}}(\xi \mid y)\right| \leqq \int_{y}^{1}|G(\xi \mid x)| g^{\delta_{0}, \delta_{1}}(x) d x \leqq \int_{y}^{1}|G(\xi \mid x)| g(x) d x<H .
$$

In view of (2.35), (2.37) and (2.37a) it is inferred that condition (2.21) (Definition 2.3) holds with $\gamma(\xi \mid y)=3 H / 2$.

To demonstrate the first one of the relations (2.22) it is sufficient to prove that

$$
\lim _{\delta_{1}} \beta^{\delta_{0}, \delta_{1}}(\xi \mid y)=\beta^{\delta_{0}}(\xi \mid y), \quad \lim _{\delta_{1}} \alpha^{\delta_{0}, \delta_{1}}(\xi \mid y)=\alpha^{\delta_{0}}(\xi \mid y),
$$

where

(2.38a) $\quad \beta^{\delta_{0}}(\xi \mid y)=g^{\delta_{0}}(y) \int_{x=0}^{y} G(\xi \mid x) d x, \quad \alpha^{\delta_{0}}(\xi \mid y)=\int_{y}^{1} G(\xi \mid x) g^{\delta_{0}}(x) d x$, with $g^{\delta_{0}}(y)$ denoting the first function displayed in (2.36a),

$$
g^{\delta_{0}}(x)=0 \quad\left(0 \leqq x \leqq \delta_{0}\right), \quad g^{\delta_{0}}(x)=g(x) \quad\left(\delta_{0}<x \leqq 1\right) .
$$

The first of the equalities (2.38) follows immediately from the first relations in (2.35a) and (2.36a). To justify the second relation in (2.38) it is sufficient to show that

$$
\lim _{\delta_{1}} \int_{y}^{1} G(\xi \mid x) g^{\delta_{0}, \delta_{1}}(x) d x=\int_{y}^{1} G(\xi \mid x) g^{\delta_{0}}(x) d x .
$$

The passage to the limit under the integral sign is here justified because the integrand displayed in the first member converges to the integrand displayed in the second member while, as follows by (2.36),

$$
\left|G(\xi \mid x) g^{\delta_{0}, \delta_{1}}(x)\right| \leqq|G(\xi \mid x)| g(x) \subset L_{1} \quad \text { (in } x ; \text { cf. (2.34), (2.34a)). }
$$


The second one of the relations (2.22) will certainly hold if

$$
\lim _{\delta_{0}} \beta^{\delta_{0}}(\xi \mid y)=\beta(\xi \mid y), \quad \lim _{\delta_{0}} \alpha^{\delta_{0}}(\xi \mid y)=\alpha(\xi \mid y),
$$

where $\beta^{\delta_{0}}(\xi \mid y), \alpha^{\delta_{0}}(\xi \mid y)$ are given by (2.38a) and $\beta(\xi \mid y), \alpha(\xi \mid y)$ are the functions of $(2.29 \mathrm{a})$. The first of the equalities (2.39) is a consequence of the last one of (2.36a). The other equality of (2.39), that is the relation

$$
\lim _{\delta_{0}} \int_{y}^{1} G(\xi \mid x) g^{\delta_{0}}(x) d x=\int_{y}^{1} G(\xi \mid x) g(x) d x,
$$

is seen to be true in view of (2.36a) and of the inequality

$$
\left|G(\xi \mid x) g^{\delta_{0}}(x)\right| \leqq|G(\xi \mid x)| g(x) \subset L_{1}
$$

which is deduced from (2.36).

Accordingly it can be asserted that conditions (2.22) of Definition 2.3 all hold for the case under consideration.

The condition stated in connection with (2.23) will hold for all sequences $\left\{f_{\nu}(x)\right\}$ therein specified, since

$$
\lim _{\nu} \int_{0}^{1} G(\xi \mid x) f_{\nu}(x) d x=\int_{0}^{1} G(\xi \mid x) f(x) d x ;
$$

in fact, passage to the limit under the integral sign is here justified in view of (2.28) and of Theorem 1.4.

It remains to verify whether (2.24) holds, that is whether we have

$$
\begin{aligned}
& \int_{y=0}^{1}\left[\int_{x=0}^{1} G(\xi \mid x) K^{\delta_{0}, \delta_{1}}(x, y) d x\right] \phi(y) d y \\
&=\int_{x=0}^{1} G(\xi \mid x)\left[\int_{y=0}^{1} K^{\delta_{0}, \delta_{1}}(x, y) \phi(y) d y\right] d x
\end{aligned}
$$

for all $\phi(y) \subset L_{2}$. The indicated change of order of integration can be justified without difficulty.

The developments from (2.25) to (2.40) enable us to conclude that the kernel $K(x, y)$, as given by (2.16)-(2.16c) and with the $c_{\nu}(>0)$ such that the series (2.26) converges, has associated with it an operator $L$ (cf. (2.25)(2.25c)) satisfying the conditions of Definition 2.3.

3. Formulation of induction for classes $H_{n}$. With $K(x, y) \subset H_{n}$ (Definition 2.2) and $K^{\delta_{0}} \cdots, \delta_{n-1}(x, y)$ being the function specified by (2.11), (2.11a), (2.11b), consider equations

$$
\phi^{\delta_{0}}, \cdots, \delta_{n-1}(x)-\lambda \int_{a}^{b} K^{\delta_{0}}, \cdots, \delta_{n-1}(x, y) \phi^{\delta_{0}, \cdots, \delta_{n-1}}(y) d y=f(x) \quad\left(f(x) \subset L_{2}\right),
$$




$$
\phi^{\delta_{0}}, \cdots, \delta_{n-1}(x)-\lambda \int_{a}^{b} K^{\delta_{0}}, \cdots, \delta_{n-1}(x, y) \phi^{\delta_{0}}, \cdots, \delta_{n-1}(y) d y=0 .
$$

By (2.10) the kernel in (3.1), (3.2) belongs to $H_{0}$ and is thus essentially a Fredholm kernel. In accordance with known facts regarding such equations, the spectrum of the kernel displayed in (3.2) is the function

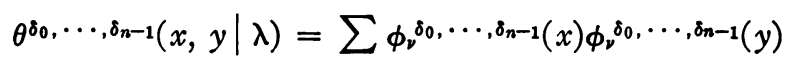

$\left(\lambda>0\right.$; summation over values $\nu$ such that $\left.0<\lambda_{\nu} \delta_{0}, \cdots, \delta_{n-1}<\lambda\right)$;

$$
\begin{gathered}
\theta^{\delta_{0}}, \cdots, \delta_{n-1}(x, y \mid 0)=0 ; \\
\theta^{\delta_{0}}, \cdots, \delta_{n-1}(x, y \mid \lambda)=-\sum \phi_{\nu}^{\delta_{0}} \cdots, \delta_{n-1}(x) \phi_{\nu}^{\delta_{0}} \cdots, \delta_{n-1}(y)
\end{gathered}
$$

$\left(\lambda<0\right.$; summation over values $\nu$ such that $\left.\lambda \leqq \lambda_{\nu}{ }^{\delta_{0}}, \cdots, \delta_{n-1}<0\right)$.

Here the sequence

$$
\left\{\phi_{\nu}^{\delta_{0}}, \cdots, \delta_{n-1}(x)\right\}
$$

forms an orthogonal normal set. The $\lambda_{\nu}{ }^{\delta_{0}}, \cdots, \delta_{n-1}$ are the characteristic values of (3.2); thus

$$
\phi_{\nu}^{\delta_{0}}, \cdots, \delta_{n-1}(x)=\lambda_{\nu}^{\delta_{0}}, \cdots, \delta_{n-1} \int_{a}^{b} K^{\delta_{0}}, \cdots, \delta_{n-1}(x, y) \phi_{\nu}^{\delta_{0}} \cdots, \delta_{n-1}(y) d y .^{*}
$$

By induction we shall establish that certain facts, to be stated explicitly in the remainder of this section, hold for all integral equations (1.1), (1.2) whose kernels are included in $H_{m}$, where $m$ is any finite integer $(\geqq 0)$. Thus, assume that the following facts, stated throughout the rest of this section, hold for kernels included in $H_{n}(n=1, \cdots, m-1)$. An examination of these statements leads to the conclusion that they certainly hold true for $m=2$; that is, for Carleman kernels $H_{1}$; this can be asserted on the basis of (C; chap. 4). In subsequent sections these facts will be shown to hold for $n=m$; which will complete the induction.

Form the function

$$
\int_{a}^{x} \int_{a}^{y} \theta^{\delta_{0}}, \cdots, \delta_{n-1}(x, y \mid \lambda) d x d y=\Omega^{\delta_{0}}, \cdots, \delta_{n-1}(x, y \mid \lambda)(\mathrm{cf} .(3.3)-(3.3 \mathrm{~b})) .
$$

Subsequences of positive numbers

$$
\delta_{n-1, r}(r=1,2, \cdots), \delta_{n-2, r}(r=1,2, \cdots), \cdots, \delta_{0, r}(r=1,2, \cdots)
$$

can be found so that

$$
\lim _{r} \delta_{n-1, r}=0, \cdots, \lim _{r} \delta_{0, r}=0,
$$

* Many properties of $\theta^{\delta 0}, \ldots, \delta_{n-1}(x, y \mid \lambda)$ can be inferred from (C). 
and so that the limits

$$
\begin{aligned}
\lim _{\delta_{n-1, r}} \Omega^{\delta_{0}}, \cdots, \delta_{n-r}(x, y \mid \lambda) & =\Omega^{\delta_{0}} \cdots, \delta_{n-2}(x, y \mid \lambda), \\
\lim _{\delta_{n-2, r}} \Omega^{\delta_{0}}, \cdots, \delta_{n-2}(x, y \mid \lambda) & =\Omega^{\delta_{0}} \cdots, \delta_{n-3}(x, y \mid \lambda), \cdots, \\
\lim _{\delta_{0, r}} \Omega^{\delta_{0}, r}(x, y \mid \lambda) & =\Omega(x, y \mid \lambda)
\end{aligned}
$$

exist for all $(x, y, \lambda),{ }^{*}$ convergence to the limits (3.6) being uniform with respect to $(x, y)$;

$$
\begin{aligned}
\operatorname{Var} . \Omega(x, y \mid \lambda) & \leqq[(x-a)(y-a)]^{1 / 2} ; \dagger \quad \Omega(x, y \mid 0)=0 \\
\left|\Omega\left(x^{\prime}, y^{\prime} \mid \lambda\right)-\Omega(x, y \mid \lambda)\right| & \leqq\left[(b-a)\left|y^{\prime}-y\right|\right]^{1 / 2}+\left[(b-a)\left|x^{\prime}-x\right|\right]^{1 / 2} .
\end{aligned}
$$

The function $\Omega(x, y \mid \lambda)$ may be discontinuous in $\lambda$ for certain values of $\lambda$, say $\lambda_{1}, \lambda_{2}, \cdots$.

We have

$$
\int_{a}^{b}\left|\frac{\partial}{\partial y} \Omega(x, y \mid \lambda)\right|^{2} d y \leqq x-a
$$

[integrand exists for almost all $y ; a \leqq y \leqq b]$.

With the numbers (3.5) suitably chosen one has

$$
\begin{aligned}
\lim _{r} \frac{\partial}{\partial y} \Omega^{\delta_{0}}, \cdots, \delta_{n-1, r}(x, y \mid \lambda) & =\frac{\partial}{\partial y} \Omega^{\delta_{0}, \cdots, \delta_{n-2}}(x, y \mid \lambda), \\
\lim _{r} \frac{\partial}{\partial y} \Omega^{\delta_{0}}, \cdots, \delta_{n-2, r}(x, y \mid \lambda) & =\frac{\partial}{\partial y} \Omega^{\delta_{0}, \cdots, \delta_{n-3}}(x, y \mid \lambda), \cdots, \\
\lim _{r} \frac{\partial}{\partial y} \Omega^{\delta_{0}, r}(x, y \mid \lambda) & =\frac{\partial}{\partial y} \Omega(x, y \mid \lambda),
\end{aligned}
$$

convergence being in the weak sense in $y(a \leqq y \leqq b) . \ddagger$ Also

$$
\int_{a}^{b}\left|\frac{\partial}{\partial x} \int_{y=a}^{b} h(y) \frac{\partial}{\partial y} \Omega(x, y \mid \lambda) d y\right|^{2} d x \leqq \int_{a}^{b} h^{2}(x) d x
$$

* That is, "in general" for $a \leqq x, y \leqq b$ and for all real $\lambda$.

$\dagger$ "Var." means "variation with respect to $\lambda$ " for $(-\infty,+\infty)$ unless the interval is indicated explicitly.

$\ddagger(3.8)$ is assumed for kernels of classes $H_{1}, H_{2}, \cdots, H_{m-1}$; in particular (3.8) will hold for the second members of (3.8a), which are defined for almost all $y$ on $(a, b)$. 
whenever $h(x) \subset L_{2}$; moreover,

$$
\lim _{r} \frac{\partial}{\partial x} \int_{y=a}^{b} h(y) \frac{\partial}{\partial y} \Omega^{\delta_{0}, r}(x, y \mid \lambda) d y=\frac{\partial}{\partial x} \int_{y=a}^{b} h(y) \frac{\partial}{\partial y} \Omega(x, y \mid \lambda) d y,
$$

convergence being reak in $x$ (for a suitable sequence $\delta_{0, r}$ ).

Whenever $g(x), h(y) \subset L_{2}$ the following relations will hold (provided the $\delta_{0, r}$ are suitably chosen):

$$
\begin{aligned}
\lim _{r} \int_{a}^{b} g(x)\left[\frac{\partial}{\partial x} \int_{y=a}^{b} h(y)\right. & \left.\frac{\partial}{\partial y} \Omega^{\delta_{0}, r}(x, y \mid \lambda) d y\right] d x \\
& =\int_{a}^{b} g(x)\left[\frac{\partial}{\partial x} \int_{a}^{b} h(y) \frac{\partial}{\partial y} \Omega(x, y \mid \lambda) d y\right] d x ; \\
\mid \int_{a}^{b} g(x)\left[\frac{\partial}{\partial x} \int_{a}^{b} h(y) \frac{\partial}{\partial y}\right. & \Omega(x, y \mid \lambda) d y]\left.d x\right|^{1 / 2}\left[\int_{a}^{b} g^{2}(x) d x\right]^{1 / 2} ;
\end{aligned}
$$

$$
\begin{aligned}
\text { Var. } \int_{a}^{b} g(x)\left[\frac{\partial}{\partial x} \int_{a}^{b} h(y)\right. & \left.\frac{\partial}{\partial y} \Omega(x, y \mid \lambda) d y\right] d x \\
& \leqq \text { second member above. }
\end{aligned}
$$

Whenever $\alpha(\lambda)$ is continuous on $\left(\lambda_{1}, \lambda_{2}\right)$ and $g(x), h(y) \subset L_{2}$

$$
\begin{aligned}
\int_{\lambda_{1}}^{\lambda_{2}} \alpha(\lambda) d_{\lambda} \int_{a}^{b} g(x) & {\left[\frac{\partial}{\partial x} \int_{a}^{b} h(y) \frac{\partial}{\partial y} \Omega^{\delta_{0}, r}(x, y \mid \lambda) d y\right] d x } \\
& \rightarrow \int_{\lambda_{1}}^{\lambda_{2}} \alpha(\lambda) d_{\lambda} \int_{a}^{b} g(x)\left[\frac{\partial}{\partial x} \int_{a}^{b} h(y) \frac{\partial}{\partial y} \Omega(x, y \mid \lambda) d y\right] d x
\end{aligned}
$$

(as $r \rightarrow 0$; suitable $\delta_{0, r}$ ).

With $\alpha(\lambda)$ continuous on $\left(\lambda_{1}, \lambda_{2}\right)$ and $|\alpha(\lambda)| \leqq M$,

$$
\begin{aligned}
\int_{a}^{b}\left[\frac{\partial}{\partial x} \int_{\lambda_{1}}^{\lambda_{2}} \alpha(\lambda) d_{\lambda} \int_{a}^{b} h(y) \frac{\partial}{\partial y} \Omega(x, y \mid \lambda) d y\right]^{2} d x & \leqq M^{2} \int_{a}^{b} h^{2}(x) d x=A . *
\end{aligned}
$$

The following interchanges of limits are justifiable for kernels of classes $H_{1}, I_{2}, \cdots, H_{m-1}$ :

* We assume this for kerncls of classes $H_{1}, H_{2}, \cdots, H_{m-1}$; for kernels $H_{1}$ this inequality follows by developments in (C), but is by no means obvious. 


$$
\begin{aligned}
\int_{\lambda_{1}}^{\lambda_{2}} \alpha(\lambda) d_{\lambda} & \int_{a}^{b} g(x)\left[\frac{\partial}{\partial x} \int_{a}^{b} h(y) \frac{\partial}{\partial y} \Omega(x, y \mid \lambda) d y\right] d x \\
& =\int_{a}^{b} g(x) \frac{\partial}{\partial x}\left[\int_{\lambda_{1}}^{\lambda_{2}} \alpha(\lambda) d_{\lambda} \int_{a}^{b} h(y) \frac{\partial}{\partial y} \Omega(x, y \mid \lambda) d y\right] d x \\
& =\int_{a}^{b} g(x) \frac{\partial}{\partial x}\left[\int_{a}^{b} \frac{\partial}{\partial y}\left[\int_{\lambda_{1}}^{\lambda_{2}} \alpha(\lambda) d_{\lambda} \Omega(x, y \mid \lambda)\right] h(y) d y\right] d x
\end{aligned}
$$

for $H_{1}$ this is assured in (C, p. 135).

The generalized Bessel's inequality for kernels of classes $H_{n}(n<m)$ is

$$
\int_{-\infty}^{\infty} d_{\lambda}\left[\int_{a}^{b} h(x)\left(\frac{\partial}{\partial x} \int_{a}^{b} h(y) \frac{\partial}{\partial y} \Omega(x, y \mid \lambda) d y\right) d x\right] \leqq \int_{a}^{b} h^{2}(x) d x
$$

(whenever $h(x) \subset L_{2}$ ).

Following the terminology of (C) one may call $\Omega(x, y \mid \lambda)$, corresponding to kernels $H_{n}$, closed in case (3.15) holds with the equality sign.

When $\Omega(x, y \mid \lambda)$ is closed then, for every $h(x) \subset L_{2}$,

$$
h(x)=\frac{d}{d x} \int_{-\infty}^{\infty} d_{\lambda}\left[\int_{a}^{b} h(y) \frac{\partial}{\partial y} \Omega(x, y \mid \lambda) d y\right]
$$

almost everywhere on $(a, b)$.

Suppose there is an operator $L$, as specified in Definition 2.3, associated with our kernel $K(x, y) \subset H_{n}$. Consider the equations

$$
\begin{array}{r}
L_{x}(\xi \mid \phi(x))-\lambda \int_{a}^{b} L_{x}\left(\xi \mid K^{\delta_{0}} \cdots, \delta_{n-1}(x, y)\right) \phi(y) d y=L_{x}(\xi \mid f(x)), \\
L_{x}(\xi \mid \phi(x))-\lambda \int_{a}^{b} L_{x}(\xi \mid K(x, y)) \phi(y) d y=L_{x}(\xi \mid f(x)),
\end{array}
$$

derived on the basis of (3.1) and (1.1), respectively. The following holds.

With $\Lambda=\beta \neq 0$ and $\phi^{\delta_{0}}, \cdots, \delta_{n-1}(x)$ denoting a solution of (3.1), the repeated limit, in the sense of weak convergence,

$$
\begin{aligned}
& \lim _{\delta_{0, r}} \lim _{\delta_{1, r}} \cdots \lim _{\delta_{n-1, r}} \phi^{\delta_{0}, r, \delta_{1}, r}, \cdots, \delta_{n-1, r}(x)=\phi(x) \quad \text { (suitable choice } \\
& \text { of } \left.\delta_{\nu, r}(>0 ; r=1,2, \cdots ; \nu=0, \cdots, n-1) ; \lim \delta_{r, r}=0\right)
\end{aligned}
$$

will exist and will constitute a solution of (3.18); moreover,

$$
\int_{a}^{b}\left|\phi^{2}(x)\right| d x \leqq M=\frac{|\lambda|^{2}}{\beta^{2}} \int_{a}^{b}|f(x)|^{2} d x .
$$


Corresponding to every function $\Omega(x, y \mid \lambda)$ defined as in (3.6a) the equation (3.18) has a solution

$$
\phi(x)=f(x)+\lambda \frac{\partial}{\partial x} \int_{-\infty}^{\infty} \frac{1}{\mu-\lambda} d_{\mu} \int_{a}^{b} f(y) \frac{\partial}{\partial y} \Omega(x, y \mid \mu) d y,
$$

provided $\Lambda \neq 0$; this solution satisfies the inequality (3.19a).

Suppose $h(y) \subset L_{2}$ and write (with $l>0$ )

$$
\begin{aligned}
\psi\left(x, l \mid \delta_{0}, \cdots, \delta_{n-1}\right) & \\
= & \left(\int_{l}^{\infty}+\int_{-\infty}^{-l}\right) \frac{1}{\mu} d_{\mu} \int_{a}^{b} \theta^{\delta_{0}}, \cdots, \delta_{n-1}(x, y \mid \mu) h(y) d y .
\end{aligned}
$$

With the $\delta_{i, r}[>0 ; r=1,2, \cdots ; i=0, \cdots, n-1]$ suitably chosen,

$$
\begin{aligned}
\psi\left(x, l \mid \delta_{0}, \cdots, \delta_{n-1, r}\right) & \rightarrow \psi\left(x, l \mid \delta_{0}, \cdots, \delta_{n-2}\right), \\
\psi\left(x, l \mid \delta_{0}, \cdots, \delta_{n-2, r}\right) & \rightarrow \psi\left(x, l \mid \delta_{0}, \cdots, \delta_{n-3}\right), \cdots, \\
\psi\left(x, l \mid \delta_{0, r}\right) & \rightarrow \psi(x, l) \quad(\text { as } r \rightarrow \infty),
\end{aligned}
$$

convergence being in the weak sense in $x$; moreover,

$$
\begin{gathered}
\int_{a}^{b} \psi^{2}(x, l) d x \leqq \frac{1}{l^{2}} \int_{a}^{b} h^{2}(x) d x, \\
\lim _{l \rightarrow \infty} L_{x}(\xi \mid \psi(x, l))=0 .
\end{gathered}
$$

For kernels $K(x, y)$ of classes $H_{n}(n<m)$ and $h(y) \subset L_{2}$

$$
\begin{aligned}
\int_{a}^{b} L_{x}(\xi \mid & K(x, y)) h(y) d y \\
= & \int_{-l}^{l} d_{\mu} \int_{a}^{b} L_{x}(\xi \mid K(x, s))\left[\frac{\partial}{\partial s} \int_{a}^{b} h(t) \frac{\partial}{\partial t} \Omega(s, t \mid \mu) d t\right] d s \\
& +L_{x}(\xi \mid \psi(x, l)) \\
= & \int_{-\infty}^{\infty} d_{\mu} \int_{a}^{b} L_{x}(\xi \mid K(x, s))\left[\frac{\partial}{\partial s} \int_{a}^{b} h(t) \frac{\partial}{\partial t} \Omega(s, t \mid \mu) d t\right] d s .
\end{aligned}
$$

On writing (with $l>0$ )

$$
w(x, l)=f(x)-\tau(x, l), \quad \tau(x, l)=\frac{\partial}{\partial x} \int_{-l}^{l} d_{\lambda} \int_{a}^{b} f(y) \frac{\partial}{\partial y} \Omega(x, y \mid \lambda) d y,
$$

we have ((3.23a) being a consequence of (3.13))

$$
\int_{a}^{b} \tau^{2}(x, l) d x \leqq \int_{a}^{b} f^{2}(y) d y=q,
$$




$$
\int_{a}^{b} w^{2}(x, l) d x \leqq 4 q
$$

and

$$
w\left(x, l_{v}\right) \rightarrow w(x), \quad \int_{a}^{b} w^{2}(x) d x \leqq 4 q \quad\left(l_{1}<l_{2}<\cdots\right),
$$

convergence being in the weak sense; moreover, $w(y)$ satisfies the equation

$$
\int_{a}^{b} L_{x}(\xi \mid K(x, y)) w(y) d y=0 \text {. }
$$

A consequence of the statements in connection with (3.23)-(3.23c) is the following. If the equation

$$
\int_{a}^{b} L_{x}(\xi \mid K(x, y)) \phi(y) d y=0
$$

has only the solution $\phi(\eta)=0$ (almost everywhere), then every $f(x) \subset L_{2}$ has the representation

(3.24a) $\quad f(x)=\frac{d}{d x} \int_{-\infty}^{\infty} d_{\lambda} \int_{a}^{b} f(y) \frac{\partial}{\partial y} \Omega(x, y \mid \lambda) d y \quad$ (almost everywhere).

Let $\Delta q(\lambda)=q\left(\lambda^{\prime}\right)-q\left(\lambda^{\prime \prime}\right)$ (real $\left.\lambda^{\prime}, \lambda^{\prime \prime}, \lambda^{\prime}<\lambda^{\prime \prime}\right)$; then for all kernels of classes $H_{n}(n<m)$ and for all $h(y) \subset L_{2}$

$$
\begin{aligned}
& L_{x}\left(\xi \mid \frac{\partial}{\partial x} \Delta \int_{a}^{b} h(y) \frac{\partial}{\partial y} \Omega(x, y \mid \lambda) d y\right) \\
& \quad-\int_{a}^{b} L_{x}(\xi \mid K(x, s))\left[\frac{\partial}{\partial s} \int_{\lambda^{\prime}}^{\lambda^{\prime \prime}} \mu d_{\mu} \int_{a}^{b} h(y) \frac{\partial}{\partial y} \Omega(s, y \mid \mu) d y\right] d s=0 ;
\end{aligned}
$$

in particular,

$$
\begin{aligned}
L_{x}\left(\xi \mid \frac{\partial}{\partial x} \Delta \Omega(x, y \mid \lambda)\right) & \\
& -\int_{a}^{b} L_{x}(\xi \mid K(x, s))\left[\frac{\partial}{\partial s} \int_{\lambda^{\prime}}^{\lambda^{\prime \prime}} \mu d_{\mu} \Omega(s, y \mid \mu)\right] d s=0 .
\end{aligned}
$$

Also for $K(x, y) \subset H_{n}(n<m)$

$$
\begin{aligned}
\int_{a}^{b} L_{x}(\xi \mid K(x, y)) h(y) d y & \\
& =\int_{-\infty}^{\infty} \frac{1}{\mu} d_{\mu} L_{x}\left(\xi \mid \frac{\partial}{\partial x} \int_{a}^{b} h(y) \frac{\partial}{\partial y} \Omega(x, y \mid \mu) d y\right)
\end{aligned}
$$


for all $h(y) \subset L_{2}$. Furthermore the following relation will hold, for $K(x, y) \subset H_{n}(n<m)$,

$$
\begin{aligned}
L_{x}\left(\xi \mid \frac{\partial}{\partial x} \int_{\lambda^{\prime}}^{\lambda^{\prime \prime}} \mu d_{\mu} \Omega(x, y \mid \mu)\right) & \\
& -\lambda \int_{a}^{b} L_{x}(\xi \mid K(x, s))\left[\frac{\partial}{\partial s} \int_{\lambda^{\prime}}^{\lambda^{\prime \prime}} \mu d_{\mu} \Omega(s, y \mid \mu)\right] d s \\
& =L_{x}\left(\xi \mid \frac{\partial}{\partial x} \int_{\lambda^{\prime}}^{\lambda^{\prime \prime}}(\mu-\lambda) d_{\mu} \Omega(x, y \mid \mu)\right) \quad(\text { if } \lambda=0) .
\end{aligned}
$$

4. Developments without the aid of operators $L$. In $\S 3$ we have assumed and have stated certain facts (refer to the text from (3.4) to (3.27))for classes $H_{n}(n=1,2, \cdots, m-1)$; an examination of Carleman's work leads to the conclusion that these statements certainly hold for Carleman's kernels $H_{1}$. We shall now prove that the results asserted from (3.4) to (3.28) hold for kernels $K(x, y) \subset H_{m}$, as well. This will establish the theory for kernels included in $H_{v}$, where $\nu(>0)$ is any finite integer.

Let

$$
K_{1}(x, y) \subset H_{m},
$$

the spectrum corresponding to $K_{1}^{\delta_{0}}, \cdots, \delta_{m-1}(x, y)$ being the function defined in (3.3), (3.3a), (3.3b), with $n=m$. In the definition of the spectrum are involved numbers $\lambda_{\nu}{ }^{\delta_{0}}, \cdots, \delta_{m-1}$ and functions $\phi_{\nu}{ }_{\nu}, \cdots, \delta_{m-1}(x)$ (orthogonal normal set), satisfying equation (3.3c), where we now write

$$
n=m, \quad K^{\delta_{0}}, \cdots, \delta_{n-1}(x, y)=K_{1}^{\delta_{0}} \cdots, \delta_{m-1}(x, y) .
$$

If one forms the function

$$
\Omega_{1}^{\delta_{0}}, \cdots, \delta_{m-1}(x, y \mid \lambda)=\int_{a}^{x} \int_{a}^{y} \theta_{1}^{\delta_{0}}, \cdots, \delta_{m-1}(x, y \mid \lambda) d x d y
$$

(cf. (3.4)) and notes that this function is a $\Omega(x, y \mid \lambda)$ belonging to $H_{0}$, it is observed that in consequence of (3.5)-(3.6), there exist limits

$$
\begin{aligned}
& \lim _{\delta_{m-1, r}} \Omega_{1} \delta_{0}, \cdots, \delta_{m-1, r}(x, y \mid \lambda)=\Omega_{1}^{\delta_{0}} \cdots, \delta_{m-2}(x, y \mid \lambda), \\
& \lim _{\delta_{m-2, r}} \Omega_{1} \delta_{0}, \cdots, \delta_{m-2, r}(x, y \mid \lambda)=\Omega_{1}^{\delta_{0}, \cdots, \delta_{m-3}}(x, y \mid \lambda), \cdots, \\
& \lim _{\delta_{1, r}} \Omega_{1}^{\delta_{0}, \delta_{1, r}}(x, y \mid \lambda)=\Omega_{1}^{\delta_{0}}(x, y \mid \lambda) \\
& {\left[\lim _{r} \delta_{i, r}=0 ; i=m-1, m-2, \cdots, 1\right] .}
\end{aligned}
$$


The latter limit is a $\Omega(x, y \mid \lambda)$-function belonging to the class $H_{m-1}$. This function, accordingly, satisfies (3.7), (3.7a). Whence the "Compactness Theorem" (\$2) can be applied, thus enabling one to assert that

$$
\lim _{\delta_{0 . r}} \Omega_{1}^{\delta_{0, r}}(x, y \mid \lambda)=\Omega_{1}(x, y \mid \lambda) \quad \text { (suitable } \delta_{0, r} ; r=1,2, \cdots \text { ) }
$$

exists, with the limiting function satisfying (3.7), (3.7a). We note that $\Omega_{1}(x, y \mid \lambda)$ is a $\Omega(x, y \mid \lambda)$-function belonging to our kernel (4.1) and that it may be discontinuous in $\lambda$ for, say, $\lambda_{1}{ }^{\prime}, \lambda_{2}{ }^{\prime}, \cdots$.

We supposed that (3.8) holds for $\Omega$-functions belonging to $H_{m-1}$; thus

$$
\int_{a}^{b}\left|\frac{\partial}{\partial y} \Omega_{1}^{\delta_{0}}(x, y \mid \lambda)\right|^{2} d y \leqq x-a ;
$$

by Theorem 1.3 and in view of (4.3)

$$
\frac{\partial}{\partial y} \Omega_{1} \delta_{0, r}(x, y \mid \lambda) \rightarrow \frac{\partial}{\partial y} \Omega_{1}(x, y \mid \lambda) \quad\left(\text { as } r \rightarrow \infty \text {; suitable } \delta_{0, r}>0\right),
$$

convergence being in the weak sense in $y$; moreover,

$$
\int_{a}^{b}\left|\frac{\partial}{\partial y} \Omega_{1}(x, y \mid \lambda)\right|^{2} d y \leqq x-a .
$$

These considerations enable us to assert (3.8), (3.8a) for the class $H_{m}$.

By (3.9), stated for $\Omega_{1}^{\delta_{0}}(x, y \mid \lambda)$, with the aid of Theorem 1.3 we obtain the relation

$$
\lim _{r} \frac{\partial}{\partial x} \int_{y=a}^{b} h(y) \frac{\partial}{\partial y} \Omega_{1}^{\delta_{0}, r}(x, y \mid \lambda) d y=\frac{\partial}{\partial x} \int_{y=a}^{b} h(y) \frac{\partial}{\partial y} \Omega_{1}(x, y \mid \lambda) d y
$$

[weak convergence in $x$; suitable $\delta_{0, r}(r=1,2, \cdots)$ ];

moreover, (3.9) will hold for $\Omega_{1}(x, y \mid \lambda)$.

With the aid of Theorem 1.4, in view of (4.5) and on writing

$$
f_{r}(x)=\frac{\partial}{\partial x} \int_{a}^{b} h(y) \frac{\partial}{\partial y} \Omega_{1}^{\delta_{0}, r}(x, y \mid \lambda) d y,
$$

we get (whenever $g(x), h(x) \subset L_{2}$ )

$$
\begin{aligned}
\lim \int_{a}^{b} g(x)\left[\frac{\partial}{\partial x} \int_{a}^{b} h(y)\right. & \left.\frac{\partial}{\partial y} \Omega_{1}^{\delta_{0}, r}(x, y \mid \lambda) d y\right] d x \\
& =\int_{a}^{b} g(x)\left[\frac{\partial}{\partial x} \int_{a}^{b} h(y) \frac{\partial}{\partial y} \Omega_{1}(x, y \mid \lambda) d y\right] d x
\end{aligned}
$$

which is (3.10) for $\Omega(x, y \mid \lambda)$. 
Formula (3.11a) will hold in particular for $\Omega_{1}^{\delta_{0}, r}(x, y \mid \lambda)$; on taking account of (4.6) it is concluded that (3.11a) holds in the limit, that is with $\Omega(x, y \mid \lambda)$ replaced by $\Omega_{1}(x, y \mid \lambda)$. The inequality thus obtained enables us to assert that (3.11) will hold also for $\Omega_{1}(x, y \mid \lambda)$.

By (4.6), (3.11a), with $\Omega=\Omega_{1}^{\delta_{0}, r}$, in virtue of Theorem 1.1 it is deduced that

$$
\begin{aligned}
\lim _{r} \int_{\lambda_{1}}^{\lambda_{2}} \alpha(\lambda) d_{\lambda} & \int_{a}^{b} g(x)\left[\frac{\partial}{\partial x} \int_{a}^{b} h(y) \frac{\partial}{\partial y} \Omega_{1}^{\delta_{0}, r}(x, y \mid \lambda) d y\right] d x \\
& =\int_{\lambda_{1}}^{\lambda_{2}} \alpha(\lambda) d_{\lambda} \int_{a}^{b} g(x)\left[\frac{\partial}{\partial x} \int_{a}^{b} h(y) \frac{\partial}{\partial y} \Omega_{1}(x, y \mid \lambda) d y\right] d x
\end{aligned}
$$

(whenever $\alpha(\lambda)$ is continuous and $g(x), h(y) \subset L_{2}$ ); that is, (3.12) holds for $\Omega_{1}(x, y \mid \lambda)$.

In (4.7) replace $x$ by $t$ and let

$$
g(t)=1 \quad(a \leqq t \leqq x), \quad g(t)=0 \quad(x<t \leqq b) ;
$$

then it is deduced that

$$
\begin{aligned}
\lim _{r} \int_{\lambda_{1}}^{\lambda_{2}} \alpha(\lambda) d_{\lambda} \int_{a}^{b} h(y) \frac{\partial}{\partial y} \Omega_{1}^{\delta_{0}, r}(x, y \mid \lambda) d y & \\
= & \int_{\lambda_{1}}^{\lambda_{2}} \alpha(\lambda) d_{\lambda} \int_{a}^{b} h(y) \frac{\partial}{\partial y} \Omega_{1}(x, y \mid \lambda) d y \\
& \left(\alpha(\lambda) \text { continuous, } h(y) \subset L_{2}\right) ;
\end{aligned}
$$

the relationship (4.9) will hold also for $\Omega$-functions belonging to classes $H$, $(\nu<m)$. Now, we may write (3.13) for $\Omega_{1}^{\delta_{0}, r}(x, y \mid \lambda)$; the inequality so obtained, together with Theorem 1.3, would imply that, if the $\delta_{0, r}$ are suitably chosen,

$$
\frac{\partial}{\partial x} \int_{\lambda_{1}}^{\lambda_{2}} \alpha(\lambda) d_{\lambda} \int_{a}^{b} h(y) \frac{\partial}{\partial y} \Omega_{1}^{\delta_{0}, r}(x, y \mid \lambda) d y \rightarrow \Gamma(x, \lambda) \quad(\text { as } r \rightarrow \infty),
$$

convergence being in the weak sense (in $x$ ); by (4.9)

$$
\Gamma(x, \lambda)=\frac{\partial}{\partial x} \int_{\lambda_{1}}^{\lambda_{2}} \alpha(\lambda) d_{\lambda} \int_{a}^{b} h(y) \frac{\partial}{\partial y} \Omega_{1}(x, y \mid \lambda) d y,
$$

and (cf. (3.13)), in accordance with Theorem 1.3,

$$
\int|\Gamma(x, \lambda)|^{2} d x \leqq A .
$$

Thus it is concluded that (3.13) holds for the class $H_{m}$; it is also clear that (4.9a), (4.9b) hold for all classes $H_{\nu}(\nu \leqq m)$. 
We now proceed to establish the first equality (3.14) for $\Omega_{1}$. With $\Omega_{1}{ }^{\delta_{0}, r}$ belonging to $H_{m-1}$, this equality takes the form

$$
\begin{aligned}
\int_{\lambda_{1}}^{\lambda_{2}} \alpha(\lambda) & d_{\lambda} \int_{a}^{b} g(x)\left[\frac{\partial}{\partial x} \int_{a}^{b} h(y) \frac{\partial}{\partial y} \Omega_{1}^{\delta_{0}, r}(x, y \mid \lambda) d y\right] d x \\
= & \int_{a}^{b} g(x) \frac{\partial}{\partial x}\left[\int_{\lambda_{1}}^{\lambda_{2}} \alpha(\lambda) d_{\lambda} \int_{a}^{b} h(y) \frac{\partial}{\partial y} \Omega_{1}^{\delta_{0}, r}(x, y \mid \lambda) d y\right] d x
\end{aligned}
$$

In view of (3.13) (for $\Omega_{1}^{\delta_{0}, r}$ ), (4.9a), and (4.9b), application of Theorem 1.4 will yield the result

$$
\begin{aligned}
\lim _{r} \int_{a}^{b} g(x) & \frac{\partial}{\partial x}\left[\int_{\lambda_{1}}^{\lambda_{2}} \alpha(\lambda) d_{\lambda} \int_{a}^{b} h(y) \frac{\partial}{\partial y} \Omega_{1}^{\delta_{0}, r}(x, y \mid \lambda) d y\right] d x \\
& =\int_{a}^{b} g(x) \frac{\partial}{\partial x}\left[\int_{\lambda_{1}}^{\lambda_{2}} \alpha(\lambda) d_{\lambda} \int_{a}^{b} h(y) \frac{\partial}{\partial y} \Omega_{1}(x, y \mid \lambda) d y\right] d x
\end{aligned}
$$

We shall have [whenever $g(x) \subset L_{2} ;$ suitable $\delta_{0, r}(r=1,2, \cdots)$ ].

$$
\begin{aligned}
\lim _{r} \int_{\lambda_{1}}^{\lambda_{2}} \alpha(\lambda) d_{\lambda} & \int_{a}^{b} g(x)\left[\frac{\partial}{\partial x} \int_{a}^{b} h(y) \frac{\partial}{\partial y} \Omega_{1}^{\delta_{0}, r}(x, y \mid \lambda) d y\right] d x \\
& =\int_{\lambda_{1}}^{\lambda_{2}} \alpha(\lambda) d_{\lambda} \int_{a}^{b} g(x)\left[\frac{\partial}{\partial x} \int_{a}^{b} h(y) \frac{\partial}{\partial y} \Omega_{1}(x, y \mid \lambda) d y\right] d x,
\end{aligned}
$$

if it is shown that

$$
\begin{aligned}
\lim _{r} \int_{a}^{b} g(x)\left[\frac{\partial}{\partial x} \int_{a}^{b} h(y)\right. & \left.\frac{\partial}{\partial y} \Omega_{1}^{\delta_{0}, r}(x, y \mid \lambda) d y\right] d x \\
& =\int_{a}^{b} g(x)\left[\frac{\partial}{\partial x} \int_{a}^{b} h(y) \frac{\partial}{\partial y} \Omega_{1}(x, y \mid \lambda) d y\right] d x
\end{aligned}
$$

and that

$$
\text { Var. } \int_{a}^{b} g(x)\left[\frac{\partial}{\partial x} \int_{a}^{b} h(y) \frac{\partial}{\partial y} \Omega_{1}^{\delta_{0}, r}(x, y \mid \lambda) d y\right] d x \leqq B,
$$

where $B$ is independent of $\delta_{0, r}$; this it is possible to assert in consequence of Theorem 1.1. Now, (1) holds in view of (3.10) (with $\Omega=\Omega_{1}$ ); on the other hand, (2) is implied by (3.11a) (inasmuch as $\Omega_{1}^{\delta_{0}, r}$ is of class $H_{m-1}$ ). Thus $(4.10 \mathrm{~b})$ is seen to be true; together with $(4.10 \mathrm{a})$ this relation enables us to deduce from (4.10) that the first equality of (3.14) holds for $\Omega=\Omega_{1}$.

In view of (4.10a) the second equality (3.14) will be established for $\Omega=\Omega_{1}$, provided it is shown that 


$$
\begin{aligned}
& \lim _{r} \int_{a}^{b} g(x) \frac{\partial}{\partial x}\left[\int_{a}^{b} \frac{\partial}{\partial y}\left[\int_{\lambda_{1}}^{\lambda_{2}} \alpha(\lambda) d_{\lambda} \Omega_{1}^{\delta_{0}, r}(x, y \mid \lambda)\right] h(y) d y\right] d x \\
&=\int_{a}^{b} g(x) \frac{\partial}{\partial x}\left[\int_{a}^{b} \frac{\partial}{\partial y}\left[\int_{\lambda_{1}}^{\lambda_{2}} \alpha(\lambda) d_{\lambda} \Omega_{1}(x, y \mid \lambda)\right] h(y) d y\right] d x .
\end{aligned}
$$

If one equates the first and the last member of (3.14), writing

$$
\Omega=\Omega_{1}^{\delta_{0, r},} \quad g=1 \quad(\text { on }(a, x)), \quad g=0 \quad(\text { on }(x, b)),
$$

it is deduced that

$$
\begin{aligned}
\int_{\lambda_{1}}^{\lambda_{2}} \alpha(\lambda) d_{\lambda} \int_{a}^{b} h(y) \frac{\partial}{\partial y} \Omega_{1}^{\delta_{0}, r}(x, y \mid \lambda) d y \\
\quad=\int_{a}^{b} \frac{\partial}{\partial y}\left[\int_{\lambda_{1}}^{\lambda_{2}} \alpha(\lambda) d_{\lambda} \Omega_{1}^{\delta_{0}, r}(x, y \mid \lambda)\right] h(y) d y ;
\end{aligned}
$$

by (3.13) (with $\Omega=\Omega^{\delta_{0}, r}$ ) the latter equality implies that

$$
\int_{a}^{b}\left[\frac{\partial}{\partial x} \int_{a}^{b} \frac{\partial}{\partial y}\left[\int_{\lambda_{1}}^{\lambda_{2}} \alpha(\lambda) d_{\lambda} \Omega_{1} \delta_{0, r}(x, y \mid \lambda)\right] h(y) d y\right]^{2} d x \leqq A .
$$

In view of (4.10d) and in consequence of Theorems 1.4, 1.3 it is observed that (3) and hence the second equality (3.14) will hold for $\Omega_{1}$, provided that

$$
\begin{aligned}
\lim _{r} \int_{a}^{b} \frac{\partial}{\partial y} & {\left[\int_{\lambda_{1}}^{\lambda_{2}} \alpha(\lambda) d_{\lambda} \Omega_{1} \delta_{0, r}(x, y \mid \lambda)\right] h(y) d y } \\
& =\int_{a}^{b} \frac{\partial}{\partial y}\left[\int_{\lambda_{1}}^{\lambda_{2}} \alpha(\lambda) d_{\lambda} \Omega_{1}(x, y \mid \lambda)\right] h(y) d y \quad \text { (suitable } \delta_{0, r} \text { ). }
\end{aligned}
$$

In virtue of (4.10c) it is concluded that (4) will hold if

$$
\begin{aligned}
\lim _{r} \int_{\lambda_{1}}^{\lambda_{2}} \alpha(\lambda) d_{\lambda} \int_{a}^{b} h(y) \frac{\partial}{\partial y} \Omega_{1}^{\delta_{0, r}}(x, y \mid \lambda) d y \\
=\int_{a}^{b} \frac{\partial}{\partial y}\left[\int_{\lambda_{1}}^{\lambda_{2}} \alpha(\lambda) d_{\lambda} \Omega_{1}(x, y \mid \lambda)\right] h(y) d y ;
\end{aligned}
$$

that is, in view of (4.9), if

$$
\begin{aligned}
\int_{\lambda_{1}}^{\lambda_{2}} \alpha(\lambda) d_{\lambda} \int_{a}^{b} h(y) \frac{\partial}{\partial y} \Omega_{1}(x, y \mid \lambda) d y \\
=\int_{a}^{b} \frac{\partial}{\partial y}\left[\int_{\lambda_{1}}^{\lambda_{2}} \alpha(\lambda) d_{\lambda} \Omega_{1}(x, y \mid \lambda)\right] h(y) d y .
\end{aligned}
$$


Now, (4.10e) can be established with the aid of (4.10c). In fact, by (4.9) the first member of $(4.10 \mathrm{c})$ will yield in the limit the first member of $(4.10 \mathrm{e})$; on the other hand, for suitable $\delta_{0, r}(r=1,2, \cdots)$

(4.11) $\lim _{r} \int_{a}^{b} h(y)\left[\frac{\partial}{\partial y} \int_{\lambda_{1}}^{\lambda_{2}} \alpha(\lambda) d_{\lambda} \Omega_{1}{ }^{\delta_{0}, r}(x, y \mid \lambda)\right] d y=$ second member of

because, by (3.13) (with $\Omega=\Omega_{1}^{\delta_{0}, r}$ and $h=1$ on $(a, y)$ and $h=0$ on $(y, b)$ ),

$$
\int_{a}^{b}\left[\frac{\partial}{\partial x} \int_{\lambda_{1}}^{\lambda_{2}} \alpha(\lambda) d_{\lambda} \Omega_{1} \delta_{0}, r(x, y \mid \lambda)\right]^{2} d x \leqq A(r=1,2, \cdots), *
$$

and since

$$
\lim _{r} \int_{\lambda_{1}}^{\lambda_{2}} \alpha(\lambda) d_{\lambda} \Omega_{1}^{\delta_{0}, r}(x, y \mid \lambda)=\int_{\lambda_{1}}^{\lambda_{2}} \alpha(\lambda) d_{\lambda} \Omega_{1}(x, y \mid \lambda) . \dagger
$$

To ascertain the truth of (4.11) on the basis of (4.11a) and (4.11b) one needs only to take note of Theorem 1.3 and of Theorem 1.4. With (4.10e) established we have (4) secured, as well as the second equality (3.14) (for $\Omega_{1}$ ).

Thus, (3.14) holds for the class $H_{m}$.

To establish the generalized Bessel's inequality for $\Omega_{1}$ it is observed first that, in consequence of (3.11a) with

$$
\Omega=\Omega_{1}^{\delta_{0, r},}, \quad g(x)=h(x) \subset L_{2},
$$

it follows that

$$
\text { Var. } \int_{a}^{b} h(x)\left[\frac{\partial}{\partial x} \int_{a}^{b} h(y) \frac{\partial}{\partial y} \Omega_{1}^{\delta_{0}, r}(x, y \mid \lambda) d y\right] d x \leqq \int_{a}^{b} h^{2}(x) d x .
$$

Also

$$
\begin{aligned}
\lim _{r} \int_{a}^{b} h(x)\left[\frac{\partial}{\partial x} \int_{a}^{b} h\right. & \left.(y) \frac{\partial}{\partial y} \Omega_{1}^{\delta_{0}, r}(x, y \mid \lambda) d y\right] d x \\
& =\int_{a}^{b} h(x)\left[\frac{\partial}{\partial x} \int_{a}^{b} h(y) \frac{\partial}{\partial y} \Omega_{1}(x, y \mid \lambda) d y\right] d x
\end{aligned}
$$

which is deduced from (3.10) (for $\Omega=\Omega_{1}$ and $g(x)=h(x)$ ). In view of (4.12) and (4.12a), with the aid of Theorem 1.1, and on writing (3.15) (with $\left.\Omega=\Omega_{1}{ }^{\delta_{0}, r}\right)$, and on letting $r \rightarrow \infty$, it is inferred without difficulty that

* Here $x$ and $y$ may be interchanged.

$\dagger$ This relation is a consequence of the inequality Var. $\Omega_{1}{ }^{\delta_{0, r}} \leqq[(x-a)(y-a)]^{1 / 2}$ and of the Theorem 1.1 ; (4.11b) also follows (4.9). 


$$
\int_{-\infty}^{\infty} d_{\lambda}\left[\int_{a}^{b} h(x)\left[\frac{\partial}{\partial x} \int_{a}^{b} h(y) \frac{\partial}{\partial y} \Omega_{1}(x, y \mid \lambda) d y\right] d x\right] \leqq \int_{a}^{b} h^{2}(x) d x,
$$

which is the desired inequality.

When $\Omega_{1}$ is closed, so that (4.13) holds with the equality sign, we obtain the representation (3.16), with $\Omega=\Omega_{1}$, by a device of the type employed in (C). That is, replace $h(x)$ in (4.13) by $h(x)+g(x)$, obtaining

$$
\begin{aligned}
2 \int_{a}^{b} h(x) g(x) d x & =\int_{-\infty}^{\infty} d_{\lambda}\left[\int_{a}^{b} h(x)\left[\frac{\partial}{\partial x} \int_{a}^{b} g(y) \frac{\partial}{\partial y} \Omega_{1}(x, y \mid \lambda) d y\right] d x\right] \\
& +\int_{-\infty}^{\infty} d_{\lambda}\left[\int_{a}^{b} g(x)\left[\frac{\partial}{\partial x} \int_{a}^{b} h(y) \frac{\partial}{\partial y} \Omega_{1}(x, y \mid \lambda) d y\right] d x\right] .
\end{aligned}
$$

In the first term of the second member of (4.14) interchange $x$ and $y$ and then let

$$
g=1 \quad(\text { on }(a, x)), \quad g=0 \quad(\text { on }(x, b)) ;
$$

the representation (3.16) (with $\Omega=\Omega_{1}$ ) will result immediately.

Thus, all the statements which have been made in $\$ 3$ up to (3.16), inclusive, hold for the class $H_{m}$, as well. The statements of $\S 3$, just referred to, have been made for classes $H_{n}(n<m)$ without the use of operators $L$ (Definition 2.3). The results therein indicated have been extended in the above to the class $H_{m}$; in the process of the extension operators $L$ have not been employed. Hence the induction is complete with respect to the statements, in question, of $\S 3$. We state this result as follows.

Theorem 4.1. With classes $H_{n}$ specified by Definition 2.2, all the statements made in $\$ 3$ up to (3.16) (inclusive) will hold true for all classes $H_{n}(n=1,2, \cdots)$.

5 . Developments on the basis of operators $L$. Let $L^{\prime}$ be an operator as specified in Definition 2.3 and supposed to exist, associated with our kernel $K_{1}(x, y) \subset H_{m}$. We form the equation

$$
L_{x}^{\prime}\left(\xi \mid \phi_{1}(x)\right)-\lambda \int_{a}^{b} L_{x}^{\prime}\left(\xi \mid K_{1}(x, y)\right) \phi_{1}(y) d y=L_{x}^{\prime}(\xi \mid f(x))
$$

(with given $f(x) \subset L_{2}$ ), as well as the related equation

$$
L_{x}^{\prime}\left(\xi \mid \phi_{1}^{\delta_{0}, r}(x)\right)-\lambda \int_{a}^{b} L_{x}^{\prime}\left(\xi \mid K_{1}^{\delta_{0}, r}(x, y)\right) \phi_{1}^{\delta_{0}, r}(y) d y=L_{x}^{\prime}(\xi \mid f(x)) .
$$

It is essential to demonstrate that the operator $L$ ' is "associated" (in the sense of Definition 2.3) with the kernel $K_{1}^{\delta_{0}, r}(x, y) \subset H_{m-1}$. Thus the following relations are to be verified, with $K^{1}=K_{1}^{\delta_{0}}$ : 


$$
L_{x}^{\prime}\left(\xi \mid K^{1}(x, y)\right) \subset L_{2}
$$

(2)

$$
\begin{aligned}
& \mid L_{x}^{\prime}\left(\xi\left|K^{1 \delta_{1}, \cdots, \delta_{m-1}}(x, y)\right|<\gamma(\xi \mid y) \subset L_{2}\right. \\
& L_{x}^{\prime}\left(\xi \mid K^{1 \delta_{1}}, \cdots, \delta_{m-1}(x, y)\right) \underset{\left(\delta_{m-1}\right)}{\longrightarrow} L_{x}^{\prime}\left(\xi \mid K^{1 \delta_{1}, \cdots, \delta_{m-2}}(x, y)\right) \\
& \underset{\left(\delta_{m-2}\right)}{\longrightarrow} L_{x}^{\prime}\left(\xi \mid K^{1 \delta_{1}, \cdots, \delta_{m-3}}(x, y)\right) \\
& \longrightarrow \cdots L_{x}^{\prime}\left(\xi \mid K^{1 \delta_{1}}\right) \underset{\left(\delta_{1}\right)}{\longrightarrow} L_{x}^{\prime}\left(\xi \mid K^{1}(x, y)\right) ;
\end{aligned}
$$

$$
\begin{aligned}
& \text { (4) } L_{x}^{\prime}\left(\xi \mid f_{\nu}(x)\right) \underset{(\nu)}{\longrightarrow} L_{x}^{\prime}(\xi \mid f(x)), \quad \text { if } f_{\nu}(x) \rightarrow f(x) \text { in the weak sense; } \\
& \text { (5) } \int_{a}^{b} L_{x}^{\prime}\left(\xi \mid K^{1 \delta_{1}, \cdots, \delta_{m-1}}(x, y)\right) \phi(y) d y=L_{x}^{\prime}\left(\xi \mid \int_{a}^{b} K^{1 \delta_{1}}, \cdots, \delta_{m-1}(x, y) \phi(y) d y\right)
\end{aligned}
$$

$$
\text { (whenever } \phi(y) \subset L_{2} \text { ). }
$$

If we designate by $\left(2.20^{\prime}\right)-\left(2.24^{\prime}\right)$ the conditions (2.20)-(2.24), with $K, L$ and $n$ replaced by $K_{1}, L^{\prime}$, and $m$, the truth of (1)-(5) is inferred as follows. Conditions (2), (4), (5) are precisely the conditions $\left(2.21^{\prime}\right),\left(2.23^{\prime}\right),\left(2.24^{\prime}\right)$. The relations (3) are identical with those of $\left(2.22^{\prime}\right)$, the last limiting relation in (2.22') being omitted. As to (1), it is observed that, in view of (2) and (3),

$$
\left|L_{x}^{\prime}\left(\xi \mid K^{1}(x, y)\right)\right| \leqq \gamma(\xi \mid y) \subset L_{2}
$$

which, together with other considerations, establishes (1).

By (3.19) and (3.19a) the equation (5.2) has a solution ${ }^{*} \phi_{1}{ }^{\delta_{0}, \eta}(x)$, such that

$$
\int_{a}^{b}\left|\phi_{1} \delta_{0, r}(x)\right|^{2} d x \leqq \frac{|\lambda|^{2}}{\beta^{2}} \int_{a}^{b}|f(x)|^{2} d x=M \quad(\text { if } \lambda=\beta \neq 0) .
$$

In consequence of Theorem 1.3, applicable in view of (5.3), we have for suitable $\delta_{0, r}\left(>0 ; r=1,2, \cdots ; \lim _{r} \delta_{0, r}=0\right)$

$$
\lim _{r} \phi_{1}^{\delta_{0}, r}(x)=\phi_{1}(x) \text {, }
$$

convergence being in the weak sense; moreover,

$$
\int_{a}^{b}\left|\phi_{1}(x)\right|^{2} d x \leqq M
$$

It remains to demonstrate that $\phi_{1}(x)$ is a solution of (5.1). Substitute the function $\phi_{1}^{\delta_{0}, r}(x)$ (referred to in (5.3)) in (5.2) and let $r \rightarrow \infty$. We shall have

$$
\lim _{r} L_{x}^{\prime}\left(\xi \mid \phi_{1}^{\delta_{0}, r}(x)\right)=L_{x}^{\prime}\left(\xi \mid \phi_{1}(x)\right)
$$

* This solution is obtained as a repeated limit, according to (3.19). 
by (5.4) and $\left(2.23^{\prime}\right)$. On the other hand,

(5.5a) $\lim _{r} \int_{a}^{b} L_{x}^{\prime}\left(\xi \mid K_{1}^{\delta_{0}, r}(x, y)\right) \phi_{1}^{\delta_{0}, r}(y) d y=\int_{a}^{b} L_{x}^{\prime}\left(\xi \mid K_{1}(x, y)\right) \phi_{1}(y) d y$.

This is a consequence of (5.3), (5.4), of the last limiting relation $\left(2.22^{\prime}\right)$, and of the inequality

$$
\left|L_{x}^{\prime}\left(\xi \mid K_{1}^{\delta_{0}, r}(x, y)\right)\right| \leqq \gamma(\xi \mid y) \subset L_{2}
$$

in fact, these conditions enable application of Theorem 1.5. In virtue of (5.5) and (5.5a) it can be asserted that the function $\phi_{1}(x)$, defined in (5.4) constitutes $a$ solution of (5.1) (for $\beta \neq 0$ ). In view of the definition of $\phi_{1}{ }^{\delta_{0}, r}(x)$ and in consequence of (5.4) it is observed that $\phi_{1}(x)$ is a repeated limit. The statement in connection with (3.19), (3.19a) can thus be made for the class $H_{m}$.

The important formula (3.20) will be extended to our equation (5.1) with the aid of the following relation

$$
\begin{aligned}
\lim _{r} \int_{-\infty}^{\infty} \frac{1}{\mu-\lambda} d_{\mu} \int_{a}^{b} f(y) \frac{\partial}{\partial y} \Omega_{1}^{\delta_{0}, r}(x, y \mid \mu) d y \\
\quad=\int_{-\infty}^{\infty} \frac{1}{\mu-\lambda} d_{\mu} \int_{a}^{b} f(y) \frac{\partial}{\partial y} \Omega_{1}(x, y \mid \mu) d y,
\end{aligned}
$$

which we shall now proceed to prove. Let us write (with $l>\mid$ real part of $\lambda \mid$ )

$$
\begin{aligned}
\int_{-\infty}^{\infty} \frac{1}{\mu-\lambda} d_{\mu} p_{r}(x, \mu) & =\int_{-l}^{l} \frac{1}{\mu-\lambda} d_{\mu} p_{r}(x, \mu)+R_{l, r}(x, \lambda), \\
R_{l, r}(x, \lambda) & =\left(\int_{l}^{\infty}+\int_{-\infty}^{-l}\right) \frac{1}{\mu-\lambda} d_{\mu} p_{r}(x, \mu), \\
p_{r}(x, \mu) & =\int_{a}^{b} f(y) \frac{\partial}{\partial y} \Omega_{1}^{\delta_{0}, r}(x, y \mid \mu) d y, \\
R_{l}(x, \lambda) & =\left(\int_{l}^{\infty}+\int_{-\infty}^{-l}\right) \frac{1}{\mu-\lambda} d_{\mu} \int_{a}^{b} f(y) \frac{\partial}{d y} \Omega_{1}(x, y \mid \mu) d y .
\end{aligned}
$$

By (3.11a), with

$$
\left.\left.\Omega=\Omega_{1}^{\delta_{0, r},} \quad h(y)=f(y), \quad g=1 \text { (on }(a, x)\right), \quad g=0 \text { (on }(x, b)\right),
$$

we have

$$
\text { Var. } p_{r}(x, \mu) \leqq\left[\int_{a}^{b} f^{2}(x) d x\right]^{1 / 2}(x-a)^{1 / 2} \leqq A .
$$

* This is the relation obtained immediately preceding (5.3). 
On the other hand, because of (3.9a) (with $\Omega=\Omega_{1}$ )

$$
\lim _{r} p_{r}(x, \mu)=\int_{a}^{b} f(y) \frac{\partial}{\partial y} \Omega_{1}(x, y \mid \mu) d y=p(x, \mu) .
$$

In view of $(5.7 \mathrm{a})$

$$
\text { Var. } p(x, \mu) \leqq A .
$$

In consequence of (5.7a) and (5.7b), application of Theorem 1.1 will yield the result

$$
\lim _{r} \int_{-l}^{l} \frac{1}{\mu-\lambda} d_{\mu} p_{r}(x, \mu)=\int_{-l}^{l} \frac{1}{\mu-\lambda} d_{\mu} p(x, \mu) .
$$

It is also noted that, by (5.7), (5.7a) and (5.7c),

$$
\left|R_{l, r}(x, \lambda)\right| \text { and }\left|R_{l}(x, \lambda)\right|<\left(\frac{1}{|l+\lambda|}+\frac{1}{|l-\lambda|}\right) A \text {. }
$$

Thus, for $\epsilon(>0)$ however small,

$$
\left|R_{l_{e}, r}(x, \lambda)\right|<\epsilon / 3, \quad\left|R_{l_{e}}(x, \lambda)\right|<\epsilon / 3,
$$

provided $l_{c}$ is taken sufficiently great. We have, for $x$ and $\lambda$ fixed $(\beta \neq 0)$,

$$
\begin{array}{r}
\left|\int_{-\infty}^{\infty} \frac{1}{\mu-\lambda} d_{\mu} p(x, \mu)-\int_{-\infty}^{\infty} \frac{1}{\mu-\lambda} \dot{d}_{\mu} p_{r}(x, \mu)\right|=\mid R_{l_{\epsilon}}(x, \lambda)-R_{l_{e}, r}(x, \lambda) \\
+\left[\int_{-l}^{l} \frac{1}{\mu-\lambda} d_{\mu} p(x, \mu)-\int_{-l}^{l} \frac{1}{\mu-\lambda} d_{\mu} p_{r}(x, \mu)\right] \mid<\epsilon,
\end{array}
$$

provided $l=l_{\epsilon}$ is such that $(5.8 \mathrm{a})$ holds and provided $r=r_{\epsilon}(x, \lambda)$ is taken sufficiently great (cf. (5.8)). This establishes (5.6).

We come now to the consideration of (3.20). In consequence of (3.20), applied to $\Omega_{1}^{\delta_{0 . r}}$, it is concluded that a solution of (5.2) may be given in the form

$$
\phi_{1}^{\delta_{0}, r}(x)=f(x)+\lambda \frac{\partial}{\partial x} \int_{-\infty}^{\infty} \frac{1}{\mu-\lambda} d_{\mu} \int_{a}^{b} f(y) \frac{\partial}{\partial y} \Omega_{\mathbb{1}}^{\delta_{0, r}}(x, y \mid \mu) d y
$$

with

$$
\int_{a}^{b}\left|\phi_{1}^{\delta_{0}, r}(x)\right|^{2} d x \leqq M .
$$

In virtue of (5.10a) and with the aid of a reasoning of the type previously employed in connection with (5.3)-(5.5a) it is concluded that 


$$
\lim _{r} \phi_{1}^{\delta_{0}, r}(x)=\phi_{1}(x), \quad \int_{a}^{b}\left|\phi_{1}(x)\right|^{2} d x \leqq M
$$

(suitable $\delta_{0, r}(r=1,2, \cdots)$; convergence in the weak sense), where $\phi_{1}(x)$ is a solution of (5.1). Now (5.11) implies that (cf. (5.10))

$$
\begin{aligned}
\int_{a}^{x}{\phi_{1}}^{\delta_{0}, r}(x) d x & =\int_{a}^{x} f(x) d x+\lambda \int_{-\infty}^{\infty} \frac{1}{\mu-\lambda} d_{\mu} \int_{a}^{b} f(y) \frac{\partial}{\partial y} \Omega_{1}^{\delta_{0}, r}(x, y \mid \mu) d y \\
& \rightarrow H(x) \quad[\text { as } r \rightarrow \infty ; H(x) \text { absolutely continuous], }
\end{aligned}
$$

where

$$
\frac{d}{d x} H(x)=\phi_{1}(x) \quad\left[\phi_{1}(x) \text { from (5.11); almost everywhere }\right] .
$$

Clearly, because of (5.6),

$$
H(x)=\int_{a}^{x} f(x) d x+\lambda \int_{-\infty}^{\infty} \frac{1}{\mu-\lambda} d_{\mu} \int_{a}^{b} f(y) \frac{\partial}{\partial y} \Omega_{1}(x, y \mid \mu) d y .
$$

From (5.11a) and (5.11b) it is deduced that $\phi_{1}(x)$ is represented by the formula (3.20) (with $\Omega=\Omega_{1}$ ). On taking account of (5.11) it is finally concluded that the italicized statement made in connection with (3.20) holds for the class $H_{m}$.

In accordance with (3.21) we write

$$
\begin{array}{r}
\psi_{1}\left(x, l \mid \delta_{0}, \cdots, \delta_{m-1}\right) \\
=\left(\int_{l}^{\infty}+\int_{-\infty}^{-l}\right) \frac{1}{\mu} d_{\mu} \int_{a}^{b} \theta_{1}^{\delta_{0}}, \cdots, \delta_{m-1}(x, y \mid \mu) h(y) d y \\
{\left[h(y) \subset L_{2} ; \theta_{1}^{\delta_{0}}, \cdots, \delta_{m-1}=\text { spectrum of } K_{1}^{\left.\delta_{0}, \cdots, \delta_{m-1}(x, y)\right] .}\right.}
\end{array}
$$

By (3.21a), applied to $\psi_{1}$ of (5.12), one may assert only the following:

$$
\begin{aligned}
\psi_{1}\left(x, l \mid \delta_{0}, \cdots, \delta_{m-1, r}\right) & \rightarrow \psi_{1}\left(x, l \mid \delta_{0}, \cdots, \delta_{m-2}\right), \\
\psi_{1}\left(x, l \mid \delta_{0}, \cdots, \delta_{m-2, r}\right) & \rightarrow \psi_{1}\left(x, l \mid \delta_{0}, \cdots, \delta_{m-3}\right), \cdots, \\
\psi_{1}\left(x, l \mid \delta_{0}, \delta_{1, r}\right) & \rightarrow \psi_{1}\left(x, l \mid \delta_{0}\right),
\end{aligned}
$$

the $\delta_{\nu, r}(\nu=m-1, \cdots, 1 ; r=1,2, \cdots)$ being suitably chosen and convergence being in the weak sense in $x$ (as $r \rightarrow \infty$ ); moreover, in consequence of (3.21b) and (3.21c)

$$
\begin{gathered}
\int_{a}^{b} \psi_{1}^{2}\left(x, l \mid \delta_{0}\right) d x \leqq \frac{1}{l^{2}} \int_{a}^{b} h^{2}(x) d x, \\
\lim _{l} L_{x}^{\prime}\left(\xi \mid \psi_{1}\left(x, l \mid \delta_{0, r}\right)\right)=0 .
\end{gathered}
$$

In virtue of $(5.12 \mathrm{~b})$ with the aid of Theorem 1.3 it is deduced that 


$$
\lim _{r} \psi_{1}\left(x, l \mid \delta_{0, r}\right)=\psi_{1}(x, l)
$$

[convergence in the weak sense; suitable $\delta_{0, r}(>0) \rightarrow 0$ ],

$$
\int_{a}^{b} \psi_{1}^{2}(x, l) d x \leqq \frac{1}{l^{2}} \int_{a}^{b} h^{2}(x) d x .
$$

From (5.13a) it is inferred that

$$
\left|\int_{a}^{x} \psi_{1}(x, l) d x\right| \leqq(b-a)^{1 / 2}\left[\int_{a}^{x} \psi_{1}^{2}(x, l) d x\right]^{1 / 2} \leqq \frac{1}{l} A,
$$

so that

$$
\lim _{l} \int_{a}^{x} \psi_{1}(x, l) d x=0
$$

Thus, $\psi_{1}(x, l)$ converges weakly (in $x$ ) to zero, as $l \rightarrow \infty$. Hence, in view of property $\left(2.23^{\prime}\right)$

$$
\lim _{l} L^{\prime}\left(\xi \mid \psi_{1}(x, l)\right)=L^{\prime}(\xi \mid 0)=0 .
$$

The relations (5.13), (5.13a), (5.13b) imply that the statements made in connection with (3.21)-(3.21c) hold true for the class $H_{m}$.

By (3.22), applied to the kernel $K_{1}^{\delta_{0}, r}(x, y)$,

$$
\begin{aligned}
& \int_{a}^{b} L_{x}^{\prime}\left(\xi \mid K_{1}^{\delta_{0}, r}(x, y)\right) h(y) d y \\
& =\int_{-l}^{l} d_{\mu} \int_{a}^{b} L_{x}^{\prime}\left(\xi \mid K_{1}^{\delta_{0}, r}(x, s)\right)\left[\frac{\partial}{\partial s} \int_{a}^{b} h(t) \frac{\partial}{\partial t} \Omega_{1}^{\delta_{0}, r}(s, t \mid \mu) d t\right] d s \\
& +L_{x}^{\prime}\left(\xi \mid \psi_{1}\left(x, l \mid \delta_{0, r}\right)\right) \\
& \left(\psi_{1}\left(x, l \mid \delta_{0}\right)\right. \text { from (5.12a)). }
\end{aligned}
$$

In the limit, as $r \rightarrow \infty$ (the $\delta_{0, r}$ being suitably chosen) we get

$$
\begin{aligned}
\int_{a}^{b} L_{x}^{\prime}(\xi \mid & \left.K_{1}(x, y)\right) h(y) d y \\
= & \int_{-l}^{l} d_{\mu} \int_{a}^{b} L_{x}^{\prime}\left(\xi \mid K_{1}(x, s)\right)\left[\frac{\partial}{\partial s} \int_{a}^{b} h(t) \frac{\partial}{\partial t} \Omega_{1}(s, t \mid \mu) d t\right] d s \\
& +L_{x}^{\prime}\left(\xi \mid \psi_{1}(x, l)\right) \\
& \left(\psi_{1}(x, l) \text { from }(5.13)\right) .
\end{aligned}
$$

In fact, the first member of (5.14a) is obtained as a consequence of (3), (1), (2) and of Theorem 1.2, where we put $g_{r}(y)=h(y)$ and

$$
f_{r}(y)=L_{x}^{\prime}\left(\xi \mid K_{1}^{\delta_{0}, r}(x, y)\right), \quad \gamma(y)=\gamma(\xi \mid y) .
$$

The integral displayed in the $2 \mathrm{~d}$ member of $(5.14 \mathrm{a})$ is obtained from the corre- 
sponding integral in (5.14) with the aid of the following considerations. Since

$$
\begin{aligned}
p_{r}(s, \mu) & =\frac{\partial}{\partial s} \int_{a}^{b} h(t) \frac{\partial}{\partial t} \Omega_{1}^{\delta_{0}, r}(s, t \mid \mu) d t \\
& \rightarrow \frac{\partial}{\partial s} \int_{a}^{b} h(t) \frac{\partial}{\partial t} \Omega_{1}(s, t \mid \mu) d t=p(s, \mu),
\end{aligned}
$$

convergence being in the weak sense in $s$, and since

$$
\begin{aligned}
L_{x}\left(\xi \mid K_{1}^{\delta_{0}, r}(x, s)\right) & \rightarrow L_{x}^{\prime}\left(\xi \mid K_{1}(x, s)\right) & (\text { as } r \rightarrow \infty) \\
\left|L_{x}^{\prime}\left(\xi \mid K_{1}^{\delta_{0}, r}(x, s)\right)\right| & \leqq \gamma(\xi \mid s) \subset L_{2} & \text { (in } s),
\end{aligned}
$$

by Theorem 1.5 it is inferred that

$$
\begin{aligned}
q_{r}(\mu) & =\int_{a}^{b} L_{x}^{\prime}\left(\xi \mid K_{1}^{\delta_{0, r}}(x, s)\right) p_{r}(s, \mu) d s \\
& \rightarrow \int_{a}^{b} L_{x}^{\prime}\left(\xi \mid K_{1}(x, s)\right) p(s, \mu) d s=q(\mu) \quad(\text { as } r \rightarrow \infty) .
\end{aligned}
$$

Moreover, by (3.11a) with

$$
g(s)=L_{x}^{\prime}\left(\xi \mid K_{1}^{\delta_{0}, r}(x, s)\right), \quad \Omega=\Omega_{1}^{\delta_{0, r},}
$$

and in view of (3), (2), it is concluded that

$$
\begin{aligned}
\underset{\mu}{\operatorname{Var} .} q_{r}(\mu) & \leqq\left[\int_{a}^{b} h^{2}(x) d x\right]^{1 / 2}\left[\int_{a}^{b}\left|L_{x}^{\prime}(\xi) K_{1}^{\delta_{0}, r}(x, s)\right|^{2} d s\right]^{1 / 2} \\
& \leqq\left[\int_{a}^{b} h^{2}(x) d x\right]^{1 / 2}\left[\int_{a}^{b} \gamma^{2}(\xi \mid s) d s\right]^{1 / 2}=A(\xi)
\end{aligned}
$$

where $A(\xi)$ is independent of $r$ and $\mu$. In consequence of (5.15) and (5.15a), application of Theorem 1.1 is possible, yielding the result

$$
\int_{-l}^{l} d q_{r}(\mu) \rightarrow \int_{-l}^{l} d q(\mu)
$$

which accounts for the integral in the second member of (5.14a). With $\psi_{1}\left(x, l \mid \delta_{0, r}\right)$ converging weakly (in $x$, as $\left.r \rightarrow \infty\right)$ to $\psi_{1}(x, l)$, we have

$$
\lim _{r} L_{x}^{\prime}\left(\xi \mid \psi_{1}\left(x, l \mid \delta_{0, r}\right)\right)=L_{x}^{\prime}\left(\xi \mid \psi_{1}(x, l)\right)
$$

in view of the condition (4). Accordingly, one may consider (5.14a) established.

On letting $l$ in (5.14a) approach infinity, in consequence of (5.13b) it is inferred that (cf. (5.15)) 


$$
\int_{a}^{b} L_{x}^{\prime}\left(\xi \mid K_{1}(x, y)\right) h(y) d y=\int_{-\infty}^{\infty} d_{\mu} q(\mu) .
$$

Accordingly, we observe that (3.22) holds for the class $H_{m}$.

In accordance with (3.23) write

$$
\begin{aligned}
\tau_{r}^{\prime}(x, l) & =\frac{\partial}{\partial x} \int_{-l}^{l} d_{\lambda} \int_{a}^{b} f(y) \frac{\partial}{\partial y} \Omega_{1}^{\delta_{0}, r}(x, y \mid \lambda) d y, & \\
w_{r}^{\prime}(x, l) & =f(x)-\tau_{r}^{\prime}(x, l) & \left(f(x) \subset L_{2}\right) .
\end{aligned}
$$

By $(3.23 \mathrm{a})-(3.23 \mathrm{c})$

$$
\begin{gathered}
\int_{a}^{b} \tau_{r}^{\prime 2}(x, l) d x \leqq q=\int_{a}^{b} f^{2}(x) d x \\
w_{r}^{\prime}\left(x, l_{\nu}\right) \rightarrow w_{r}^{\prime}(x) \quad\left(\text { as } l_{\nu} \rightarrow \infty\right), \quad \int_{a}^{b} w_{r}^{\prime 2}(x) d x \leqq 4 q,
\end{gathered}
$$

convergence being in the weak sense; moreover, in view of (3.23d)

$$
\int_{a}^{b} L_{x}^{\prime}\left(\xi \mid K_{1}^{\delta_{0}, r}(x, y)\right) w_{r}^{\prime}(y) d y=0 .
$$

Let $\tau^{\prime}(x, l)$ be $\tau_{r}^{\prime}(x, l)$, with $\delta_{0, r}$ in the integrand of (5.16) deleted, and let $w^{\prime}(x, l)=f(x)-\tau^{\prime}(x, l)$. Then because of (3.13), applied with $\alpha(\lambda)=1$ to $\Omega_{1}$, one has

$$
\int_{a}^{b} \tau^{\prime 2}(x, l) d x \leqq q,^{*}
$$

in consequence of which

$$
\int_{a}^{b} w^{\prime 2}(x, l) d x \leqq 4 q .
$$

By Theorem 1.3 the latter inequality implies that there exists a subsequence $\left(0<l_{1}^{\prime}<l_{2}^{\prime} ; \lim _{\nu} l_{\nu}^{\prime}=\infty\right)$ such that

$$
w^{\prime}\left(x, l_{\nu}^{\prime}\right) \rightarrow w^{\prime}(x) \quad(\text { as } \nu \rightarrow \infty), \quad \int_{a}^{b} w^{\prime 2}(x) d x \leqq 4 q,
$$

convergence being in the weak sense (in $x$ ). The function $w^{\prime}(x)$ can also be obtained by a limiting process with the aid of (5.16) and of the last inequality (5.16a); we obtain (cf. Theorem 1.3)

* (5.16c) can also be obtained by a limiting process applied on the basis of (5.16a), with the aid of Theorem 1.3. 
(5.16e) $\quad w_{r}^{\prime}(x) \rightarrow w^{\prime}(x) \quad$ [as $r \rightarrow \infty$; weak convergence in $x$; suitable $\delta_{0, r}$ ]. In view of (5.16e) and since (by $\left(2.22^{\prime}\right)$ and $\left.\left(2.21^{\prime}\right)\right)$

$$
\begin{aligned}
L_{x}^{\prime}\left(\xi \mid K_{1}^{\delta_{0}, r}(x, y)\right) & \rightarrow L_{x}^{\prime}\left(\xi \mid K_{1}(x, y)\right), \\
\left|L_{x}^{\prime}\left(\xi \mid K_{1}^{\delta_{0}, r}(x, y)\right)\right| & \leqq \gamma(\xi \mid x) \subset L_{2},
\end{aligned}
$$

application of Theorem 1.5 to the first member of (5.16b) is possible; thus,

$$
\int_{a}^{b} L_{x}^{\prime}\left(\xi \mid K_{1}(x, y)\right) w^{\prime}(y) d y=0 .
$$

Hence it is observed that the statements previously made in connection with (3.23)-(3.23d) will hold for the class $H_{m}$.

If the only solution (included in $L_{2}$ ) of the equation (3.24) [with $L=L^{\prime}$ and $K=K_{1}$ ] is $\phi(y)=0$ (almost everywhere), then in consequence of (5.17), $w^{\prime}(y)=0$. Now, according to the statement subsequent to $(5.16 \mathrm{~b})$

$$
w^{\prime}\left(x, l_{\nu}^{\prime}\right)=f(x)-\tau^{\prime}\left(x, l_{\nu}^{\prime}\right)
$$

thus, by (5.16d),

$$
\tau^{\prime}\left(x, l_{\nu}^{\prime}\right) \rightarrow f(x) \quad(\text { as } \nu \rightarrow \infty \text {; in the weak sense). }
$$

That is, in view of (5.16) (with $\delta_{0, r}$ deleted)

$$
\int_{a}^{x} \tau^{\prime}\left(x, l_{\nu}^{\prime}\right) d x=\int_{-l_{\nu}^{\prime}}^{l_{\nu}^{\prime}} d_{\lambda} \int_{a}^{b} f(y) \frac{\partial}{\partial y} \Omega_{1}(x, y \mid \lambda) d y \rightarrow \int_{a}^{x} f(x) d x(\text { as } \nu \rightarrow \infty) .
$$

Hence

$$
\int_{-\infty}^{\infty} d_{\lambda} \int_{a}^{b} f(y) \frac{\partial}{\partial y} \Omega_{1}(x, y \mid \lambda) d y=\int_{a}^{x} f(x) d x .^{*}
$$

This formula implies the representation (3.24a), as slated, for the class $H_{m}$.

With $\Delta$ designating the operation indicated preceding (3.25) it is observed that, by (3.9a) (for $\Omega=\Omega_{1}$ ), we have in the sense of weak convergence (in $x$ )

$$
\frac{\partial}{\partial x} \Delta \int_{y=a}^{b} h(y) \frac{\partial}{\partial y} \Omega_{1}^{\delta_{0}, r}(x, y \mid \lambda) d y \rightarrow \frac{\partial}{\partial x} \Delta \int_{y=a}^{b} h(y) \frac{\partial}{\partial y} \Omega_{1}(x, y \mid \lambda) d y ;
$$

(3.9) will hold for $\Omega_{1}^{\delta_{0}, r}$. From (4) it is deduced that

$$
\begin{aligned}
\lim _{r} L_{x}\left(\xi \mid \frac{\partial}{\partial x} \Delta \int_{a}^{b} h(y) \frac{\partial}{\partial y}\right. & \left.\Omega_{1}^{\delta_{0}, r}(x, y \mid \lambda) d y\right) \\
& =L_{x}\left(\xi \mid \frac{\partial}{\partial x} \Delta \int_{a}^{b} h(y) \frac{\partial}{\partial y} \Omega_{1}(x, y \mid \lambda) d y\right) .
\end{aligned}
$$

* The integral of the first member is convergent. 
It is noted that

$$
q_{r}(s)=\frac{\partial}{\partial s} \int_{\lambda^{\prime}}^{\lambda^{\prime \prime}} \mu d_{\mu} \int_{a}^{b} h(y) \frac{\partial}{\partial y} \Omega_{1}^{\delta_{0}, r(s, y \mid \mu) d y \subset L_{2}}
$$

and that (with $\delta_{0, r}$ suitably chosen)

(5.19a) $\quad \lim _{r} q_{r}(s)=\frac{\partial}{\partial s} \int_{\lambda^{\prime}}^{\lambda^{\prime \prime}} \mu d_{\mu} \int_{a}^{b} h(y) \frac{\partial}{\partial y} \Omega_{1}(s, y \mid \mu) d y=q(s)$

(weak convergence). In fact, (5.19) follows from (3.13) (with $\alpha(\mu)=\mu$ and $\Omega=\Omega_{1} \delta_{0, r}$ ), while (5.19a) is a consequence of (3.13), (3.12) [with $\alpha(\mu)=\mu$ and $g=1$ on $(a, x), g=0$ on $(x, b)]$ and of Theorem 1.3; we have (cf. (3.13))

$$
\int_{a}^{b} q^{2}(s) d s \leqq M^{2} \int_{a}^{b} h^{2}(s) d s .
$$

In virtue of (5.19), (5.19a), and (5.16f) from Theorem 1.5 it is inferred that (5.20) $\lim _{r} \int_{a}^{b} L_{x}^{\prime}\left(\xi \mid K_{1}^{\delta_{0}, r}(x, s)\right) q_{r}(s) d s=\int_{a}^{b} L_{x}^{\prime}\left(\xi \mid K_{1}(x, s)\right) q(s) d s$.

With (5.18) and (5.20) in view, write (3.25) for $\Omega=\Omega_{1}^{\delta_{0, r}, L}=L^{\prime}$, and $K=K_{1}^{\delta_{0, r}}$ and pass to the limit, thus obtaining the formula

$$
\begin{aligned}
L_{x}^{\prime}\left(\xi \mid \frac{\partial}{\partial x} \Delta \int_{a}^{b} h(y) \frac{\partial}{\partial y} \Omega_{1}(x, y \mid \lambda) d y\right) \\
-\int_{a}^{b} L_{x}^{\prime}\left(\xi \mid K_{1}(x, s)\right) q(s) d s=0 ;
\end{aligned}
$$

accordingly, it is observed that (3.25) holds for the class $H_{m}$, the same being true for (3.25a) (which is obtained from (3.25) by specializing $h$ ).

The proof of the important formula (3.26) (for the class $H_{m}$ ) can be effected as follows. In view of (3.22) (with $L=L^{\prime}, K=K_{1}, \Omega=\Omega_{1}$ ), (3.26) will hold, for $L=L^{\prime}, K=K_{1}, \Omega=\Omega_{1}$, provided

$$
\begin{aligned}
\int_{-\infty}^{\infty} d_{\mu} \int_{a}^{b} L_{x}^{\prime}\left(\xi \mid K_{1}(x, s)\right)\left[\frac{\partial}{\partial s} \int_{a}^{b} h(y) \frac{\partial}{\partial y} \Omega_{1}(s, y \mid \mu) d y\right] d s \\
=\int_{-\infty}^{\infty} \frac{1}{\mu} d_{\mu} L_{x}^{\prime}\left(\xi \mid \frac{\partial}{\partial x} \int_{a}^{b} h(y) \frac{\partial}{\partial y} \Omega_{1}(x, y \mid \mu) d y\right)=N(\xi) .
\end{aligned}
$$

On writing

$$
N_{l}(\xi)=\int_{-l}^{l} \frac{1}{\mu} d_{\mu} L_{x}^{\prime}\left(\xi \mid \frac{\partial}{\partial x} \int_{a}^{b} h(y) \frac{\partial}{\partial x} \Omega_{1}(x, y \mid \mu) d y\right),
$$


and

$$
-l=\lambda_{0}<\lambda_{1}<\cdots<\lambda_{m_{1}}=l \quad\left(\Delta_{\nu} \text { corresponding to }\left(\lambda_{\nu-1}, \lambda_{\nu}\right)\right),
$$

we have

$$
N_{l}(\xi)=\lim _{m_{1}} N_{l, m_{1}}(\xi)
$$

where

(5.21c) $\quad N_{l, m_{1}}(\xi)=\sum_{\nu=1}^{m_{1}} \frac{1}{\mu_{\nu}} L_{x}^{\prime}\left(\xi \mid \frac{\partial}{\partial x} \Delta_{\nu} \int_{a}^{b} h(y) \frac{\partial}{\partial y} \Omega_{1}(x, y \mid \mu) d y\right)$

$\left(\mu_{\nu}\right.$ in $\left.\left(\lambda_{\nu-1}, \lambda_{\nu}\right)\right)$. By (3.25) (for $\left.L^{\prime}, K_{1}, \Omega_{1}\right)$ and (5.21c)

$$
\begin{aligned}
N_{l, m_{1}}(\xi)=\sum_{\nu=1}^{m_{1}} \frac{1}{\mu_{\nu}} \int_{a}^{b} L_{x}^{\prime}\left(\xi \mid K_{1}(x, s)\right) \\
\cdot\left[\frac{\partial}{\partial s} \int_{\lambda_{\nu-1}}^{\lambda_{\nu}} \mu d_{\mu} \int_{a}^{b} h(y) \frac{\partial}{\partial y} \Omega_{1}(s, y \mid \mu) d y\right] d s .
\end{aligned}
$$

Applying to the integral displayed in (5.21d) the first identity (3.14), with

$$
g(s)=L_{x}^{\prime}\left(\xi \mid K_{1}(x, s)\right), \quad \alpha(\mu)=\mu, \quad \Omega=\Omega_{1},
$$

it is concluded that (5.21d) may be written in the form

$$
\begin{aligned}
N_{l, m_{1}}(\xi) & =\sum_{\nu=1}^{m_{1}} \frac{1}{\mu_{\nu}} \int_{\lambda_{\nu-1}}^{\lambda_{\nu}} \mu \\
\cdot & d_{\mu}\left\{\int_{a}^{b} L_{x}^{\prime}\left(\xi \mid K_{1}(x, s)\right)\left[\frac{\partial}{\partial s} \int_{a}^{b} h(y) \frac{\partial}{\partial y} \Omega_{1}(s, y \mid \mu) d y\right] d s\right\} .
\end{aligned}
$$

Thus

$$
N_{l, m_{1}}(\xi)=\sum_{\nu=1}^{m_{1}} \frac{1}{\mu_{\nu}} \Delta_{\nu} \gamma(\mu), \quad \gamma(\mu)=\int_{-l}^{\mu} \mu d_{\mu}\{\cdots\},
$$

and, in view of $(5.21 \mathrm{~b})$,

$$
N_{l}(\xi)=\int_{-l}^{l} \frac{1}{\mu} d_{\mu} \gamma(\mu) .
$$

By definition of $\gamma(\mu)$ (cf. (5.21f)), with $\{\cdots\}$ from (5.21e), we have

$$
\begin{aligned}
N_{l}(\xi) & =\int_{-l}^{l} \frac{1}{\mu}\left[\mu d_{\mu}\{\cdots\}\right] \\
& =\int_{-l}^{l} d_{\mu}\left\{\int_{a}^{b} L_{x}^{\prime}\left(\xi \mid K_{1}(x, s)\right)\left[\frac{\partial}{\partial s} \int_{a}^{b} h(y) \frac{\partial}{\partial y} \Omega_{1}(s, y \mid \mu) d y\right] d s\right\} .
\end{aligned}
$$


By (5.22a), (5.21a)

$$
\lim _{l} N_{l}(\xi)=N(\xi)=\text { first member of }(5.21) \text {. }
$$

Whence it is observed that (5.21) and consequently (3.26) (for $L^{\prime}, K_{1}, \Omega_{1}$ ) have been established.

We shall now proceed to prove the statement in connection with (3.27) for the class $H_{m}$. The identity to be proved is

$$
\begin{aligned}
& L_{x}^{\prime}\left(\xi \mid \frac{\partial}{\partial x} \int_{\lambda^{\prime}}^{\lambda^{\prime \prime}} \mu d_{\mu} \Omega_{1}(x, y \mid \mu)\right) \\
& -\lambda \int_{a}^{b} L_{x}^{\prime}\left(\xi \mid K_{1}(x, s)\right)\left[\frac{\partial}{\partial s} \int_{\lambda^{\prime}}^{\lambda^{\prime \prime}} \mu d_{\mu} \Omega_{1}(s, y \mid \mu)\right] d s \\
& =L_{x}^{\prime}\left(\xi \mid \frac{\partial}{\partial x} \int_{\lambda^{\prime}}^{\lambda^{\prime \prime}}(\mu-\lambda) d_{\mu} \Omega_{1}(x, y \mid \mu)\right) .
\end{aligned}
$$

In view of (3.25a) (for $\left.L^{\prime}, K_{1}, \Omega_{1}\right),(5.23 \mathrm{a})$ will hold if

$L_{x}^{\prime}\left(\xi \mid \frac{\partial}{\partial x} \int_{\lambda^{\prime}}^{\lambda^{\prime \prime}} \mu d_{\mu} \Omega_{1}(x, y \mid \mu)\right)-\lambda L_{x}^{\prime}\left(\xi \mid \frac{\partial}{\partial x} \Delta \Omega_{1}(x, y \mid \lambda)\right)$

that is, if

$$
=L_{x}^{\prime}\left(\xi \mid \frac{\partial}{\partial x} \int_{\lambda^{\prime}}^{\lambda^{\prime \prime}}(\mu-\lambda) d_{\mu} \Omega_{1}(x, y \mid \mu)\right) \text {; }
$$

$$
L_{x}^{\prime}\left(\xi \mid \frac{\partial}{\partial x} \Delta \Omega_{1}(x, y \mid \lambda)\right)=L_{x}^{\prime}\left(\xi \mid \frac{\partial}{\partial x} \int_{\lambda^{\prime}}^{\lambda^{\prime \prime}} d_{\mu} \Omega_{1}(x, y \mid \mu)\right) .
$$

Now, (5.23a) holds since

$$
\Delta \Omega_{1}(x, y \mid \lambda)=\int_{\lambda^{\prime}}^{\lambda^{\prime \prime}} d_{\mu} \Omega_{1}(x, y \mid \mu) .
$$

Thus, the result previously stated with respect to (3.27) holds for the class $H_{m}$.

The developments of this section may be summed in the theorem:

TheORem 5.1. Suppose classes $H_{n}$ are specified by Definition 2.2. For every finite $m(>0)$ the following will hold. If $K(x, y) \subset H_{m}$ and if "associated" with $K(x, y)$ there is an operator $L$ (cf. Definition 2.3), then the statements made in $\$ 3$ will hold true with respect to this kernel and this operator.

6. Kernels of class $H_{1}$. For kernels of class $H_{1}$ with which operators $L$ (Definition 2.3) can be "associated," as remarked by Carleman many results 
of (C, chap. 2) can be extended. In view of the purpose to examine the possibility of such extension to our classes $H_{n}$ it will be essential to investigate in some detail the situation with respect to $H_{1}$.

Thus, suppose $K(x, y) \subset H_{1}$, an operator $L$ being "associated" with $K(x, y)$.

We write down the equations

$$
\phi^{\delta_{0}}(x)-\lambda \int_{a}^{b} K^{\delta_{0}}(x, y) \phi^{\delta_{0}}(y) d y=f(x),
$$

$$
\begin{gathered}
L_{x}\left(\xi \mid \phi^{\delta_{0}}(x)\right)-\lambda \int_{a}^{b} L_{x}\left(\xi \mid K^{\delta_{0}}(x, y)\right) \phi^{\delta_{0}}(y) d y=L_{x}(\xi \mid f(x)), \\
L_{x}(\xi \mid \phi(x))-\lambda \int_{a}^{b} L_{x}(\xi \mid K(x, y)) \phi(y) d y=L_{x}(\xi \mid f(x)), \\
L_{x}(\xi \mid \phi(x))-\lambda \int_{a}^{b} L_{x}(\xi \mid K(x, y)) \phi(y) d y=0 .
\end{gathered}
$$

As indicated in (C), if $\phi^{\delta_{0}, r}(x)$ is a solution of (6.1) (for $\delta=\delta_{0, r}$ suitably chosen; $r=1,2, \cdots ; \lim \delta_{0, r}=0$ ), then $\phi^{\delta_{0}, r}(x) \rightarrow \phi(x)$ (weakly in $x$ ) and (3.19a) will hold; moreover, $\phi(x)$ will be a solution of (6.2). On writing, conforming with (C),

$$
\phi^{\delta_{0}}(x)=\frac{i \lambda^{\prime}}{2 \beta}\left[f(x)+\psi^{\delta_{0}}(x)\right], \quad \phi(x)=\frac{i \lambda^{\prime}}{2 \beta}[f(x)+\psi(x)],
$$

where $\lambda^{\prime}$ is the conjugate of $\lambda$, we conclude that

$$
\int_{a}^{b}\left|\psi^{\delta_{0}, r(x)}\right|^{2} d x=\int_{a}^{b}|f(x)|^{2} d x
$$

and, in the limit,

$$
\int_{a}^{b}|\psi(x)|^{2} d x \leqq \int_{a}^{b}|f(x)|^{2} d x .
$$

It is observed that (6.3a) can be established with the aid of the relation, found in (C),

$$
\begin{aligned}
\frac{-\beta}{|\lambda|^{2}} \int_{a}^{b}\left|\phi^{\delta_{0}}(x)\right|^{2} d x= & \frac{1}{2 i \lambda} \int_{a}^{b} f(x) \phi_{1}^{\delta_{0}}(x) d x \\
& -\frac{1}{2 i \lambda^{\prime}} \int_{a}^{b} \bar{f}(x) \phi^{\delta_{0}}(x) d x .
\end{aligned}
$$

Here and in the sequel, $\phi_{1}$ denotes the conjugate of $\phi$.

We shall prove the following fact. In order that (6.3b) should hold (when 
$\Pi \lambda=\beta \neq 0)$ with the equality sign it is necessary and sufficient that

$$
\lim \int_{a}^{b}\left|\phi^{\delta_{0}, r(x)}\right|^{2} d x=\int_{a}^{b}|\phi(x)|^{2} d x .
$$

In fact, it is noted that

$$
\lim \int_{a}^{b} f(x) \phi_{1}^{\delta_{0}, r}(x) d x=\int_{a}^{b} f(x) \phi(x) d x,
$$

inasmuch as $f(x) \subset L_{2}$ and other conditions of Theorem 1:4 hold. Thus, by (6.3c)

$$
\begin{aligned}
\frac{-\beta}{|\lambda|^{2}} \lim \int_{a}^{b}\left|\phi^{\delta_{0}, r}(x)\right|^{2} d x= & \frac{1}{2 i \lambda} \int_{a}^{b} f(x) \phi_{1}(x) d x \\
& -\frac{1}{2 i \lambda^{\prime}} \int_{a}^{b} \bar{f}(x) \phi(x) d x=\gamma,
\end{aligned}
$$

and, if (6.4) holds, there will be on hand an equality like (6.3c) with $\phi^{\delta_{0}}(x)$ replaced by $\phi(x)$; from this relation with the aid of the second one of (6.3) we obtain (6.3b) with the equality sign. Thus (6.4) is a sufficient condition. If, on the other hand, (6.4) does not hold, it is observed that, inasmuch as the limit in (6.4a) exists,

$$
\lim \int_{a}^{b}\left|\phi^{\delta_{0}, r(x)}\right|^{2} d x=\frac{\gamma|\lambda|^{2}}{-\beta}=\left|\frac{\gamma}{\beta}\right||\lambda|^{2}>\int_{a}^{b}|\phi(x)|^{2} d x ;
$$

this inequality follows by a theorem of $F$. Riesz according to which

$$
\lim \sup \int_{a}^{b}\left|f_{\nu}(x)\right|^{2} d x \geqq \int_{a}^{b}|f(x)|^{2} d x,
$$

whenever $f_{\nu}(x) \subset L_{2}$ and $f_{\nu}(x) \rightarrow f(x)$ (in the weak sense). Now, in consequence of $(6.4 \mathrm{~b})$

$$
\frac{|\beta|}{|\lambda|^{2}} \int_{a}^{b}|\phi(x)|^{2} d x<|\gamma| \quad \quad(\gamma \text { from (6.4a)); }
$$

substitution of $\phi(x)$ from (6.3) and (6.4c) will result in (6.3b), with the inequality sign. Hence the statement in connection with (6.4) is seen to be true.* $^{*}$

Of special interest appear to be operators $L$, which in addition to the condi-

* The corresponding result in (C) depends on the possibility of interchange of order of integration in a certain double integral. 
tions of Definition 2.3 satisfy the following. $L$ is defined for $\xi$ in a set $\Gamma$ dense in itself; moreover, for $\xi_{1}, \xi_{2}$, in $\Gamma$,

$$
\int_{a}^{b}\left|L_{x}\left(\xi_{1} \mid K^{\delta_{0}}(x, y)\right)-L_{x}\left(\xi_{2} \mid K^{\delta_{0}}(x, y)\right)\right|^{2} d y \leqq G\left(\xi_{1}, \xi_{2}\right),
$$

where $G\left(\xi_{1}, \xi_{2}\right)$ is independent of $\delta_{0}$ and

$$
G\left(\xi_{1}, \xi_{2}\right) \rightarrow 0 \quad\left(\text { as } \xi_{1}-\xi_{2} \rightarrow 0 ; \xi_{1}, \xi_{2} \text { in } \Gamma\right) .
$$

In consequence of (2.22) the limit of the integrand (for $\delta_{0} \rightarrow 0$ ) in (6.5) exists; by (2.21) the integrand is less than

$$
\left[\gamma\left(\xi_{1} \mid y\right)+\gamma\left(\xi_{2} \mid y\right)\right]^{2} \subset L_{1}
$$

for $\xi_{1}, \xi_{2}$ on $\Gamma$. Hence by passage to the limit, from (6.5) it is inferred that

$$
\int_{a}^{b}\left|L_{x}\left(\xi_{1} \mid K(x, y)\right)-L_{x}\left(\xi_{2} \mid K(x, y)\right)\right|^{2} d y \leqq G\left(\xi_{1}, \xi_{2}\right) .
$$

Let $\phi(x)$ be a solution of (6.2) obtained by a limiting process as indicated subsequent to (6.2a). Then, by (6.2) and by the inequality of Schwartz,

$$
\begin{aligned}
\mid L_{x}\left(\xi_{1} \mid \phi(x)\right. & -f(x))-\left.L_{x}\left(\xi_{2} \mid \phi(x)-f(x)\right)\right|^{2} . \\
& =|\lambda|^{2}\left|\int_{a}^{b}\left[L_{x}\left(\xi_{1} \mid K(x, y)\right)-L_{x}\left(\xi_{2} \mid K(x, y)\right)\right] \phi(y) d y\right|^{2} \\
& \leqq|\lambda|^{2} \int_{a}^{b}|\phi(y)|^{2} d y \int_{a}^{b}\left|L_{x}\left(\xi_{1} \mid K(x, y)\right)-L_{x}\left(\xi_{2} \mid K(x, y)\right)\right|^{2} d y .
\end{aligned}
$$

If (6.5) and (6.5a) hold, then in consequence of (6.5b) and of (3.19a)

$$
\begin{aligned}
\left|L_{x}\left(\xi_{1} \mid \phi(x)-f(x)\right)-L_{x}\left(\xi_{2} \mid \phi(x)-f(x)\right)\right|^{2} & \\
& \leqq \frac{|\lambda|^{4}}{\beta^{2}} \int_{a}^{b}|f(x)|^{2} d x G\left(\xi_{1}, \xi_{2}\right)
\end{aligned}
$$

(for $\xi_{1}, \xi_{2}$ on $\Gamma$ ); thus, under (6.5) and (6.5a), for every solution $\phi(x)$, included in $L_{2}$, of (6.2) the function

$$
L_{x}(\xi \mid \phi(x)-f(x))
$$

will be continuous in $\xi$ for $\xi$ on $\Gamma(\beta \neq 0)$.

When $\phi^{\delta_{0}}(x)$ satisfies (6.1), in consequence of $(6.1 \mathrm{a})$ one has

$$
\begin{gathered}
\left|L_{x}\left(\xi \mid \phi^{\delta_{0}}(x)\right)\right| \leqq|\lambda|\left[\int_{a}^{b}\left|L_{x}\left(\xi \mid K^{\delta_{0}, r}(x, y)\right)\right|^{2} d y\right]^{1 / 2}\left[\int_{a}^{b}\left|\phi^{\delta_{0}}(x)\right|^{2} d x\right]^{1 / 2} \\
+\left|L_{x}(\xi \mid f(x))\right|
\end{gathered}
$$


whence, by (3.19a) for $\left(\phi^{\delta_{0}}(x)\right)$ and (2.21) (for $\left.n=1\right)$,

$$
\begin{aligned}
\left|L_{x}\left(\xi \mid \phi^{\delta_{0}}(x)\right)\right| \leqq & \frac{|\lambda|^{2}}{|\beta|}\left[\int_{a}^{b}|f(x)|^{2} d x\right]^{1 / 2}\left[\int_{a}^{b} \gamma^{2}(\xi \mid y) d y\right]^{1 / 2} \\
& +\left|L_{x}(\xi \mid f(x))\right| .
\end{aligned}
$$

Similarly

$$
\left|L_{x}\left(\xi \mid \phi^{\delta_{0}}(x)-f(x)\right)\right|^{2} \leqq \frac{|\lambda|^{4}}{\beta^{2}} \int_{a}^{b}|f(x)|^{2} d x \int_{a}^{b} \gamma^{2}(\xi \mid y) d y .
$$

On the other hand, by (6.1a) (for $\xi_{1}$ and $\xi_{2}$ ) we obtain the inequality subsequent to $(6.5 \mathrm{~b})$, with $\phi(x)$ and $K(x, y)$ replaced by $\phi^{\delta_{0}}(x)$ and $K^{\delta_{0}}(x, y)$, respectively; an application of (3.19a) (for $\phi^{\delta_{0}}(x)$ ) will yield

$$
\begin{aligned}
\mid L_{x}\left(\xi_{1} \mid \phi^{\delta_{0}}(x)-f(x)\right)-L_{x}\left(\xi_{2} \mid \phi^{\delta_{0}}(x)\right. & -f(x))\left.\right|^{2} \\
& \leqq \frac{|\lambda|^{4}}{\beta^{2}} \int_{a}^{b}|f(x)|^{2} d x G\left(\xi_{1}, \xi_{2}\right),
\end{aligned}
$$

(for $\xi_{1}, \xi_{2}$ on $\Gamma$ ) if $L$ is such that (6.5) holds.

If (6.5) and (6.5a) are assumed, in view of (6.6b) and of (6.7) application of Vitali's theorem (on limits of analytic functions) is possible in a manner analogous to that in (C, p. 55). The following result is obtained.

Let $K(x, y) \subset H_{1}$ and $L$ be an "associated" operator (Definition 2.3), satisfying (6.5), (6.5a) and let $\Lambda=\beta \neq 0$. For a suitable choice of $\delta_{0, r}$ not only will $\phi^{\delta_{0}, r}(x)$ converge (weakly) to a solution $\phi(x)$ satisfying (6.2) but the function $L_{x}(\xi \mid \phi(x)-f(x))$ will be continuous in $\xi($ for $\xi$ in $\Gamma)$ and will be regular in $\lambda$ for all non-real $\lambda$ (when $\xi$ is in $\Gamma$ ).

The analogue of $\left(\mathrm{C}\right.$, Theorems $\left.\mathrm{III}_{2}, \mathrm{IV}_{2}\right)$, for kernels $K(x, y) \subset H_{1}$ and having "associated" with them an operator $L$, is obtained by passage to the limit. The result reads as follows. Given a value $\lambda=\lambda_{0}\left(\lambda_{0}=\alpha_{0}+i \beta_{0} ; \beta_{0} \neq 0\right)$, there exists an operator $T_{0}$ (depending on $\lambda_{0}$, but independent of $f$ ) so that

$$
\phi(x)=T_{0}(f(x))
$$

will constitute a solution of (6.2); moreover,

$$
\int_{a}^{b} T_{0}\left(f_{2}(x)\right) \cdot f_{1}(x) d x=\int_{a}^{b} T_{0}\left(f_{1}(x)\right) \cdot f_{2}(x) d x,
$$

whenever $f_{1}(x), f_{2}(x) \subset L_{2}$. In particular, if for $\lambda=\lambda_{0}$ the equation (6.2) has only one solution $\phi(x) \subset L_{2},(6.8)$, (6.8a) will hold.

The analogue to $\left(\mathrm{C}\right.$, Theorem $\left.\mathrm{II}_{2}\right)$ will be as follows. 
If the operator $L$ is such that $L_{x}(\xi \mid q(x))$ is real for $q(x)$ real and the

$$
\text { conjugate of } L_{x}(\xi \mid q(x))=L_{x}(\xi \mid \bar{q}(x)) \text {, }
$$

and if for a particular $\lambda_{0}\left(I \lambda_{0}=\beta_{0} \neq 0\right)$ the homogeneous equation (6.2a) has no solutions included in $L_{2}$, except $\phi(x)=0$ (almost everywhere), then for all non-real values of $\lambda(6.2 \mathrm{a})$ will have no solutions included in $L_{2}$, except zero (almost everywhere).

The proof of this theorem is closely analogous to that of $\left(\mathrm{C}\right.$, Theorem $\left.\mathrm{II}_{2}\right)$. However, in view of the extension, to be given in the sequel, of this result to classes $H_{n}$ it is desirable to outline briefly a sketch of the proof.

If the theorem is not true, then

$$
L_{x}(\xi \mid \phi(x))-\lambda_{0} \int_{a}^{b} L_{x}(\xi \mid K(x, y)) \phi(y) d y=L_{x}\left(\xi \mid\left(1-\lambda_{0} / \lambda\right) \phi(x)\right),
$$

where $\phi(x) \subset L_{2}, \phi(x) \neq 0$, and $\phi(x)$ is a solution of (6.2a) for a value $\lambda$ $(\Lambda=\beta \neq 0)$. Using (6.9) one obtains

(6.10a) $L_{x}\left(\xi \mid \phi_{1}(x)\right)-\lambda_{0} \int_{a}^{b} L_{x}(\xi \mid K(x, y)) \phi_{1}(y) d y=L_{x}\left(\xi \mid\left(1-\lambda_{0} / \lambda^{\prime}\right) \phi_{1}(x)\right)$.

In consequence of the statement in connection with (6.8) and (6.8a),

$$
\left(1-\frac{\lambda_{0}}{\lambda^{\prime}}\right) \int_{a}^{b}|\phi(x)|^{2} d x=\left(1-\frac{\lambda_{0}}{\lambda}\right) \int_{a}^{b}|\phi(x)|^{2} d x,
$$

and, inasmuch as $\int|\phi|^{2} d x \neq 0$, necessarily $\beta=0$ which is contrary to hypothesis.

Similarly, following the lines indicated in (C) one may prove the following analogue to the important result of $\left(\mathrm{C}\right.$, Theorem $\left.\mathrm{V}_{2}\right)$.

If the operator $L$ (associated with $K(x, y) \subset H_{1}$ ) satisfies the condition (6.9), then the number of linearly independent solutions [included in $L_{2}$ ] of the homogeneous equation (6.2a) is the same for all $\lambda(\Lambda=\beta \neq 0)$.

With respect to linear independence of solutions of (6.2a) the following will hold.

Let $\phi_{1}(x), \cdots, \phi_{n}(x)$ be solutions included in $L_{2}$ of the homogeneous equation (6.2a), corresponding to the distinct values of $\lambda$,

$$
\lambda_{1}, \cdots, \lambda_{n} \quad\left(\lambda_{\nu} \neq 0 ; \nu=1, \cdots, n\right) ;
$$

the $\phi_{j}(x)$ will be linearly independent if $L$ ("associated" with $K(x, y)$ ) is such that

$$
L_{x}(\xi \mid q(x))=0
$$$$
\left(q(x) \subset L_{2}\right)
$$

implies that $q(x)=0$ (almost everywhere). 
In fact, if this theorem is not true, then for some $c_{\nu}$

$$
c_{1} \phi_{1}(y)+\cdots+c_{n} \phi_{n}(y)=0 \quad\left(\text { not all } c_{\nu}=0\right) .
$$

Multiplying by $L_{x}(\xi \mid K(x, y)) d y$, integrating and making use of

$$
L_{x}\left(\xi \mid \phi_{\nu}(x)\right)=\lambda_{\nu} \int_{a}^{b} L_{x}(\xi \mid K(x, y)) \phi_{\nu}(y) d y,
$$

we obtain

$$
\sum_{\nu=1}^{n} \frac{c_{\nu}}{\lambda_{\nu}} L_{x}\left(\xi \mid \phi_{\nu}(x)\right)=L_{x}\left(\xi \mid \sum_{\nu=1}^{n} \frac{c_{\nu}}{\lambda_{\nu}} \phi_{\nu}(x)\right)=0 .
$$

In view of the property in connection with (6.12),

$$
\sum_{\nu=1}^{n} \frac{c_{\nu}}{\lambda_{\nu}} \phi_{\nu}(x)=0
$$

Repeating this process a number of times and at each step making use of (6.13) and of the property referred to above, a set of equations is obtained which cannot be satisfied, unless all the $c_{\nu}$ are zero.

Let us examine now the question of the range of values which, for $x$ and $\lambda=\lambda_{1}\left(\beta_{1} \neq 0\right)$ fixed, could be assumed by the solutions of (6.2).*

According to the italicized statement subsequent to (6.11) the number of linearly independent solutions of $(6.2 \mathrm{a})$ (where $K(x, y) \subset H_{1}$ and $L$ is an associated operator) is the same for all non-real $\lambda$. It can be arranged to have these solutions forming an orthogonal and normal set (for a fixed $\lambda$ ). Let $\Phi_{1}(x), \Phi_{2}(x), \cdots$ constitute a full set of such description for $\lambda_{1}$. Let $\phi(x)$ be any solution of (6.2) for $\lambda_{1}$, the corresponding $\psi(x)$ being defined (cf. (6.3)) by

$$
\phi(x)=\frac{i \lambda_{1}^{\prime}}{2 \beta_{1}}(f(x)+\psi(x))\left(\lambda_{1}^{\prime}=\text { conjugate of } \lambda_{1}\right) .
$$

Then the result of the same form as given in $(\mathrm{C}, \mathrm{pp} .71,72)$ will hold for equations (6.2):

$$
\begin{aligned}
& \left|\phi(x)-c_{r}\left(x, \lambda_{1}\right)\right| \leqq r\left(x, \lambda_{1}\right) \quad\left[c\left(x, \lambda_{1}\right)=\left(i \lambda_{1}^{\prime} / 2 \beta_{1}\right)(f(x)+w(x))\right] \\
& r^{2}\left(x, \lambda_{1}\right)=\frac{\left|\lambda_{1}\right|^{2}}{4 \beta_{1}^{2}} \int_{a}^{b}\left[|f(t)|^{2}-|w(t)|^{2}\right] d t \sum_{\nu}\left|\Phi_{\nu}(x)\right|^{2}
\end{aligned}
$$

where $w(x)$ is from the Fourier-expansion (in terms of the $\Phi_{\nu}(x)$ ) of $\psi(x)$,

$$
\psi(x)=w(x)+\sum c_{\nu} \Phi_{\nu}(x)
$$
tors $L$.

* A problem of this type is treated in (C) for kernels not of class $H_{1}$ and without the aid of opera- 
(cf. (6.14)), and is independent of $\phi(x) .{ }^{*}$ To establish this one needs only to take note of the inequality (6.3b) and to follow the procedure indicated in (C).

Corresponding to the function $w(x)$ (of $(6.15 \mathrm{~b})$ ) there is a particular solution (for $\lambda_{1} ; \beta \neq 0$ ) of (6.2),

$$
\phi_{0}(x)=\frac{i \lambda_{1}}{2 \beta_{1}}(f(x)+w(x)) .
$$

In view of the statement in connection with (6.4), from (6.15) and (6.15a) it is inferred that, if there exists a sequence $\left\{\phi^{\delta_{0}, r}(x)\right\} \dagger$ which converges in the weak sense to $\phi_{0}(x)$, while

$$
\lim \int_{a}^{b}\left|\phi^{\delta_{0}, r}(x)\right|^{2} d x=\int_{a}^{b}\left|\phi_{0}(x)\right|^{2} d x
$$

(cf. (6.16)), then $\phi_{0}(x)$ is the only solution (for $\left.\lambda_{1}\right)$ included in $L_{2}$ of (6.2).

Consider a value $\lambda=\lambda_{1}$ (with $\beta_{1} \neq 0$ ). If not every solution of the homogeneous equation (6.2a), for $\lambda=\lambda_{1}$ is zero (almost everywhere), then the number $r\left(x, \lambda_{1}\right)$, involved in (6.15a), will be distinct from zero at least for some $f(x) \subset L_{2}$, provided the operator $L$ satisfies the condition (6.9). In fact, with the aid of the latter condition the procedure given in (C) (for the demonstration of an analogous result) is applicable, leading to the stated assertion.

7. Extension of the results of $\S 6$ to the classes $H_{n}$. With a view to proof by induction let us assume that the following holds for kernels $K(x, y)$ of classes $H_{n}(n=1,2, \cdots, m-1)$, it being understood that "associated" with every kernel $K(x, y)$, under consideration, there is an operator $L$ (Definition 2.3). For convenience we collect the requisite equations:

$$
\begin{gathered}
\phi^{\delta_{0}} \cdots, \delta_{n-1}(x)-\lambda \int_{a}^{b} K^{\delta_{0}} \cdots, \delta_{n-1}(x, y) \phi^{\delta_{0}}, \cdots, \delta_{n-1}(y) d y=f(x), \\
L_{x}\left(\xi \mid \phi^{\delta_{0}}, \cdots, \delta_{n-1}(x)\right)-\lambda \int_{a}^{b} L_{x}\left(\xi \mid K^{\delta_{0}}, \cdots, \delta_{n-1}(x, y)\right) \phi^{\delta_{0}}, \cdots, \delta_{n-1}(y) d y \\
=L_{x}(\xi \mid f(x)), \\
L_{x}(\xi \mid \phi(x))-\lambda \int_{a}^{b} L_{x}(\xi \mid K(x, y)) \phi(y) d y=L_{x}(\xi \mid f(x)), \\
L_{x}(\xi \mid \phi(x))-\lambda \int_{a}^{b} L_{x}(\xi \mid K(x, y)) \phi(y) d y=0 .
\end{gathered}
$$

If a solution $\phi(x)$ (for a value $\lambda$, with $\beta \neq 0$ ) of (7.2) is the repeated limit in the weak sense of solutions of (7.1) (cf. (3.19)), then

* $w(x)$ is the analogue of $\psi_{0}(x)$ of (C, p. 71); that is, $w(x)$ minimizes $\int(\psi)^{2} d x$ (for $\phi(x)$ satisfying (6.2)). Thus, $w(x)$ is a particular function $\psi(x)$. Obviously $\int w \bar{\Phi}_{\nu} d x=0$.

$\dagger \phi^{\delta_{0}, r(x)}$ a solution of (6.1) (for $\left.\delta_{0}=\delta_{0, r}\right)$. 


$$
\int_{a}^{b}|\psi(x)|^{2} d x \leqq \int_{a}^{b}|f(x)|^{2} d x \quad\left[\phi(x)=\left(i \lambda^{\prime} / 2 \beta\right)(f(x)+\psi(x))\right] .
$$

The necessary and sufficient condition under which (7.3) holds with the equality sign is that

$$
\lim _{\delta_{0, r}} \lim _{\delta_{1, r}} \cdots \lim _{\delta_{n-1, r}} \int_{a}^{b}\left|\phi^{\delta_{0}, r, \delta_{1}, r}, \cdots, \delta_{n-1, r}(x)\right|^{2} d x=\int_{a}^{b}|\phi(x)|^{2} d x
$$

(the $\delta_{\nu, r}$ from (3.19)).

If the operator $L$ ("associated" with $K(x, y)$ ) is defined for $\xi$ in a set $\Gamma$, dense in itself, and if

$$
\begin{array}{r}
\int_{a}^{b}\left|L_{x}\left(\xi_{1} \mid K^{\delta_{0}} \cdots, \delta_{n-1}(x, y)\right)-L_{x}\left(\xi_{2} \mid K^{\delta_{0}} \cdots, \delta_{n-1}(x, y)\right)\right|^{2} d y \leqq G\left(\xi_{1}, \xi_{2}\right) \\
{\left[G\left(\xi_{1}, \xi_{2}\right) \rightarrow 0 \text { as } \xi_{1}-\xi_{2} \rightarrow 0 ; \xi_{1}, \xi_{2} \text { in } \Gamma\right],}
\end{array}
$$

then (7.5) will hold also for $K(x, y)$.

Every solution $\phi(x) \subset L_{2}$ of (7.2) is such that

$$
L_{x}(\xi \mid \phi(x)-f(x))
$$

is continuous in $\xi$ for $\xi$ in $\Gamma$, provided (7.5) holds.

Let a solution $\phi(x)$ of (7.2) be defined by the repeated limiting process of (3.19). If (7.5) holds, then with a suitable choice of the $\delta_{\nu, r}$,

$$
L_{x}(\xi \mid \phi(x)-f(x))
$$

will be continuous in $\xi$, for $\xi$ in $\Gamma$, and will be regular in $\lambda$ for all non-real $\lambda$ $(\xi$ in $\Gamma)$.

(1) The statement in connection with (6.8), (6.8a) holds with respect to the nonhomogeneous equation (7.2) for kernels of classes $H_{\nu}(\nu<m)$.

Also the following holds.

(2) If $L_{x}(\xi \mid q(x)$ ) is real for $q(x)$ real and (6.9) holds and if the equation (7.2a) has no solutions included in $L_{2}$, except zero, then the same will be true for all non-real values of $\lambda$; the number of linearly independent solutions, included in $L_{2}$, of $(7.2 \mathrm{a})$ is the same for all non-real $\lambda$.

(3) Regarding linear independence we have the result, previously stated in connection with (6.12), holding for classes $H_{1}, \cdots, H_{m-1}$.

(4) The result stated in connection with (6.14)-(6.15b) holds for $H_{n}(n<m)$.

If $\phi_{0}(x)$ is a solution for $\lambda_{1}\left(\beta_{1} \neq 0\right)$ of (7.2), which is a repeated limit (in the weak sense) as indicated in (3.19) and which is such that

$$
w(x)=\left(2 \beta_{1} / i \lambda_{1}^{\prime}\right)\left(\phi_{0}(x)-f(x)\right)
$$


renders

$$
\int_{a}^{b}|\psi(x)|^{2} d x
$$

$\left[\phi(x)=\left(i \lambda_{1}{ }^{\prime} / 2 \beta_{1}\right)(f(x)+\psi(x))\right.$ solutions for $\lambda_{1}$ of (7.2)] minimum, then $\phi_{0}(x)$ is the only solution included in $L_{2}$ of (7.2), provided (7.4) holds (with $\left.\phi(x)=\phi_{0}(x)\right)$.

(5) The italicized statement at the end of $\S 6$ holds with respect to the homogeneous equation (7.2a) for classes $H_{1}, \cdots, H_{m-1}$.

All of the above properties have been verified in $\$ 6$ for kernels of class $H_{1}$ (with "associated" operators $L$ ). We shall now establish these properties for $H_{m}$. Let $K^{1}(x, y) \subset H_{m}$ and let $L^{1}$ be an "associated" operator. Then $K^{1 \delta_{0}}(x, y) \subset H_{m-1}$; moreover, as indicated at the beginning of $\$ 5, L^{1}$ will be also associated with $K^{1 \delta_{0}}\left(x_{1}\right)$.

By (7.3), applied to $K^{1 \delta_{0}}(x, y)$,

$$
\int_{a}^{b}\left|\psi^{\delta_{0}}(x)\right|^{2} d x \leqq \int_{a}^{b}|f(x)|^{2} d x .
$$

With $\phi^{1}(x)=\lim \phi^{\delta_{0}, r}(x)$ (in the weak sense) we shall have $\psi^{1}(x)=\lim \psi^{\delta_{0}, r}(x)$ (in the weak sense), where

$$
\phi^{\delta_{0}}(x)=i \lambda^{\prime} / 2 \beta\left(f(x)+\psi^{\delta_{0}}(x)\right), \quad \phi^{\prime}(x)=i \lambda^{\prime} / 2 \beta\left(f(x)+\psi^{1}(x)\right) .
$$

In view of the theorem of Riesz, stated subsequent to $(6.4 \mathrm{~b})$,

$$
\lim \sup \int_{a}^{b}\left|\psi^{\delta_{0}, r}(x)\right|^{\dot{2}} d x \geqq \int_{a}^{b}\left|\psi^{1}(x)\right|^{2} d x .
$$

By (7.7) and (7.7a)

$$
\int_{a}^{b}\left|\psi^{1}(x)\right|^{2} d x \leqq \int_{a}^{b}|f(x)|^{2} d x,
$$

which is (7.3) for the class $H_{m}$.

Since $K^{\delta_{0}}, \cdots, \delta_{m-1}(x, y) \subset L_{2}$ (in $\left.x, y\right)$ the identity (6.3c) (for $\left.\phi^{\delta_{0}, \cdots, \delta_{m-1}}(x)\right)$ will hold:

$$
\begin{aligned}
\frac{-\beta}{|\lambda|^{2}} \int_{a}^{b}\left|\phi^{\delta_{0}}, \cdots, \delta_{m-1}(x)\right|^{2} d x= & \frac{1}{2 i \lambda} \int_{a}^{b} f(x) \phi_{1}^{\delta_{0}}, \cdots, \delta_{m-1}(x) d x \\
& -\frac{1}{2 i \lambda^{\prime}} \int_{a}^{b} \bar{f}(x) \phi^{\delta_{0}}, \cdots, \delta_{m-1}(x) d x .
\end{aligned}
$$

Suppose (7.4) holds for $\phi^{1}$; thus 
(7.9) $\quad \lim _{\delta_{0}, r} \lim _{\delta_{1, r}} \cdots \lim _{\delta_{m-1, r}} \int_{a}^{b}\left|\phi^{\delta_{0}, r}, \cdots, \delta_{m-1, r}(x)\right|^{2} d x=\int_{a}^{b}\left|\phi^{1}(x)\right|^{2} d x$.

Now (cf. (3.19) for $\phi^{1}$ )

$$
\begin{aligned}
\lim _{\delta_{m-1, r}} \phi^{\delta_{0}, r}, \cdots, \delta_{m-1, r}(x) & =\phi^{\delta_{0, r}} \cdots, \delta_{m-2, r}(x), \\
\lim _{\delta_{m-2, r}} \phi^{\delta_{0}, r}, \cdots, \delta_{m-2, r}(x) & =\phi^{\delta_{0}, r} \cdots, \delta_{m-3, r}(x), \cdots, \lim _{\delta_{0, r}} \phi^{\delta_{0}, r}(x)=\phi^{1}(x)
\end{aligned}
$$

[in the sense of weak convergence], with the functions involved included in $L_{2}$; hence repeated application of Theorem 1.4 to the second member of (7.8) is possible yielding the result (when $\delta_{\nu}=\delta_{\nu, r}$ )

$$
\begin{array}{r}
\lim _{\delta_{0, r}} \cdots \lim _{\delta_{m-1}, r}\left[\frac{1}{2 i \lambda} \int_{a}^{b} f(x) \phi_{1}^{\delta_{0}}, \cdots, \delta_{m-1}(x) d x-\frac{1}{2 i \lambda} \int_{a}^{b} \bar{f}(x) \phi^{\delta_{0}}, \cdots, \delta_{m-1}(x) d x\right] \\
=\frac{1}{2 i \lambda} \int_{a}^{b} f(x) \phi^{1}(x) d x-\frac{1}{2 i \lambda^{\prime}} \int_{a}^{b} \bar{f}(x) \phi^{1}(x) d x .
\end{array}
$$

This, together with (7.9) implies

$$
\frac{-\beta}{|\lambda|^{2}} \int_{a}^{b}\left|\phi^{1}(x)\right|^{2} d x=\frac{1}{2 i \lambda} \int_{a}^{b} f(x) \phi_{1}^{1}(x) d x-\frac{1}{2 i \lambda^{\prime}} \int_{a}^{b} \bar{f}(x) \phi^{1}(x) d x .
$$

Substituting in (7.9a) $\phi^{1}(x)$ in terms of $\psi^{1}(x)$ we obtain the equality

$$
\int_{a}^{b}\left|\psi^{1}(x)\right|^{2} d x=\int_{a}^{b}|f(x)|^{2} d x
$$

which is observed to be a consequence of (7.9). Suppose now that (7.9) does not hold. Since the repeated limit displayed preceding (7.9a) exists even if (7.9) does not hold, in consequence of (7.8) it can be asserted that

$$
\begin{aligned}
\lim _{\delta_{0, r}} \because \lim _{\delta_{m-1, r}} & \int_{a}^{b}\left|\phi^{\delta_{0}, r}, \cdots, \delta_{0, m-1}(x)\right|^{2} d x=\gamma \\
& =\left[\frac{1}{2 i \lambda} \int_{a}^{b} f(x) \phi^{1}(x) d x-\frac{1}{2 i \lambda^{\prime}} \int_{a}^{b} \bar{f}(x) \phi^{1}(x) d x\right] \frac{|\lambda|^{2}}{-\beta} .
\end{aligned}
$$

By the theorem of Riesz (text subsequent to (6.4b)), (7.10) will imply

$$
\gamma \geqq \int_{a}^{b}\left|\phi^{1}(x)\right|^{2} d x .
$$

This, in view of our previous assumption that (7.9) does not hold, yields the inequality

$$
\gamma>\int_{a}^{b}\left|\phi^{1}(x)\right|^{2} d x .
$$


Substituting in (7.10a) the expression for $\gamma$ from the last member of (7.10) and replacing $\phi^{1}(x)$ in terms of $\psi^{1}(x)$, we infer that failure of (7.9) to hold implies

$$
\int_{a}^{b}\left|\psi^{1}(x)\right|^{2} d x<\int_{a}^{b}|f(x)|^{2} d x .
$$

Accordingly, the statement with respect to (7.4) holds for the class $H_{m}$.

The property stated with reference to $(7.5)$ will hold for $K^{1}(x, y)$ and $L^{1}$ in consequence of (2.22), of (2.21) (applied to the kernel in question) and of Lebesgue's theorem on passage to the limit under the integral sign (we $\operatorname{keep} \beta \neq 0$ ).

Under (7.5) (for $K^{1 \delta_{0}, \cdots, \delta_{m-1}}$ and $L^{1}$ ) the function $L_{x}^{1}\left(\xi \mid \phi^{1}(x)-f(x)\right.$ ), where $\phi^{1}(x)$ is any solution included in $L_{2}$ of (7.2) [that is, of (7.2) with $L^{1}$ and $K^{1}$ ], will be continuous in $\xi(\xi$ in $\Gamma$ ). In fact, by the same method as used before we obtain the inequality (6.6) for $L^{1}$ and $\phi^{1}$, which justifies the above assertion.

If $\phi^{1}(x)$ is a repeated limit in accordance with (3.19), then

$$
\phi^{\delta_{0}, r}(x) \rightarrow \phi^{1}(x) \quad \text { (weakly), }
$$

where

$$
\phi^{\delta_{0}, r}(x)=\lim _{\delta_{1, r}} \cdots \lim _{\delta_{m-1, r}} \phi^{\delta_{0, r}, \delta_{1, r}} \cdots, \delta_{m-1, r}(x) \quad \text { (weakly), }
$$

and $\phi^{\delta_{0}, r}(x)$ satisfies

$$
\text { (7.11a) } L_{x}^{1}\left(\xi \mid \phi^{\delta_{0}, r}(x)\right)-\lambda \int_{a}^{b} L_{x}^{1}\left(\xi \mid K^{\delta_{0}, r}(x, y)\right) \phi^{\delta_{0}, r}(y) d y=L_{x}^{1}(\xi \mid f(x)) \text {, }
$$

with $K^{\delta_{0}, r}$ formed corresponding to $K^{1}(x, y)$. Proceeding with respect to (7.11a) as before one obtains the inequalities (6.6b), (6.6c), (6.7) (for $L^{1}$ ); the latter inequality will hold under $(7.5)$ (for $\left.L^{1}, K^{1 \delta_{0}}, \cdots, \delta_{m-1}\right)$. With the aid of Vitali's theorem these inequalities enable us to assert that the statements made with respect to (7.5c) hold for $L^{1}$ and $\phi^{1}$, as well.

We shall now extend the property (1) to $L^{1}$ and $K^{1}$ (cf. (6.8), (6.8a)). Now in (7.11a) $K^{\delta_{0}, r}(x, y) \subset H_{m-1}$; thus, by hypothesis, given any $\lambda=\lambda_{0}=\alpha_{0}+i \beta_{0}\left(\beta_{0} \neq 0\right)$, there exists an operator $T_{0}^{\delta_{0}, r}$ (depending on $\lambda_{0}$, independent of $f$ ) so that $\phi^{\delta_{0}, r}(x)=T_{0}^{\delta_{0}, r}(f(x)) \subset L_{2}$ will be a solution of (7.11a) for all $f(x) \subset L_{2}$ and so that

$$
\int_{a}^{b} T_{0}^{\delta_{0}, r}\left(f_{2}(x)\right) f_{1}(x) d x=\int_{a}^{b} T_{0}^{\delta_{0}, r}\left(f_{1}(x)\right) f_{2}(x) d x \quad\left(\text { for } f_{1}, f_{2} \subset L_{2}\right) .
$$

The $\delta_{0, r}$ can be so chosen that

$$
T_{0}^{\delta_{0}, r}(f(x)) \rightarrow \phi^{1}(x) \quad \text { (in the weak sense), }
$$


where $\phi^{1}(x) \subset L_{2}$ constitutes a solution of (7.2) (for $K^{1}, L^{1}, \lambda_{0}$ ), and so that the relationship (7.12a) holds for all $f(x) \subset L_{2}$. The function $\phi^{1}(x)$ of (7.12a) will then be related to an operation $T_{0}^{1}$,

$$
T_{0}^{1}(f(x))=\phi^{1}(x)
$$

$\left(T_{0}{ }^{1}\right.$ independent of $\left.f\right)$,

defined for all $f(x) \subset L_{2}$. By Theorem 1.4 and since $T_{0}^{\delta_{0}, r}(f(x)) \subset L_{2}$ and (7.12a), (7.12b) hold, from (7.12) by passing to the limit it is inferred that

$$
\int_{a}^{b} T_{0}^{1}\left(f_{2}(x)\right) f_{1}(x) d x=\int_{a}^{b} T_{0}^{1}\left(f_{1}(x)\right) f_{2}(x) d x,
$$

whenever $f_{1}, f_{2} \subset L$. The extension of (1) to the class $H_{m}$ is immediate.

To establish the first part of (2) we assume (6.9) (for $L^{1}$ ) and, using (7.12c), repeat the steps (6.10)-(6.11) with reference to $L^{1}$ and $K^{1}$. It remains to demonstrate that the number of linearly independent solutions, included in $L_{2}$, of (7.2a) (for $L^{1}, K^{1}$ ) is the same for all non-real $\lambda$ (under (6.9) for $L^{1}$ ). This is inferred with the aid of the operator $T_{0}{ }^{1}$ of $(7.12 \mathrm{~b})$, using arguments of the type given in $\left(\mathrm{C}\right.$, proof of Theorem $\left.\mathrm{V}_{5}\right)$.

The property (3) (for $L^{1}, K^{1}$ ) is established as in the text in connection with (6.12)-(6.13a).

The statement (4) is extended to the class $H_{m}$ on the basis of the inequality of (7.3), which has been already demonstrated for kernels included in $H_{m}$.

A consequence of this extension is that we are now able to assert that the result stated with regard to (7.6), (7.6a) holds for the class $H_{m}$, as well.

Similarly, it is seen that (5) will hold for $K^{1}$ and $L^{1}$.

Theorem 7.1. The statements made, from (7.1) to (7.6a) and (5) (inclusive), will hold true, with respect to the equations (7.2), (7.2a), for all classes $H_{n}$ (finiten).

8. Some further results for classes $H_{n}$. The following formulas (cf. (8.1)(8.4)), which were established in (C) for the class $H_{1}$, will hold for all classes $H_{n}$, provided that we envisage only kernels with which one may "associate" (Definition 2.3) operators $L$ and provided that (7.2a) $(I \lambda \neq 0)$ has $\phi(y)=0$ (almost everywhere) as the only solution included in $L_{2}$.

One has

$$
\begin{aligned}
\int_{a}^{b}\left[\frac{\partial}{\partial x} \int_{\Delta_{1}} \mu d_{\mu} \Omega(x, y \mid \mu)\right]\left[\frac{\partial}{\partial x} \Delta_{2} \Omega(x, z \mid \mu)\right] d x \\
=\int_{a}^{b}\left[\frac{\partial}{\partial x} \int_{\Delta_{2}} \mu d_{\mu} \Omega(x, z \mid \mu)\right]\left[\frac{\partial}{\partial x} \Delta_{1} \Omega(x, y \mid \mu)\right] d x . *
\end{aligned}
$$

* If the difference operator $\Delta$ corresponds to the interval $\left(\lambda^{\prime}, \lambda^{\prime \prime}\right)$, integration extended over $\Delta$ will be understood to be between the limits $\lambda^{\prime}, \lambda^{\prime \prime}$. 
When the intervals corresponding to $\Delta_{1}, \Delta_{2}$ are nonoverlapping,

$$
\int_{a}^{b}\left[\frac{\partial}{\partial x} \Delta_{1} \Omega(x, y \mid \lambda)\right]\left[\frac{\partial}{\partial x} \Delta_{2} \Omega(x, z \mid \lambda)\right] d x=0 ;
$$

moreover,

$$
\begin{aligned}
& L_{x}\left(\xi \mid \frac{\partial}{\partial x} \Delta \Omega(x, z \mid \lambda)\right) \\
&=L_{x}\left(\xi \mid \frac{\partial}{\partial x} \int_{a}^{b} \frac{\partial}{\partial y} \Omega(x, y \mid \lambda) \frac{\partial}{\partial y} \Omega(y, z \mid \lambda) d y\right) .
\end{aligned}
$$

When the only solution of $L(\xi \mid \phi(x))=0\left(\phi(x) \subset L_{2}\right)$ is zero,

$$
\Delta \Omega(x, z \mid \lambda)=\int_{a}^{b} \frac{\partial}{\partial y} \Omega(x, y \mid \lambda) \frac{\partial}{\partial y} \Omega(y, z \mid \lambda) d y .
$$

To demonstrate (8.1) one may proceed as follows. By (3.27) the equations

$$
\begin{aligned}
L_{x}\left(\xi \mid \phi_{1}(x)\right)-\lambda \int_{a}^{b} L_{x}(\xi \mid K(x, s)) \phi_{1}(s) d s & \\
& =L_{x}\left(\xi \mid \frac{\partial}{\partial x} \int_{\Delta_{1}}(\mu-\lambda) d_{\mu} \Omega(x, y \mid \mu)\right),
\end{aligned}
$$

$$
\begin{aligned}
L_{x}\left(\xi \mid \phi_{2}(x)\right)-\lambda \int_{a}^{b} L_{x}(\xi \mid K(x, s)) \phi_{2}(s) d s & \\
= & L_{x}\left(\xi \mid \frac{\partial}{\partial x} \int_{\Delta_{2}}(\mu-\lambda) d_{\mu} \Omega(x, z \mid \mu)\right)
\end{aligned}
$$

possess solutions

$$
\phi_{1}(x)=\frac{\partial}{\partial x} \int_{\Delta_{1}} \mu d_{\mu} \Omega(x, y \mid \mu), \quad \phi_{2}(x)=\frac{\partial}{\partial x} \int_{\Delta_{2}} \mu d_{\mu} \Omega(x, z \mid \mu),
$$

respectively. Now, by Theorem 7.1 the result stated in connection with (6.8) and (6.8a) holds for all classes $H_{n}$; thus, on writing

$$
f_{1}(x)=\frac{\partial}{\partial x} \int_{\Delta_{1}}(\mu-\lambda) d_{\mu} \Omega(x, y \mid \mu), \quad f_{2}(x)=\frac{\partial}{\partial x} \int_{\Delta_{2}}(\mu-\lambda) d_{\mu} \Omega(x, z \mid \mu),
$$

it is inferred that

$$
\begin{aligned}
\int_{a}^{b}\left[\frac{\partial}{\partial x}\right. & \left.\int_{\Delta_{1}}(\mu-\lambda) d_{\mu} \Omega(x, y \mid \mu)\right]\left[\frac{\partial}{\partial x} \int_{\Delta_{2}} \mu d_{\mu} \Omega(x, z \mid \mu)\right] d x \\
& =\int_{a}^{b}\left[\frac{\partial}{\partial x} \int_{\Delta_{2}}(\mu-\lambda) d_{\mu} \Omega(x, z \mid \mu)\right]\left[\frac{\partial}{\partial x} \int_{\Delta_{1}} \mu d_{\mu} \Omega(x, y \mid \mu)\right] d x .
\end{aligned}
$$


Finally, (8.1) is obtained if one takes $\lambda=1+i \beta(\beta \neq 0)$ and equates the imaginary parts of the two members in $(8.5 \mathrm{~b})$.

In demonstrating the property (8.2) for any class $H_{n}$ one may follow a method analogous to that indicated in (C) for the class $H_{1}$. We shall not give the details.

The identity (8.3) may be established by induction by consecutive passages to the limit.

The property (8.4) is a consequence of (8.3).

The results (8.1)-(8.4), which have been verified for all classes $H_{n}$ $(n=1,2, \cdots)$ are of interest in themselves as well as with a view to further developments for the case when operators $L$, of a more specialized character than required by Definition 2.3, are available.

9. Regarding reducible sets. In the sequel, throughout, we let $\beta$ denote a number of class I or II.* Let $E$ be a nondense closed set on $(a, b)$ with a denumerable derivative $E^{1}$. Then $E$ will be denumerable and we may write

$$
\begin{aligned}
E & =\left(I_{1}, I_{2}, \cdots\right), \\
E^{1} & =\left(I_{1}^{1}, I_{2}^{1}, \cdots\right) ;
\end{aligned}
$$

moreover, $E$ will be reducible and the derivative of order $\beta$ will be zero,

$$
E^{\beta}=0,
$$

for some $\beta$ of class I or II (we take $\beta$ as the least number so that $(9.1 \mathrm{~b})$ holds).

In $\S \S 2-8$ the case corresponding to $\beta$ of class $I$ has been already considered. This is the reason why our attention will now be confined to the case of $\beta$ (in (9.1b)) of class II. Necessarily $\beta$ will be not a limit number. $\dagger$

We shall need the following result.

Let $G_{1}, G_{2}, \cdots$ be a simply infinite $\ddagger$ sequence of closed sets, each containing the next and each having some points not in the next. Let

$$
G=G_{1} G_{2} \ldots \subset O,
$$

where $O$ is an open set. Then either $G_{1} \subset O$ or there exists a number $j$ so that

$$
G_{\nu} \subset O
$$$$
(\nu=j+1, j+2, \cdots),
$$

while

$$
G_{j} \notin O . \S
$$

* It is to be recalled that the numbers of class I are the ordinals $1,2, \cdots$. The numbers not of the first class, but obtainable by the use of the two Cantor generation principles, are of class II. As usual $\omega$ will denote the first number of class II.

$\dagger$ That is, there will be a number $\beta-1$.

$\ddagger$ An infinite sequence $q_{1}, q_{2}, \cdots, q_{n}, \cdots$ is simply infinite if $n<\omega$.

$\S \Phi$ in (9.2b) signifies that $G_{i}$ has points not in $O$. 
Suppose the above is not true. Then every set $G_{\nu}(\nu=1,2, \cdots)$ has a point $b_{\nu}$ exterior to $O$. The point $b_{1}$ will be in each of the sets $G_{1}, G_{2}, \cdots, G_{n_{1}}$ and will be not in $G_{n_{1}+1}$; in fact, if this were not the case $b_{1}$ would be in $G$ and, by (9.5), it would be in $O$. The point $b_{n_{1}+1}$ (exterior to $O$ ), being in $G_{n_{1}+1}$, will be distinct from $b_{1} ; b_{n_{1}+1}$ will belong to the sets

$$
G_{n_{1}+1}, G_{n_{1}+2}, \cdots, G_{n_{2}} \quad\left[b_{n_{1}+1} \subset G_{n_{2}+1}\right],
$$

since otherwise one would have

$$
b_{n_{1+1}} \subset G_{n_{1}+1} G_{n_{1}+2} \cdots=G \subset O,
$$

which presents a contradiction. Thus, step by step we obtain an infinite sequence of points $\left\{b_{n_{i}+1}\right\}\left(0=n_{0}<n_{1}<\cdots\right)$, which are all distinct and are all exterior to $O$, with the point $b_{n_{i}+1}$ belonging to the finite number of sets

$$
G_{n_{i}+1}, G_{n_{i}+2}, \cdots, G_{n_{i+1}},
$$

and not belonging to $G_{n_{i+1}+1}$ (such a point is obtained for $i=0,1, \cdots$ ). Let (9.3a)

be a subsequence of $\left\{b_{n_{i}+1}\right\}$ such that

$$
\lim _{k} c_{k}=c
$$$$
\left[c_{k}=b_{n^{\prime}+1} ; i^{\prime}=i_{k} ; i_{1}<i_{2}<\cdots\right]
$$

exists.

Now, the points $c_{1}, c_{2}, \cdots$ are all in $G_{n_{i^{\prime}+1}}\left(i^{\prime}=i_{1}\right)$; the latter set being closed, $c$ will be in it. In general, the points $c_{k}, c_{k+1}, \cdots$ will be all in $G_{n_{\boldsymbol{i}^{\prime}+1}}$ $\left(i^{\prime}=i_{k}\right)$ and hence, this set being closed,

$$
c \subset G_{n_{i^{\prime}+1}}
$$$$
\left(i^{\prime}=i_{k}\right) \text {. }
$$

The relation (9.3c) is asserted for $i^{\prime}=i_{1}<i_{2}<\ldots$. Clearly $c$ belongs to every set $G_{\nu}(\nu=1,2, \cdots)$ and thus is a point of $G$; hence included in $O$. The latter set being open there exists a closed interval $\Delta$, containing $c$ in the interior and contained in $O$. In $\Delta$ there will be some points $c_{k}$; that is, some points $b_{\nu}$; this is contrary to the italicized statement preceding (9.3). Whence we deduce the truth of the statement in connection with (9.2)-(9.2b).

Conforming with the notation introduced in $\$ 2$, a set $E$ satisfying (9.1b) (as stated) will be said to belong to $R_{\beta-1}$ (Definition 2.1). By definition of $\beta$ the set $E^{\beta-1}$ will have some points; in view of $(9.1 \mathrm{~b})$ the number of these points will be finite. Thus

(9.4) $\quad E^{\beta-1}=\left(I_{1}^{\beta-1}, I_{2}^{\beta-1}, \cdots, I_{k}^{\beta-1}\right)=\left(s_{1}, s_{2}, \cdots, s_{k}\right) \quad(\beta-1$ of class II $)$.

We may also write 


$$
E^{\alpha}=\left(I_{1}^{\alpha}, I_{2}^{\alpha}, \cdots\right)
$$

The sets $E^{\alpha}(\alpha<\beta-1)$ will be all denumerably infinite.

We form a set $\Delta^{1}\left(\delta_{1}\right)$ of closed intervals

$$
\Delta_{\nu}{ }^{1}\left(\delta_{1}\right)=\left(s_{\nu}-\delta_{1}, s_{\nu}+\delta_{1}\right) \quad\left(\nu=1, \cdots, k ; \delta_{1}>0 ;\right. \text { cf. }
$$

in such a way that they have no points in common and that no end point of them is a point of $E$. Given $\epsilon(>0)$, however small, such a construction can always be effected with

$$
0<\delta_{1}<\epsilon .
$$

This is established using the fact that $E$ is nondense. In fact, without loss of generality one may assume (for the purposes of demonstration) that the $s_{\nu}$ are interior to $(a, b)$ and take $\epsilon$ sufficiently small so that the intervals*

$$
\left(s_{\nu}-\epsilon, s_{\nu}+\epsilon\right)
$$$$
(\nu=1, \cdots, k)
$$

are without common points and are interior to $(a, b)$. In the interval $\left(s_{1}, s_{1}+\epsilon\right)$ we find a subinterval $\left(s_{1}+a_{1}^{1}, s_{1}+b_{1}^{1}\right)$ void of points of $E$; in the interval $\left(s_{1}-b_{1}{ }^{1}, s_{1}-a_{1}{ }^{1}\right)$ we then find a subinterval $\left(s_{1}-b_{1}, s_{1}-a_{1}\right)$ free of points of $E$. We now consider the interval $\left(s_{2}+a_{1}, s_{2}+b_{1}\right)\left[\subset\left(s_{2}, s_{2}+\epsilon\right)\right]$; in it is found another interval without any points of $E$, say $\left(s_{2}+a_{1}{ }^{1}, s_{2}+b_{1}{ }^{1}\right)$. Turning our attention to $\left(s_{2}-b_{1}^{1}, s_{2}-a_{1}^{1}\right)$ we find a subinterval $\left(s_{2}-b_{2}, s_{2}-a_{2}\right)$ free of points of $E$. Clearly, the intervals

$$
\left(s_{\nu}-b_{2}, s_{\nu}-a_{2}\right), \quad\left(s_{\nu}+a_{2}, s_{\nu}+b_{2}\right) \quad(\nu=1,2)
$$

will be void of points of $E$. Continuing in this manner one finally obtains numbers $a_{k}, b_{k}$ so that

$\left(s_{\nu}-b_{k}, s_{\nu}-a_{k}\right) \subset\left(s_{\nu}-\epsilon, s_{\nu}\right),\left(s_{\nu}+a_{k}, s_{\nu}+b_{k}\right) \subset\left(s_{\nu}, s_{\nu}+\epsilon\right)(\nu=1,2, \cdots, k)$, and so that the intervals here displayed in the first members are free of points of $E$. Accordingly, if one takes

$$
a_{k}<\delta_{1}<b_{k},
$$

all of the conditions stated in connection with (9.6), (9.6a) will be satisfied. A choice of the $\delta_{\nu}$, according to the above scheme, will be implied throughout in the sequel.

Inasmuch as $\beta$ is not a limit number, there exists a limit number $\gamma \leqq \beta-1$ so that there exists no limit number $\tau$ for which $\gamma<\tau<\beta$. The sets corresponding to $\beta-1, \beta-2, \cdots, \gamma+1$,

$$
E^{\beta-1}, E^{\beta-2}, \cdots, E^{\gamma+1}, E^{\gamma}
$$

\footnotetext{
* Unless stated otherwise, all the intervals will be supposed to be closed.
} 
can be covered in succession by the $\beta-\gamma$ sets

$$
\Delta^{1}\left(\delta_{1}\right), \Delta^{2}\left(\delta_{2}\right), \cdots, \Delta^{\beta-\gamma-1}\left(\delta_{\beta-\gamma-1}\right), \Delta^{\beta-\gamma}\left(\delta_{\beta-\gamma}\right),
$$

each set $(9.7 \mathrm{a})$ to consist of a finite number of closed intervals, the totality of all intervals, involved in (9.7a), being without common points, no end point of any of these intervals being coincident with any point of $E$. The consecutive sets $(9.7 \mathrm{a})$ are constructed following the procedure described in $\$ 2$. Thus, $\Delta^{2}\left(\delta_{2}\right)$ will consist of the intervals

$$
\Delta_{\nu}^{2}\left(\delta_{2}\right)=\left(s_{\nu}^{\beta-2}-\delta_{2}, s_{\nu}^{\beta-2}+\delta_{2}\right) \quad\left(\nu=1, \cdots, m\left(\delta_{1}\right)\right),
$$

where the $s_{\nu}{ }^{\beta-2}\left(1 \leqq \nu \leqq m\left(\delta_{1}\right)\right)$ are the points of $E^{\beta-2}$ exterior to the set $\Delta^{1}\left(\delta_{1}\right)$. The set $\Delta^{3}\left(\delta_{3}\right)$ will consist of the intervals

$$
\Delta_{\nu}{ }^{3}\left(\delta_{3}\right)=\left(s_{\nu}^{\beta-3}-\delta_{3}, s_{\nu}^{\beta-3}+\delta_{3}\right)
$$

$\left[\nu=1, \cdots, m\left(\delta_{1}, \delta_{2}\right)\right.$; the $s_{\nu}{ }^{\beta-3}$ are points of $E^{\beta-3}$ exterior to the set $\Delta^{1}\left(\delta_{1}\right)$ $\left.+\Delta^{2}\left(\delta_{2}\right)\right]$. The set $\Delta^{\beta-\gamma-1}\left(\delta_{\beta-\gamma-1}\right)$ will consist of the intervals

$$
\begin{array}{r}
\Delta_{\nu}^{\beta-\gamma-1}\left(\delta_{\beta-\gamma-1}\right)=\left(s_{\nu}^{\gamma+1}-\delta_{\beta-\gamma-1}, s_{\nu}^{\gamma+1}+\delta_{\beta-\gamma-1}\right) \\
{\left[\nu=1, \cdots, m\left(\delta_{1}, \delta_{2}, \cdots, \delta_{\beta-\gamma-2}\right)\right] ;}
\end{array}
$$

where the $s_{\nu}{ }^{\gamma+1}$ are points of $E^{\gamma+1}$ exterior to the set $\Delta^{1}\left(\delta_{1}\right)+\cdots+\Delta^{\beta-\gamma-2}\left(\delta_{\beta-\gamma-2}\right)$. Since $E^{\gamma+1}$ is the derivative of $E^{\gamma}$ it is observed that the limiting points of $E^{\gamma}$ are all interior to

$$
\Delta^{1}\left(\delta_{1}\right)+\Delta^{2}\left(\delta_{2}\right)+\cdots+\Delta^{\beta-\gamma-1}\left(\delta_{\beta-\gamma-1}\right) ;
$$

only a finite number of the points of $E^{\gamma}$, say

$$
s_{1}^{\gamma}, \cdots, s_{m 1}^{\gamma} \quad\left(m^{1}=m\left(\delta_{1}, \delta_{2}, \cdots, \delta_{\beta-\gamma-1}\right)\right),
$$

will be in the open set $(a, b)$ minus the set of $(9.7 \mathrm{c})$. Hence the points $(9.7 \mathrm{~d})$ can be covered by the set $\Delta^{\beta-\gamma}\left(\delta_{\beta-\gamma}\right)$, consisting of intervals

$$
\Delta_{\nu}^{\beta-\gamma}\left(\delta_{\beta-\gamma}\right)=\left(s_{\nu}^{\gamma}-\delta_{\beta-\gamma}, s_{\nu}^{\gamma}+\delta_{\beta-\gamma}\right) \quad\left[\nu=1, \cdots, m^{1}(\mathrm{cf} .(9.7 \mathrm{~d}))\right] .
$$

On taking account of the italics subsequent to (9.7a) it is clear that the points belonging to the sets (9.7) are all in the open set

$$
\Gamma\left(\delta_{1}, \delta_{2}, \cdots, \delta_{\beta-\gamma}\right),
$$

obtained by taking the sum of all the intervals involved in (9.7a) and discarding the end points of these intervals.

Suppose now that the limit number $\gamma$, obtained above, is

$$
\gamma=1 \cdot \omega .
$$

In view of the results $(9.2)-(9.2 \mathrm{~b})$, on noting that $E^{\omega} \subset O_{\omega}$, where $O_{\omega}$ is the 
open set (9.7f), it is inferred that either all the sets of the simply infinite sequence*

$$
E, E^{1}, \cdots, E^{n}, \cdots
$$

are in $O_{\omega}$ or there exists a number $j\left[=j\left(\delta_{1}, \delta_{2}, \cdots, \delta_{\beta-\gamma}\right)<\omega\right]$ so that

$$
E^{i+\nu} \subset O_{\omega} \quad(\nu=1,2, \cdots),
$$

while

$$
E^{i} \pitchfork O_{\omega} .
$$

The derivative $E^{i+1}$ of $E^{i}$ being contained in $O_{\omega}$, only a finite number of points of $E^{i}$, say

$$
s_{1}^{j}, \cdots, s_{m^{1}}^{j} \quad\left[m^{1}=m\left(\delta_{1}, \delta_{2}, \cdots, \delta_{\beta-\gamma}\right)\right],
$$

will be exterior to $O_{\omega}$, no point $(9.8 \mathrm{~d})$ being coincident with any end point of the intervals constituting $O_{\omega}$. The points $(9.8 \mathrm{~d})$ can be covered by the set $\Delta^{\beta-\gamma+1}\left(\delta_{\beta-\gamma+1}\right)$, consisting of the intervals

$$
\begin{array}{r}
\Delta_{\nu}^{\beta-\gamma+1}\left(\delta_{\beta-\gamma+1}\right)=\left(s_{\nu}^{j}-\delta_{\beta-\gamma+1}, s_{\nu}^{j}+\delta_{\beta-\gamma+1}\right) \\
\quad\left[\nu=1, \cdots, m^{1} ; m^{1}\right. \text { from (9.8d)] }
\end{array}
$$

in such a way that the intervals in (9.7a), (9.8e) have no points in common, while no point of $E$ is an end point of these intervals. Following the procedure of $\$ 2$ we find that the remaining sets

$$
E^{i-1}, E^{i-2}, \cdots, E^{1}, E^{0}=E
$$

are covered by the sets

$$
\Delta^{\beta-\gamma+2}\left(\delta_{\beta-\gamma+2}\right), \Delta^{\beta-\gamma+3}\left(\delta_{\beta-\gamma+3}\right), \cdots, \Delta^{\beta-\gamma+j}\left(\delta_{\beta-\gamma+j}\right), \Delta^{\beta-\gamma+j+1}\left(\delta_{\beta-\gamma+j+1}\right) .
$$

Each set (9.8e), (9.9) will consist of a finite number of intervals

$$
\begin{gathered}
\Delta_{\nu}^{\beta-\gamma+i}\left(\delta_{\beta-\gamma+i}\right)=\left(s_{\nu}^{j+1-i}-\delta_{\beta-\gamma+i}, s_{\nu}^{j+1-i}+\delta_{\beta-\gamma+i}\right) \\
{\left[\nu=1, \cdots, m^{1} ; m^{1}=m\left(\delta_{1}, \delta_{2}, \cdots, \delta_{\beta-\gamma+i-1}\right) ; i=1,2, \cdots, j+1\right],}
\end{gathered}
$$

where ${s_{\nu}}^{r}$ is in $E^{r}$; moreover, the construction is so effected that the closed intervals (which are finite in number), involved in the sequence of sets

$$
\Delta^{1}\left(\delta_{1}\right), \Delta^{2}\left(\delta_{2}\right), \cdots, \Delta^{\beta-\gamma+j+1}\left(\delta_{\beta-\gamma+j+1}\right),
$$

are all without common points and that no point of $E$ is an end point of these intervals. It is to be noted that the choice of $\delta_{\nu}(>0)(1<\nu \leqq \beta-\gamma+j+1)$ depends on that of $\delta_{1}, \delta_{2}, \cdots, \delta_{\nu-1}$; however, the choice of $\delta_{1}, \delta_{2}, \cdots, \delta_{\nu-1}$ once

* By definition $E^{\omega}=E^{1} E^{2} \cdots E^{n} \cdots(n<\omega)$. 
made, one may take $\delta_{\nu}$ arbitrarily small and thus one may let $\delta_{\nu}$ approach zero through suitable values.

We thus obtained a type of a covering theorem for the set $E$ in the case when the limit number $\gamma$ involved in (9.7f) is of the form $1 \cdot \omega$.

Suppose now that a covering theorem of the above description holds for all sets $E$ for which

$$
\gamma=\eta \omega, \quad 1 \leqq \eta<\alpha,
$$

where $\alpha$ is a number of 1 st or $2 \mathrm{~d}$ class. We wish to establish such a theorem for $\eta=\alpha$.

CASE 1. $\alpha$ is not a limit number. In this case we make use of the fact that the theorem holds for $\alpha-1$.

CASE 2. $\alpha$ is a limit number. In considering Case 1 it is noted that, by hypothesis, there exists a finite number of sets

$$
\Delta^{1}\left(\delta_{1}\right), \Delta^{2}\left(\delta_{2}\right), \cdots, \Delta^{H}\left(\delta_{H}\right),
$$

each consisting of finite number of closed intervals; the totality of these intervals will be without common points; every point of $E$ will be an interior point of one of these intervals; it may occur that some of the subscripts in (9.12) $(1<\nu<H)$ depend on $\delta_{1}, \delta_{2}, \cdots, \delta_{\nu-1}$. The number $\beta$ (cf. (9.1b)) will be, of course, of the form

$$
\beta=(\alpha-1) \omega+q \quad(0<q<\omega) ;
$$

moreover, according to the hypothesis, a covering theorem of the stated type will hold for every $\beta$ ( $\alpha$ fixed) with $q=1,2, \cdots$, where $q<\omega$. It is desired now to obtain such a result when $\beta$ has a value

$$
\beta^{*}=\alpha \omega+p \quad(0<p<\omega) .
$$

With $E^{\beta^{*}}=0, E^{\beta^{*}-1}$ will consist of a finite number of intervals which can be covered by a set $\Delta^{* 1}\left(\delta_{1}^{*}\right)$ of intervals; in succession we construct sets

$$
\Delta^{* 1}\left(\delta_{1}^{*}\right), \Delta^{* 2}\left(\delta_{2}^{*}\right), \cdots ; \Delta^{* p}\left(\delta_{p}^{*}\right),
$$

analogous to the sets $(9.7 \mathrm{a})$, and with similar properties. In particular, the last set in (9.13) will consist of the intervals

$$
\Delta_{\nu}^{* p}\left(\delta_{p}^{*}\right)=\left(s_{\nu}{ }^{\alpha \omega}-\delta_{p}^{*}, s_{\nu}{ }^{\alpha \omega}+\delta_{p}^{*}\right) \quad\left[\nu=1, \cdots, m^{1}\right],
$$

where $m^{1}=m\left(\delta_{1}^{*}, \delta_{2}^{*}, \cdots, \delta_{p-1}^{*}\right)$. The $s_{\nu}^{\alpha \omega}$ in (9.13a) will be points of $E^{\alpha \omega}$; all the other points of $E^{\alpha \omega}$ (an infinity of them) will be interior to the set

$$
\Delta^{* 1}\left(\delta_{1}^{*}\right)+\cdots+\Delta_{\nu}^{*{ }^{p-1}}\left(\delta_{p-1}^{*}\right) .
$$

By definition 
Also, in view of the preceding it is observed that

$$
E^{\alpha \omega} \subset O,
$$

where $O$ is the open set obtained by discarding in

$$
\Delta^{* 1}\left(\delta_{1}^{*}\right)+\cdots+\Delta_{\nu}^{* p}\left(\delta_{p}^{*}\right)
$$

the end points of the intervals involved. On taking note of the result (9.2)(9.2b), as applied with $G_{\nu}=E^{(\alpha-1) \omega+\nu}$, it is accordingly concluded that for some finite $q(>0)$

while

$$
E^{(\alpha-1) \omega+\nu} \subset O \quad(\nu=q, q+1, \cdots),
$$

$$
E^{(\alpha-1) \omega+q-1} \nsubseteq O,
$$

unless $E^{(\alpha-1) \omega+1} \subset O$. It is arranged so that no points of $E^{(\alpha-1) \omega+q-1}$ is a boundary point of $O$. Only a finite number of points of $E^{(\alpha-1) \omega+\alpha-1}$, say

$$
s_{\nu}^{(\alpha-1) \omega+q-1} \quad\left[\nu=1,2, \cdots, m^{1} ; m^{1}=m\left(\delta_{1}^{*}, \cdots, \delta_{p}^{*}\right)\right],
$$

will be exterior to $(9.14 \mathrm{~b}) . \dagger$ If we consider the closed set

$$
G=E \cdot[(a, b)-O],
$$

the consecutive derivatives of $G$ will be

$$
G^{\nu}=E^{\nu} \cdot[(a, b)-O]
$$$$
(\nu=1,2, \cdots,(\alpha-1) \omega+q),
$$

where $G^{(\alpha-1) \omega+q-1}$ consists of the points (9.14c) and, consequently,

$$
G^{\beta}=0
$$$$
(\beta=(\alpha-1) \omega+q) .
$$

In view of the hypothesis made in conjunction with (9.12), applying the statement, just referred to, to the set $G$, we obtain a finite number of sets (9.12) covering $G$, as stated subsequently to (9.12).

Every point of $G$ is interior to $(a, b)-O ; G$ being closed, the sets (9.12) (covering $G$ ) can be replaced by subsets

$$
\Delta_{1}^{1}\left(\delta_{1}\right), \Delta_{1}^{2}\left(\delta_{2}\right), \cdots, \Delta_{1}^{H}\left(\delta_{H}\right),
$$

respectively, obtained by replacing the intervals involved in (9.12), whenever necessary, by suitable subintervals in such a manner that not only the properties (with respect to (9.16)) of (9.12) are maintained but also every closed interval involved in $(9.16)$ is interior to $(a, b)-O$. The finite sequence of sets (cf. (9.13))

$\dagger$ That is, will be interior points of $(a, b)-O$.

$\ddagger$ In the aforesaid statement replace $E$ by $G$ of $(9.15)$. 


$$
\Delta^{* 1}\left(\delta_{1}^{*}\right), \Delta^{* 2}\left(\delta_{2}^{*}\right), \cdots, \Delta^{* p}\left(\delta_{p}^{*}\right), \Delta_{1}^{1}\left(\delta_{1}\right), \Delta_{1}^{2}\left(\delta_{2}\right), \cdots, \Delta_{1}^{H}\left(\delta_{H}\right)
$$

will have all the required covering properties with respect to the set $E$, for which $E^{\beta^{*}}=0$ (cf. (9.12b)). In other words, if Case 1 (introduced subsequent to $(9.11))$ is on hand and if the theorem holds for $\alpha-1$, then it will also hold for $\alpha$ ( $\alpha$ from (9.11)).

We now consider Case 2, when $\alpha$ in (9.11) is a limit number. Thus, it is assumed that every set $E$ with $E^{\beta}=0$, where

$$
\beta=\eta \omega+q \quad(1 \leqq \eta<\alpha ; 1 \leqq q<\omega), \dagger
$$

can be "covered" by a finite number of sets

$$
\Delta^{1}\left(\delta_{1}\right), \Delta^{2}\left(\delta_{2}\right), \cdots, \Delta^{H}\left(\delta_{H}\right),
$$

each consisting of a finite number of intervals. Let $\bar{E}$ be a set, with $\bar{E}^{\beta^{*}}=0$, where

$$
\beta^{*}=\alpha \omega+p
$$$$
(0<p<\omega)
$$

It is observed that $\bar{E}^{\alpha \omega}$ could be considered as the set common to all the sets

We again form a finite number of sets (cf. (9.13)-(9.14a))

$$
\Delta^{* 1}\left(\delta_{1}^{*}\right), \cdots, \Delta^{* p}\left(\delta_{p}^{*}\right),
$$

each consisting of a finite number of intervals; the last set displayed will be of the form (9.13a), where the $s_{\nu}{ }^{\alpha \omega}$ (finite in number) are points of $\bar{E}^{\alpha \omega}$, the other points of $\bar{E}^{\alpha \omega}$ being interior points of $\Delta^{*_{1}}\left(\delta_{1}^{*}\right)+\cdots+\Delta^{*-1}\left(\delta_{p-1}^{*}\right)$. The sets $(9.17 \mathrm{~d})$ "cover" the sets $\bar{E}^{\beta^{*}-1}, \bar{E}^{\beta^{*}-2}, \cdots, \bar{E}^{\alpha \omega}$. We again have

$$
\bar{E}^{\alpha \omega} \subset O,
$$

where $O$ is the open set, obtained by taking the sum of the sets $(9.17 \mathrm{~d})$ and discarding the end points of the intervals involved.

The sequence of sets

$$
\bar{E}^{1 \omega}, \bar{E}^{2 \omega}, \cdots, \bar{E}^{\eta \omega}, \cdots \quad(\eta<\alpha),
$$

even though denumerable, may be not a simply infinite sequence. It is not difficult to see that the set $\bar{E}^{\alpha \omega}$, which is the product of the sets (9.19), could also be considered as the product of the sets of the following simply infinite sequence:

$\dagger$ It is understood that $E^{\beta-1}$ has some points. In (9.17a) the last set contains just a finite number of points of $E$, all the other points of $E$ being in the other sets of (9.17a). 
provided the $\eta_{v}$ are suitably chosen. $\dagger$

With (9.18) and the above in view, the theorem (9.2)-(9.2b) can be applied with $G=\bar{E}^{\alpha \omega}$ and $G_{\nu}=\bar{E}^{\eta_{\nu} \omega}$. Thus there exists a finite number $j$ so that

$$
\bar{E}^{\eta \nu \omega} \subset O \quad(\nu=j+1, j+2, \cdots),
$$

while

$$
\bar{E}^{\eta_{j} \omega} \pitchfork O,
$$

unless $\bar{E}^{\eta_{1} \omega} \subset O$. No points of $\bar{E}^{\eta_{j} \omega}$ will be coincident with any of the boundary points of $O$. Form the set $E_{1}=\bar{E}^{\eta_{j} \omega}[(a, b)-O]$ and write

$$
E=\bar{E}[(a, b)-O]
$$

then

$$
\begin{gathered}
E^{\gamma}=\bar{E}^{\gamma}[(a, b)-O] \quad(\gamma=1,2, \cdots), \\
E^{\eta_{j} \omega}=E_{1} .
\end{gathered}
$$

Now, by (9.20)

$$
E^{\eta_{j+1} \omega}=0 .
$$

Thus, for some $\beta=\eta_{j} \omega+\tau \leqq \eta_{j+1} \omega$ we have

$$
E^{\beta}=E_{1}^{\tau}=0 .
$$

Since $\eta_{j+1}<\alpha$ one has $\beta<\alpha \omega$; hence in (9.21d) $\beta$ is of the form (9.17). Let (9.17a) constitute the set "covering" $E$. By taking suitable subintervals of the intervals constituting the sets $(9.17 \mathrm{a})$ we correspondingly obtain other "covering" sets:

$$
\Delta_{1}^{1}\left(\delta_{1}\right), \Delta_{1}^{2}\left(\delta_{2}\right), \cdots, \Delta_{1}^{H}\left(\delta_{H}\right), \ddagger
$$

having the same properties as $(9.17 \mathrm{a})$ but which at the same time are interior to $(a, b)-O$. The reasoning in this connection is the same as that previously made in connection with (9.16). Adjoining the sequences (9.22), (9.17d), and

$$
\Delta^{* 1}\left(\delta_{1}^{*}\right), \Delta^{* 2}\left(\delta_{2}^{*}\right), \cdots, \Delta^{* p}\left(\delta_{p}^{*}\right), \Delta_{1}{ }^{1}\left(\delta_{1}\right), \cdots, \Delta_{1}{ }^{H}\left(\delta_{H}\right),
$$

we obtain a finite number of sets, each consisting of a finite number of closed intervals, the totality of these intervals possessing no common points, every point of $\bar{E}$ being an interior point of one of the intervals involved; the se-

$\dagger$ Thus, for instance, if $\alpha=\omega^{3}$, one may take $\eta_{\nu}=\nu \omega^{2}$. With $\alpha$ a limit number, $\eta_{\nu}(<\alpha)$ must be chosen so that, given any $\gamma<\alpha$, one may find a value $\nu(\nu<\omega)$ for which $\gamma<\eta_{\nu}<\alpha$.

$\ddagger$ Here the $\delta_{\nu}$ may be different from the original ones. 
quence (9.23) will "cover" the set $\bar{E}$ in the sense previously attributed to the word "cover."

This completes the transfinite induction.

THEOREM 9.1. Let $E$ be a reducible set as stated at the beginning of this section (cf. (9.1)-(9.1b)). The sets

$$
E, E^{1}, E^{2}, \cdots, E^{\beta-1} \quad(\beta \text { of } 1 \text { st or } 2 d \text { class }) \dagger
$$

can always be "covered," in the sense indicated above by a finite number of sets

$$
\Delta^{1}\left(\delta_{1}\right), \Delta^{2}\left(\delta_{2}\right), \cdots, \Delta^{q}\left(\delta_{q}\right) \quad\left(\delta_{1}>0, \cdots, \delta_{q}>0\right),
$$

the set $\Delta^{v}\left(\delta_{v}\right)$ consisting of a finite number of intervals of length $2 \delta_{v}$, no point of $E$ being an end point of any of the intervals involved.

Note. It is observed that in (9.24) the choice of a particular $\delta_{\nu}(\nu>1)$ depends on that of $\delta_{1}, \cdots, \delta_{\nu-1}$; moreover, having chosen $\delta_{1}, \cdots, \delta_{\nu-1}$, we may take the number $\delta$, arbitrarily small. It is also to be noted that the number $q$ in (9.24) may depend on the choice of $\delta_{1}, \delta_{2}, \cdots$.

10. Kernels of transfinite rank. Such kernels will be introduced by means of the following definition.

DeFINITION 10.1. Let $E$ be a closed reducible set on $(a, b)$, as described in the beginning of $\$ 9$, with $E^{\beta}=0$ ( $\beta$ a non-limit number of class II) and $E^{\beta-1}$ consisting of some points (necessarily finite in number). Let $\Delta^{\nu}\left(\delta_{\nu}\right)[\nu=1,2, \cdots, q$ $(<\omega)$ ] be the corresponding covering sets, referred to in Theorem 9.1. A kernel $K(x, y)$ will be said to belong to the class $H_{\beta}$ if, for all "admissible" values $\delta_{v}$ $(>0 ; \nu=1, \cdots, q)$,

(10.1) $\quad K^{\delta_{1}, \delta_{2}, \cdots, \delta_{q}}(x, y) \subset L_{2}$

(in $x, y ;$ for $a \leqq x, y \leqq b)$.

Here

(10.2) $\quad K^{\delta_{1}, \delta_{2}, \cdots, \delta_{q}}(x, y)=0 \quad\left[x\right.$ in $\sum_{v=1}^{q} \Delta^{\nu}\left(\delta_{\nu}\right) ; a \leqq y \leqq b$ (or $\left.\left.a \leqq y<x\right)\right]$;

(10.2a) $\quad K^{\delta_{1}, \delta_{2}, \cdots, \delta_{q}}(x, y)=0 \quad\left[y\right.$ in $\sum_{\nu=1}^{q} \Delta^{\nu}\left(\delta_{\nu}\right) ; a \leqq x \leqq b$ (or $\left.\left.a \leqq x<y\right)\right]$;

(10.2b) $\quad K^{\delta_{1}, \delta_{2}, \cdots, \delta_{q}}(x, y)=K(x, y) \quad$ [at all other points of $\left.a \leqq x, y \leqq b\right]$.

We get in succession

$$
\lim _{\delta_{q}} K^{\delta_{1}, \cdots, \delta_{q}}(x, y)=K^{\delta_{1}} \cdots, \delta_{q-1}(x, y),
$$

(10.3) $\lim _{\delta_{q-1}} K^{\delta_{1}}, \cdots, \delta_{q-1}(x, y)=K^{\delta_{1}} \cdots, \delta_{q-2}(x, y), \cdots$,

$$
\lim _{\delta_{2}} K^{\delta_{1}, \delta_{2}}(x, y)=K^{\delta_{1}}(x, y), \quad \lim _{\delta_{1}} K^{\delta_{1}}(x, y)=K(x, y) .
$$

Thus $K(x, y)$ is a $q$-fold repeated limit of the function of the first member in

$\dagger$ As noted before, necessarily $\beta$ is not a limit number. 
$(10.1) . \dagger$ Here and in the sequel it will be implied that the $\delta_{\nu}$ have "admissible" values $\ddagger$ and that, whenever we let $\delta_{\nu} \rightarrow 0, \delta_{\nu}$ approaches zero through such values.

Unless stated otherwise the number $\beta$, referred to in Definition 10.1, will be taken of the special form

$$
\beta=\omega+p \quad(0<p<\omega ; \omega \text { the first number of class II }) ;
$$

this is in order to gain simplicity of exposition. Most of the facts established for the case of (10.4) could be extended without difficulty to kernels for which $\beta$ is any transfinite number of class II. In this connection the method of transfinite induction, along lines already employed in $\$ 9$, would suffice for demonstration of the majority of the results.

Example of a kernel included in $H_{\omega+1}$. We shall construct a set $E$ with $E^{\omega+1}=0$ and $E^{\omega}$ containing at least one point. Following a device indicated by $\mathrm{H}$. Lebesgue $\S$ we define $E$ as follows. Let $F_{i}$ be the operation such that, if $H$ is a set of points on $(0,1), F_{i}(H)$ is the set of points obtained by a homothetic transformation of $H$ upon the interval $(1 /(i+1), 1 / i) . \|$ Let $O$ represent the point zero. Form in succession the sets

$$
\begin{aligned}
& A_{1}=O+F_{1}(O)+F_{2}(O)+\cdots, \\
& A_{2}=O+F_{1}\left(A_{1}\right)+F_{2}\left(A_{1}\right)+\cdots \\
& \cdot . \cdot \cdot \cdot \cdot \cdot \cdot \cdot \cdot \cdot \cdot \cdot \cdot \cdot, \\
& A_{n}=O+F_{1}\left(A_{n-1}\right)+F_{2}\left(A_{n-1}\right)+\cdots,
\end{aligned}
$$

With the aid of the sets (10.5) one may form the set

$$
E=A_{\omega}=O+F_{1}\left(A_{1}\right)+F_{2}\left(A_{2}\right)+\cdots+F_{m}\left(A_{m}\right)+\cdots ;
$$

$E$ will be reducible and

$$
E^{\omega}=O, \quad E^{\omega+1}=0 .
$$

Let us find the consecutive derivatives of $E$. It is observed that $E^{1}=O+F_{1}^{1}\left(A_{1}\right)+F_{2}^{1}\left(A_{2}\right)+\cdots=O+F_{1}(O)+F_{2}\left(A_{1}\right)+F_{3}\left(A_{2}\right)+\cdots \cdot$

Continuing thus, it is found that

$\dagger$ The values on the lines $x$ (or $y)=s_{\nu}{ }^{r}$ (the $s_{\nu}{ }^{r}$ being points of $E^{r}$ ) are of no importance for our purposes.

$\ddagger$ That is, values for which the conditions specified in $\$ 9$ hold.

$\S$ H. Lebesgue, Lę̧ons sur l'Intégration et la Recherche des Fonctions Primitives, Paris, 1928, p. 315.

$\|$ That is, if $h$ represents a point of $H$, the corresponding point of $F_{i}(H)$ will be represented by $1 /(i+1)+(1 / i-1 /(i+1)) h$. 


$$
E^{n}=O+F_{n}(O)+F_{n+1}\left(A_{1}\right)+F_{n+2}\left(A_{2}\right)+\cdots
$$

for $n=1,2, \cdots(n<\omega)$. It is noted that $E^{n}$ is in the closed interval $(0,1 /(n+1))$, the point $1 /(n+1)$ being an isolated point of $E^{n}$.

To cover $E$, as defined by (10.5a), by a finite number of sets of intervals, following the scheme which we established previously, we proceed as follows. The set $\Delta^{1}\left(\delta_{1}\right)$ will consist of the single interval

$$
\Delta_{1}^{1}\left(\delta_{1}\right)=.\left(0, \delta_{1}\right)
$$$$
\left(0<\delta_{1}<1 / 2\right),
$$

where $\delta_{1}$ is not coincident with any of the points of $E$. The set (10.6a) "covers" $E^{\omega}$. Corresponding to $\delta_{1}$ there exists a number $j=j\left(\delta_{1}\right)\left[j\left(\delta_{1}\right) \rightarrow \infty\right.$ with $\left.1 / \delta_{1}\right]$, such that every point of each of the sets

$$
E^{i+1}, E^{i+2}, \cdots
$$

is interior to* $\left(0, \delta_{1}\right)$, while the set $E^{i}$ has points not in $\Delta_{1}{ }^{1}\left(\delta_{1}\right)$; that is, there are points of $E^{i}$ in the interval $\left(\delta_{1}<x<1\right)$. In view of the statement subsequent to (10.6) one clearly has

that is,

$$
\frac{1}{j+2}<\delta_{1}<\frac{1}{j+1}
$$

$$
\frac{1}{\delta_{1}}-2<j<\frac{1}{\delta_{1}}-1 . \dagger
$$

The points of $E^{j}$ exterior to $\Delta^{1}\left(\delta_{1}\right)$ will be $F_{j}(O)$, that is $1 /(j+1)$, and those points of $F_{j+1}\left(A_{1}\right)$ which are to the right of $\delta_{1}$. The points of $A_{1}$ being $0,1 / 2,1 / 3, \cdots$, those of $F_{j+1}\left(A_{1}\right)$ will be

$$
\frac{1}{j+2}+l_{j+1^{\nu}}\left(l_{j+1}=1 /(j+1)-1 /(j+2)\right),
$$

where $\nu=0,1 / 2,1 / 3, \cdots$. Accordingly it is concluded that the points of $E^{i}$, exterior to $\Delta^{1}\left(\delta_{1}\right)$, will consist either of the single point $1 /(j+1)$, or of the $m^{1}$ $(>1)$ points

$$
\begin{aligned}
& s_{1}^{j}=\frac{1}{j+1}, \quad s_{2}^{j}=\frac{1}{j+2}+\frac{1}{2} l_{j+1}, \\
& s_{3}^{j}=\frac{1}{j+2}+\frac{1}{3} l_{j+1}, \cdots, \quad s_{m 1}^{j}=\frac{1}{j+2}+\frac{1}{m^{1}} l_{j+1},
\end{aligned}
$$

where $m^{1}=m\left(\delta_{1}\right)$ and

* According to previously made conventions, "interior to" here means "in the interval $a \leqq x<\delta_{1}$."

$\dagger$ Given an "admissible" $\delta_{1}$ this defines the integer $j$ uniquely. 


$$
\frac{l_{j+1}}{\delta_{1}-(j+2)^{-1}}-1<m^{1}<\frac{l_{j+1}}{\delta_{1}-(j+2)^{-1}} .
$$

In succession "covering" sets $\Delta^{r}\left(\delta_{r}\right)(r=2,3, \cdots, j+2)$ are obtained, with $\Delta^{r}\left(\delta_{r}\right)$ consisting of intervals

$$
\begin{aligned}
\Delta_{\nu}^{r}\left(\delta_{r}\right)= & \left(s_{\nu}^{j+2-r}-\delta_{r}, s_{\nu}^{j+2-r}+\delta_{r}\right) \\
& {\left[\nu=1,2, \cdots, m^{1} ; m^{1}=m\left(\delta_{1}, \delta_{2}, \cdots, \delta_{r-1}\right)\right], }
\end{aligned}
$$

so that $E^{j}$ is interior to $\Delta^{1}\left(\delta_{1}\right)+\Delta^{2}\left(\delta_{2}\right)$ (a finite number of points of $E^{j}$ in $\left.\Delta^{2}\left(\delta_{2}\right)\right), E^{j-1}$ is interior to $\Delta^{1}\left(\delta_{1}\right)+\cdots+\Delta^{3}\left(\delta_{3}\right)$ (a finite number of points of $E^{i-1}$ in $\left.\Delta^{3}\left(\delta_{3}\right)\right)$ and so on, with $E=E^{0}$ interior to the set

$$
\Delta^{1}\left(\delta_{1}\right)+\cdots+\Delta^{i+2}\left(\delta_{j+2}\right),
$$

only a finite number of points of $E$ lying in $\Delta^{j+2}\left(\delta_{j+2}\right) .^{*}$

Let

$$
\begin{aligned}
& K(x, y)=g(x) \\
& K(x, y)=g(y)
\end{aligned}
$$$$
\text { (for } 0 \leqq y<x) \text {, }
$$$$
\text { (for } 0 \leqq x<y \text { ), }
$$

where $g(x)$ is defined as follows. The set $E$ of (10.5a) being closed nondense, the complementary set $(0,1)-E$ will consist of a denumerable infinity of open (except at 1), nonoverlapping intervals

$$
\left(a_{i}, b_{i}\right) \quad\left(0 \leqq a_{i}<b_{i} \leqq 1 ; i=1,2, \cdots\right),
$$

some of them being adjacent. We let $g(x)=0$ for $3 / 4 \leqq x \leqq 1$ and in the other intervals $(10.8 \mathrm{~b})$ we take

$$
\begin{aligned}
g(x) & =0 & \left(\text { for } a_{i}\right. & \left.<x<\left(a_{i}+b_{i}\right) / 2\right), \\
g^{2}(x) & =\frac{c_{i}}{b_{i}{ }^{2}-x^{2}} & \left(\text { for }\left(a_{i}+b_{i}\right) / 2\right. & \left.\leqq x<b_{i} ; c_{i}>0\right) . \dagger
\end{aligned}
$$

In accordance with Definition 10.1 related to $K(x, y)$ will be the kernel

$$
\begin{aligned}
& K^{\delta_{1}}, \cdots, \delta_{j+2}(x, y)=g^{\delta_{1}} \cdots, \delta_{j+1}(x) \\
& \mathrm{Y}^{\delta_{1}}, \cdots, \delta_{j+2}(x, y)=g^{\delta_{1}}, \cdots, \delta_{j+1}(y)
\end{aligned}
$$$$
(0 \leqq y<x),
$$

where

$$
\begin{aligned}
& g^{\delta_{1}}, \cdots, \delta_{j+2}(x)=0 \\
& g^{\delta_{1}, \cdots, \delta_{j+2}}(x)=g(x)
\end{aligned}
$$

$$
\begin{array}{r}
{\left[\text { for } x \text { in } \sum_{\nu=1}^{j+2} \Delta^{\nu}\left(\delta_{\nu}\right)\right],} \\
\text { for } \left.x \text { in }(0,1)-\sum_{\nu=1}^{j+2} \Delta^{\nu}\left(\delta_{\nu}\right)\right] .
\end{array}
$$

* The numbers $m^{1}$ involved in (10.7) can be specified by inequalities in terms of $\delta_{1}, \cdots, \delta_{r-1}$ in succession, making use of the fact that the $\delta_{\nu}$ are chosen so as to secure "covering" in our sense.

$\dagger$ The definition for $x$ equal to a number corresponding to a point of $E$ is immaterial. 
Now the $G_{i}$ in (10.8c) are points in $E$ and they are interior to the finite number of nonadjacent nonoverlapping intervals constituting (10.7a). The set

$$
(0,1)-\sum_{1}^{j+2} \Delta^{v}\left(\delta_{v}\right)
$$

will consist of a finite number of open intervals $\Gamma$. Any particular interval $\Gamma$ will be, together with its end points, interior to some interval $\left(a_{i}, b_{i}\right)$. In view of $(10.8 \mathrm{c})$ and $(10.9 \mathrm{~b})$

$$
\left|g^{\delta_{1}, \cdots, \delta_{j+2}(x)}\right|^{2} \leqq \frac{\left|c_{i}\right|}{\left|b_{i}^{2}-x^{2}\right|} \quad(x \text { in interval } \Gamma) .
$$

With $b_{i}$ exterior to the given interval $\Gamma,\left|g^{\delta_{1}, \cdots, \delta_{j+2}(x)}\right|$ will be uniformly bounded in this interval. Hence this function will be uniformly bounded in the total set of intervals constituting $\Gamma$, inasmuch as the number of these intervals is finite. On taking account of the first relation (10.9b) it is finally concluded that

$$
\left|g^{\delta_{1}}, \cdots, \delta_{j+2}(x)\right| \leqq \beta\left(\delta_{1}, \cdots, \delta_{j+2}\right) \quad(0 \leqq x \leqq 1),
$$

where the second member is independent of $x$ and is finite whenever $\delta_{1}, \cdots, \delta_{j+2}$ have positive "admissible" values. In consequence of (10.9), (10.9a), and (10.9d) $K^{\delta_{1}}, \cdots, \delta_{i+2}(x, y) \subset L_{2}$ (in $x, y$; for $\left.0 \leqq x, y \leqq 1\right)$. Thus, (10.8), (10.8a) furnishes an example of a kernel $K(x, y) \subset H_{\omega+1}$. Now, we recall that convergence of

$$
\left.\int_{0}^{1} K^{2}(x, y) d y \quad \text { (almost all } x \text { on }(0,1)\right)
$$

would imply that $K(x, y)$ is essentially of one of the classes of kernels considered by Carleman. This, however, is not the case. In fact, the integral (10.10), if convergent, could be written as

$$
x g^{2}(x)+\int_{x}^{1} g^{2}(y) d y .
$$

For every $0 \leqq x<3 / 4$ the interval $(x, 1)$ would contain at least one point $b_{i}$ (cf. (10.8c)). The presence of such an infinite discontinuity implies that there exists no integral (10.10a).

On determining in succession the limits

$$
\begin{aligned}
& \lim _{\delta_{j+2}} K^{\delta_{1}} \cdots, \delta_{j+2}(x, y)=K^{\delta_{1}}, \cdots, \delta_{j+1}(x, y), \\
& \lim _{\delta_{i+1}} K^{\delta_{1}}, \cdots, \delta_{j+1}(x, y)=K^{\delta_{1}}, \cdots, \delta_{j}(x, y), \cdots, \\
& \lim _{\delta_{2}} K^{\delta_{1}, \delta_{2}}=K^{\delta_{1}}(x, y), \quad \lim _{\delta_{1}} K^{\delta_{1}}(x, y)=K(x, y) \text {, }
\end{aligned}
$$


it is observed that

$$
\begin{aligned}
& K^{\delta_{1}}, \cdots, \delta_{j+1}(x, y) \subset H_{1}, \quad K^{\delta_{1}}, \cdots, \delta_{j}(x, y) \subset H_{2}, \cdots, \\
& K^{\delta_{1}, \delta_{2}}(x, y) \quad \subset H_{j}, \quad K^{\delta_{1}}(x, y) \quad \subset H_{j+1} .
\end{aligned}
$$

The latter relationship above implies that $K(x, y)$ is a limit of a simply infinite sequence of kernels each of which belongs to a class $H_{\nu}(\nu<\omega)$. It is of interest to assure that

$$
\left.\int_{0}^{1}\left|K^{\delta_{1}}(x, y)\right|^{2} d y \quad \text { (all "admissible" } \delta_{1}>0\right)
$$

diverges, since in the contrary case $K^{\delta_{1}}(x, y)$ would be essentially of Carleman's type and $K(x, y)$, itself, would be of rank two. Now

$$
K^{\delta_{1}}(x, y)= \begin{cases}0 & \left(0 \leqq x \leqq \delta_{1} ; 0 \leqq y<x\right), \\ 0 & \left(0 \leqq y \leqq \delta_{1} ; 0 \leqq x<y\right),\end{cases}
$$

and $K^{\delta_{1}}(x, y)=K(x, y)$ at the other points of $0 \leqq x, y \leqq 1$. $^{*}$ The integral (10.11b), if it exists, is of the form (10.10a), where $g(x)$ is replaced by $g^{\delta_{1}}(x)$,

$$
g^{\delta_{1}}(x)= \begin{cases}0 & \left(0 \leqq x \leqq \delta_{1}\right), \\ g(x) & \left(\delta_{1}<x \leqq 1\right) .\end{cases}
$$

Thus, the integral (10.11a) will exist if and only if

$$
\int_{x}^{1}\left|g^{\delta_{1}}(y)\right|^{2} d y
$$

exists. The expression last displayed is identical with

$$
\left.\int_{\delta_{1}}^{1} g^{2}(y) d y \quad \text { (for } 0 \leqq x \leqq \delta_{1}\right),
$$

which diverges for $\delta_{1}<3 / 4$ (cf. the statement subsequent to (10.10a)). When $\delta_{1}<x<1$

$$
\int_{x}^{1}\left|g^{\delta_{1}}(y)\right|^{2} d y=\int_{x}^{1} g^{2}(y) d y
$$

this diverges for reasons previously given with reference to (10.10a). Hence it is inferred that $(10.11 \mathrm{~b})$ diverges, as stated. It remains to make certain that $K(x, y)$ does not belong to a class $H_{n}$, where $n<\omega$. In fact, suppose $K(x, y) \subset H_{n}$. Then by Definition $2.2 \dagger$ there exists a set $\bar{E}=\bar{E}^{0}$, with

* In accordance with certain previous remarks, the values of $K(x, y)$ on lines $x=$ number corresponding to a point of $E$ are immaterial.

$\dagger$ To conform with the present notation, $\delta_{\nu}, \Delta^{\nu}$, in Definition 2.2 , are replaced by $\delta_{\nu+1}, \Delta^{\nu+1}$, respectively. 


$$
\bar{E}^{n-1}=\left(\bar{s}_{1}, \cdots, \bar{s}_{k}\right),
$$

and sets $\Delta_{1}{ }^{\nu}\left(\bar{\delta}_{\nu}\right)(\nu=1, \cdots, n)$ of intervals such that the points of $\bar{E}^{n-i}$ are interior to $\Delta_{1}^{1}\left(\bar{\delta}_{1}\right)+\cdots+\Delta_{1}^{i}\left(\bar{\delta}_{i}\right)(i=1, \cdots, n)$, the only points of $\bar{E}^{n-i}$ in $\Delta_{1}^{i}\left(\bar{\delta}_{i}\right)$ being the centers

$$
\bar{s}_{\nu}^{n-\imath} \quad\left[\nu=1, \cdots, m^{1} ; m^{1}=m\left(\bar{\delta}_{1}, \bar{\delta}_{2}, \cdots, \bar{\delta}_{i-1}\right)\right]
$$

of the constituent intervals of $\Delta_{1}^{i}\left(\bar{\delta}_{i}\right)$. Moreover,

$$
K^{\bar{\delta}_{1}}, \cdots, \bar{\delta}_{n}(x, y) \subset L_{2}
$$

here the first member equals $K(x, y)$, except for $x$ in $G=\Delta_{1}{ }^{1}\left(\bar{\delta}_{1}\right)+\cdots+\Delta_{1}{ }^{n}\left(\bar{\delta}_{n}\right)$ $(0 \leqq y<x)$ and also for $y$ in $G(0 \leqq x<y)$, where the first member of $(10.12)$ is zero. The complement of $G$ could not contain an interval $(0, h)(h>0)$, in fact, if it did one would have

$$
\int_{0}^{h} \int_{0}^{h}\left|K^{\bar{\delta}_{1}, \cdots, \bar{\delta}_{n}}(x, y)\right|^{2} d x d y=\int_{0}^{h} \int_{0}^{h} K^{2}(x, y) d x d y .
$$

The integrand last displayed has an infinity of infinite discontinuities within the field of integration, each of which, alone, would suffice to secure divergence of the integral in question. Thus (10.12) does not hold for all "admissible" sufficiently small $\bar{\delta}_{\nu}$, unless $G$ contains an interval $(0, h)(h>0)$ for every "admissible" choice of $\bar{\delta}_{1}, \cdots, \bar{\delta}_{n} *$ Hence the point 0 must be the center (that is, end point, in this case) of an interval of $G$. Suppose this point is the center of an interval $\Gamma_{1}$ of the set $\Delta_{1}{ }^{r}\left(\delta_{r}\right)$. We recall that having made an "admissible" choice of $\bar{\delta}_{1}, \bar{\delta}_{2}, \cdots, \bar{\delta}_{r-1}$, the choice of $\bar{\delta}_{r}, \cdots, \bar{\delta}_{n}$ depends on that of $\bar{\delta}_{1}, \cdots, \bar{\delta}_{r-1}$; however, $\bar{\delta}_{r}$ may be taken arbitrarily small. Thus, with $\bar{\delta}_{r}$ suitably small, there will exist an interval $\Gamma_{2}$, adjacent to and nonoverlapping with $\Gamma_{1}$, which will be in the complement of $G$ and in which $K(x, y) \cdot$ will have infinite discontinuities (cf. (10.8)-(10.8c)); on the other hand,

$$
K^{\bar{\delta}_{1}}, \cdots, \bar{\delta}_{n}(x, y)=K(x, y)
$$

for $x$ in $\Gamma_{2}$ and $0 \leqq y \leqq 1$ and also for $y$ in $\Gamma_{2}$ and $0 \leqq x \leqq 1$. The presence of the above discontinuities implies that (10.12) does not hold, as stated. Thus, our kernel $K(x, y)$, as given by (10.8)-(10.8c), is of the transfinite class $H_{\omega+1}$ and does not belong to any class of index less than $\omega+1$.

Following the procedure indicated from (10.8) to the italicized statement above, obvious generalizations can be made regarding existence and construction of kernels of various transfinite classes.

11. Results for classes $H_{\beta}$. Let $K(x, y) \subset H_{\beta}$ where $\beta=\omega+p(0<p<\omega)$.

${ }^{*} h$ may depend on $\bar{\delta}_{1}, \cdots, \bar{\varepsilon}_{n}$. 
For the corresponding set $E$ we shall have

$$
E^{\omega+p-1}=\left(s_{\nu}\right) \quad(\nu=1, \cdots, k) .
$$

Sets $\Delta^{v}\left(\delta_{v}\right)$ each consisting of a finite number of intervals are constructed so that

$$
\begin{gathered}
E^{\omega+p-1} \subset \Delta^{1}\left(\delta_{1}\right), \quad E^{\omega+p-2} \subset \Delta^{1}\left(\delta_{1}\right)+\Delta^{2}\left(\delta_{2}\right), \cdots, \\
E^{\omega+1} \subset \Delta^{1}\left(\delta_{1}\right)+\cdots+\Delta^{p-1}\left(\delta_{p-1}\right), \quad E^{\omega} \subset \Delta^{1}\left(\delta_{1}\right)+\cdots+\Delta^{p}\left(\delta_{p}\right)=\Gamma,
\end{gathered}
$$

where $G \subset H$ signifies that every point of $G$ is an interior point of $H$. In (11.1a) the only points $E^{\omega+p-i}$ which are in $\Delta^{i}\left(\delta_{i}\right)$ are the centers

$$
s_{\nu}^{\omega+p-i} \quad\left[\nu=1, \cdots, m^{1} ; m^{1}=m\left(\delta_{1}, \cdots, \delta_{i-1}\right)\right]^{*}
$$

of the constituent intervals of $\Delta^{i}\left(\delta_{i}\right)$; this assertion is made for $i=1, \cdots, p$. Furthermore, for some $j=j\left(\delta_{1}, \cdots, \delta_{p}\right)$

while

$$
E^{i+\nu} \subset \Gamma \quad[\nu=1,2, \cdots ; \text { cf. (11.1a) }],
$$

$E^{i} \pitchfork \Gamma$

the points of $E^{i}$, not in $\Gamma$, being finite in number,

$$
s_{1}^{j}, \cdots, s_{m^{1}}^{j} \quad\left(m^{1}=m\left(\delta_{1}, \cdots, \delta_{p}\right)\right) .
$$

Further sets $\Delta^{\nu}\left(\delta_{\nu}\right)(\nu=p+1, p+2, \cdots, p+j+1)$ of intervals are constructed so that

$$
\begin{aligned}
E^{i} & \subset \Gamma+\Delta^{p+1}\left(\delta_{p+1}\right), \quad E^{j-1} \subset \Gamma+\Delta^{p+1}\left(\delta_{p+1}\right)+\Delta^{p+2}\left(\delta_{p+2}\right), \cdots, \\
E^{1} & \subset \Gamma+\Delta^{p+1}\left(\delta_{p+1}\right)+\cdots+\Delta^{p+i}\left(\delta_{p+j}\right), \\
E^{0}= & E \subset \Gamma+\Delta^{p+1}\left(\delta_{p+1}\right)+\cdots+\Delta^{p+j+1}\left(\delta_{p+j+1}\right),
\end{aligned}
$$

the symbol $\subset$ having the same meaning as in (11.1a), the only points of $E^{j-r}$ in $\Delta^{p+r+1}\left(\delta_{p+r+1}\right)$ being the centers

$$
s_{1}^{j-r}, s_{2}^{j-r}, \cdots, s_{m 1}^{j-r} \quad\left[m^{1}=m\left(\delta_{1}, \cdots, \delta_{p+r}\right)\right]
$$

of the intervals constituting $\Delta^{p+r+1}\left(\delta_{p+r+1}\right)(r=0,1, \cdots, j)$.

According to Definition (10.1), associated with $K(x, y)$ will be the function

$$
K^{\delta_{1}, \delta_{2}, \cdots, \delta_{q}}(x, y) \quad(q=p+j+1),
$$

satisfying the conditions of that Definition. In succession we define the limits

* For $i=1, m^{1}=k$. 


$$
\lim _{\delta_{p+j+1}} K^{\delta_{1}}, \cdots, \delta_{p+j+1}(x ; y)=K^{\delta_{1}} \cdots, \delta_{p+j}(x, y),
$$

$$
\begin{aligned}
& \lim _{\delta_{p+j}} K^{\delta_{1}}, \cdots, \delta_{p+j}(x, y)=K^{\delta_{1}}, \cdots, \delta_{p+j-1}(x, y), \cdots, \\
& \lim _{\delta_{p+1}} K^{\delta_{1}, \cdots, \delta_{p+1}}(x, y)=K^{\delta_{1}} \cdots, \delta_{p}(x, y),
\end{aligned}
$$

where, in particular,

$$
\begin{array}{rlr}
K^{\delta_{1}}, \cdots, \delta_{p}(x, y) & =0 \\
& =0 & \text { [for } x \text { in } \Gamma ; 0 \leqq y \leqq 1],
\end{array}
$$

and $K^{\delta_{1}}, \cdots, \delta_{p}(x, y)=K(x, y)$ at the other points of $0 \leqq x, y \leqq 1$. It is essential to note that the kernels involved in (11.3a) are all of finite ranks. The next limiting process

$$
\lim _{\delta_{p}} K^{\delta_{1}}, \cdots, \delta_{p}(x, y)=K^{\delta_{1}}, \cdots, \delta_{p-1}(x, y)
$$

is essentially distinct from those of (11.3a); it yields a kernel which may be actually of transfinite rank.* Further limiting processes will yield

$$
\begin{gathered}
\lim _{\delta_{p-1}} K^{\delta_{1}}, \cdots, \delta_{p-1}(x, y)=K^{\delta_{1}}, \cdots, \delta_{p-2}(x, y), \cdots, \\
\lim _{\delta_{2}} K^{\delta_{1}, \delta_{2}}(x, y)=K^{\delta_{1}}(x, y), \quad \lim _{\delta_{1}} K^{\delta_{1}}(x, y)=Y^{0}(x, y)=K(x, y) .
\end{gathered}
$$

Clearly

$$
\left|K^{\delta_{1}}, \cdots, \delta_{\nu+1}(x, y)\right| \leqq\left|K^{\delta_{1}}, \cdots, \delta_{\nu}(x, y)\right| \quad(\nu=0,1, \cdots, p+j) .
$$

Corresponding to the last member of $(11.3 \mathrm{a})$ we form the equations

$$
\phi^{\delta_{1}}, \cdots, \delta_{p}(x)-\lambda \int_{a}^{b} K^{\delta_{1}}, \cdots, \delta_{p}(x, y) \phi^{\delta_{1}} \cdots, \delta_{p}(y) d y=f(x) \quad\left(f(x) \subset L_{2}\right),
$$

$$
\phi^{\delta_{1}}, \cdots, \delta_{p}(x)-\lambda \int_{a}^{b} K^{\delta_{1}}, \cdots, \delta_{p}(x, y) \phi^{\delta_{1}}, \cdots, \delta_{p}(y) d y=0 .
$$

Inasmuch as $K^{\delta_{1}}, \cdots, \delta_{p}(x, y)\left(\delta_{1}>0, \cdots, \delta_{p}>0\right)$ is of some class $H_{n}(n<\omega)$ the results of Theorem 4.1 will be applicable to the equations (11.5), (11.6).

Thus, corresponding to $K^{\delta_{1}}, \cdots, \delta_{p}(x, y)$ there exists a function $\Omega^{\delta_{1}, \cdots, \delta_{p}}(x, y)$ such that

$$
\begin{aligned}
& \operatorname{Var} . \Omega^{\delta_{1}}, \cdots, \delta_{p}(x, y \mid \lambda) \leqq[(x-a)(y-a)]^{1 / 2}, \Omega^{\delta_{1}} \cdots, \delta_{p}(x, y \mid 0)=0, \\
& \left|\Omega^{\delta_{1}}, \cdots, \delta_{p}\left(x^{1}, y^{1} \mid \lambda\right)-\Omega^{\delta_{1}}, \cdots, \delta_{p}(x, y \mid \lambda)\right| \\
& \leqq(b-a)^{1 / 2}\left(\left|y^{1}-y\right|^{1 / 2}+\left|x^{1}-x\right|^{1 / 2}\right) ;
\end{aligned}
$$

* That is, in some cases $K^{\delta_{1}}, \cdots, \delta_{p-1}(x, y)$ will belong to $H_{\omega+1}$ and will be not of class $H_{\nu}(\nu<\omega)$. 
this function may be discontinuous in $\lambda$ for a denumerable infinity of real values $\lambda$. In view of (11.7), (11.7a), application of the "Compactness Theorem" ( $\$ 1)$, with respect to $\delta_{p}$, leads to the conclusion that the limit

(11.8) $\lim _{\delta_{p, r}} \Omega^{\delta_{1}}, \cdots, \delta_{p}(x, y \mid \lambda)=\Omega^{\delta_{1}}, \cdots, \delta_{p-1}(x, y \mid \lambda) \quad$ [suitable $\delta_{p, r} ; \lim \delta_{p, r}=0$ ] exists and satisfies

$$
\begin{aligned}
& \text { Var. } \Omega^{\delta_{1}}, \cdots, \delta_{p-1}(x, y \mid \lambda) \leqq[(x-a)(y-a)]^{1 / 2}, \Omega^{\delta_{1}}, \cdots, \delta_{p-1}(x, y \mid 0)=0 \\
& \left|\Omega^{\delta_{1}}, \cdots, \delta_{p-1}\left(x^{1}, y^{1} \mid \lambda\right)-\Omega^{\delta_{1}}, \cdots, \delta_{p-1}(x, y \mid \lambda)\right| \leqq \operatorname{second~member~of~}(11.7 \mathrm{a})
\end{aligned}
$$

moreover, $\Omega^{\delta_{1}} \cdots, \delta_{p-1}(x, y \mid \lambda)$ will have the same descriptive properties as $\Omega^{\delta_{1}}, \cdots, \delta_{p}(x, y \mid \lambda)$. Continuing in this manner along the lines of $\S 4$ we conclude that the results of Theorem 4.1 all hold for the kernel (which is generally of transfinite rank) $K^{\delta_{1}}, \cdots, \delta_{p-1}(x, y)$. Continuing the reasoning of the type employed before, passing to the limit, it is established that results of such type hold for $K(x, y)$, itself. Finally, by transfinite induction the following Theorem is established.

TheOREM 11.1. Let $K(x, y)$ be a kernel of class $H_{\beta}$, where $\beta$ is any number of the first or second class (Definition 10.1). With respect to this kernel all the results (stated in appropriate form) of Theorem 4.1 will hold.

Many more significant results for kernels $H_{\beta}(\beta>\omega)$ can be obtained whenever with the kernel in question one may associate an operator $L$ satisfying the following definition.

Definition 11.1. A linear operator $L_{x}(\xi \mid h(x))$ ( $\xi$ a parameter) will be said to be associated with $K(x, y) \subset H_{\beta}(\beta>\omega)$ (cf. Definition 10.1) if

$$
\begin{gathered}
L_{x}(\xi \mid K(x, y)) \subset L_{2} \\
\left|L_{x}\left(\xi \mid K^{\delta_{1}}, \cdots, \delta_{q}(x, y)\right)\right|<\gamma(\xi \mid y),
\end{gathered}
$$

with $\gamma(\xi \mid y) \subset L_{2}$ (in $y$ ), the function $\gamma(\xi \mid y)$ being independent of $\delta_{1}, \cdots, \delta_{q}$;

$$
\begin{aligned}
L_{x}\left(\xi \mid K^{\delta_{1}}, \cdots, \delta_{q}(x, y)\right) & \underset{\delta_{q}}{\rightarrow} L_{x}\left(\xi \mid K^{\delta_{1}}, \cdots, \delta_{q-1}(x, y)\right) \\
& \underset{\delta_{q-1}}{\longrightarrow} L_{x}\left(\xi \mid K^{\delta_{1}, \cdots, \delta_{q-2}}(x, y)\right) \rightarrow \cdots \underset{\delta_{1}}{\rightarrow} L_{x}(\xi \mid K(x, y)) ;
\end{aligned}
$$

(11.12) $\lim _{\nu} L_{x}\left(\xi \mid f_{\nu}(x)\right)=L_{x}(\xi \mid f(x))\left[\right.$ when $f_{\nu}(x)$, included in $L_{2}, \rightarrow f(x)$ weakly];

$$
\int_{a}^{b} L_{x}\left(\xi \mid K^{\delta_{1}}, \cdots, \delta_{q}(x, y)\right) \phi(y) d y=L_{x}\left(\xi \mid \int_{a}^{b} K^{\delta_{1}, \cdots, \delta_{q}}(x, y) \phi(y) d y\right)
$$

for all $\phi(x) \subset L_{2}$. Here $\delta_{1}, \cdots, \delta_{q}$ are the numbers referred to in Definition 10.1 . Before proceeding further it is essential to give an example of a kernel of 
transfinite rank, which is at the same time not of any finite rank and with which one can "associate" in the sense of Definition 11.1, an operator $L$. For this purpose consider the kernel $K(x, y)$ defined by (10.8)-(10.8c) (cf. text from (10.5) to $(10.8 \mathrm{c})) ; K(x, y) \subset H_{\omega+1}$ and $K(x, y)$ does not belong to any $H_{\nu}$ with $\nu<\omega$. The associated operator will be taken of the form

$$
L_{x}(\xi \mid h(x))=\int_{0}^{1} G(\xi \mid x) h(x) d x,
$$

where

(11.14a) $\quad G(\xi \mid x)=0$

$(3 / 4 \leqq x \leqq 1)$,

(11.14b) $\quad G(\xi \mid x)=c_{i}^{-1 / 2}\left(b_{i}{ }^{2}-x^{2}\right)^{1 / 2} w_{i}(\xi \mid x) \quad\left(\gamma_{i}=\left(a_{i}+b_{i}\right) / 2 \leqq x<b_{i}\right)$,

(11.14c) $\quad G(\xi \mid x)=-G_{i}\left(\xi \mid a_{i}+b_{i}-x\right)$,

$\left(a_{i}<x \leqq \gamma_{i}\right)$,

the $a_{i}$ and $b_{i}$ being the numbers from $(10.8 \mathrm{~b})$,

$$
0 \leqq w_{i}(\xi \mid x) \leqq H, \quad w_{i}(\xi \mid x) \subset L_{1}
$$

(in $\left.x ; \gamma_{i} \leqq x<b_{i}\right)$

the function $w_{i}(\xi \mid x)$ being monotone non-increasing in $x$ on $\left(\gamma_{i}, b_{i}\right)$.

By (11.14b) and (11.14d)

$$
|G(\xi \mid x)|^{2} \leqq \frac{H^{2}}{c_{i}}\left(b_{i}{ }^{2}-\gamma_{i}^{2}\right) \quad\left(\gamma_{i} \leqq x<b_{i}\right),
$$

and, in view of the symmetry relation $(11.14 \mathrm{c})$,

$$
|G(\xi \mid x)|^{2} \leqq \frac{H^{2}}{c_{i}}\left(b_{i}{ }^{2}-\gamma_{i}^{2}\right) \quad\left(a_{i}<x<b_{i}\right) .
$$

Thus

$$
\begin{aligned}
\int_{a}^{b}|G(\xi \mid x)|^{2} d x & =\sum_{i} \int_{a_{i}}^{b_{i}}|G(\xi \mid x)|^{2} d x \\
& \leqq H^{2} \sum_{i} \frac{1}{c_{i}}\left(b_{i}{ }^{2}-\gamma_{i}{ }^{2}\right)\left(b_{i}-a_{i}\right)=H^{2} S .
\end{aligned}
$$

Herewith we choose the $c_{i}$ so that the series $S$ of the last member in (11.15a) converges.* Accordingly,

$$
G(\xi \mid x) \subset L_{2} \quad(\text { in } x ; 0 \leqq x \leqq 1) .
$$

In consequence of (11.14), (10.8), and (10.8a),

$$
\begin{gathered}
L_{x}(\xi \mid K(x, y))=\beta(\xi \mid y)+\alpha(\xi \mid y), \\
\beta(\xi \mid y)=g(y) \int_{0}^{y} G(\xi \mid x) d x, \quad \alpha(\xi \mid y)=\int_{y}^{1} G(\xi \mid x) g(x) d x,
\end{gathered}
$$

* This, obviously, it is always possible to do. 
$g(x)$ being defined by $(10.8 \mathrm{c})$. By (10.8c)

$$
\beta(\xi \mid y)=0 \quad\left(a_{i}<y<\gamma_{i} ; 3 / 4 \leqq y \leqq 1\right) .
$$

On the other hand, for $\gamma_{i} \leqq y<b_{i}$,

$$
\int_{0}^{y} G(\xi \mid x) d x=\int_{a_{i}}^{y} G(\xi \mid x) d x+\sum^{1} \int_{a_{j}}^{b_{j}} G(\xi \mid x) d x
$$

where the summation symbol is over the subscripts $j$, corresponding to the totality of all those numbers $b_{j}$ for which $b_{j}<b_{i}$. Now $\gamma_{j}$ is the mid-point of the interval $\left(a_{j}, b_{j}\right)$; thus, on taking account of the symmetry relation (11.14c) (for $j$ ) we conclude that

$$
\int_{a}^{b_{j}} G(\xi \mid x) d x=0
$$

Hence

$$
\int_{0}^{y} G(\xi \mid x) d x=\int_{a_{i}}^{y} G(\xi \mid x) d x \quad\left(\gamma_{i} \leqq y<b_{i}\right),
$$

so that, by $(11.14 \mathrm{c}),(11.14 \mathrm{~b})$, and in view of the monotone character of $w_{i}(\xi \mid x)$,

$$
\begin{aligned}
\int_{0}^{y} G(\xi \mid x) d x & =\int_{a_{i}}^{2 \gamma_{i}-y} G(\xi \mid u) d u=-\int_{y}^{b_{i}} G(\xi \mid u) d u \\
& =-c_{i}^{-1 / 2} \int_{y}^{b_{i}}\left(b_{i}^{2}-u^{2}\right)^{1 / 2} w_{i}(\xi \mid u) d u\left(\gamma_{i} \leqq y<b_{i}\right) ;
\end{aligned}
$$

$(11.17 \mathrm{~d})\left|\int_{0}^{y} G(\xi \mid x) d x\right| \leqq c_{i}^{-1 / 2}\left(b_{i}{ }^{2}-y^{2}\right)^{1 / 2} w_{i}(\xi \mid y)\left(b_{i}-y\right) \quad\left(\gamma_{i} \leqq y<b_{i}\right)$.

Thus, in consequence of (11.17), (10.8c), and (11.17d), it is inferred that

$$
|\beta(\xi \mid y)| \leqq w_{i}(\xi \mid y)\left(b_{i}-\gamma_{i}\right) \quad\left(\gamma_{i} \leqq y<b_{i}\right),
$$

whence by $(11.14 \mathrm{~d})$

$$
|\beta(\xi \mid y)| \leqq H\left(b_{i}-\gamma_{i}\right)<H \quad\left(\gamma_{i} \leqq y<b_{i}\right),
$$

which, together with (11.17a), implies that

$$
|\beta(\xi \mid y)|<H \quad(0 \leqq y \leqq 1) .
$$

For the function $\alpha(\xi \mid y)$, involved in (11.17), one has

$$
|\alpha(\xi \mid y)| \leqq \alpha(\xi)=\int_{0}^{1}|G(\xi \mid x)| g(x) d x=\sum_{i} \int_{a_{i}}^{b_{i}}|G(\xi \mid x)| g(x) d x,
$$


and, in consequence of (10.8c), (11.14b), (11.14d),

$(11.18 a)$

$$
\begin{aligned}
|\alpha(\xi \mid y)| \leqq \alpha(\xi) & =\sum_{i} \int_{\gamma_{i}}^{b_{i}}|G(\xi \mid x)| \frac{c_{i}^{1 / 2}}{\left(b_{i}^{2}-x^{2}\right)^{1 / 2}} d x \\
& =\sum_{i} \int_{\gamma_{i}}^{b_{i}} w_{i}(\xi \mid x) d x<H
\end{aligned}
$$

for $0 \leqq y \leqq 1$. Consideration of (11.17), (11.18), (11.18a) leads to the conclusion that condition (11.9) of Definition 11.1 holds for the case under consideration.

Consider the related kernel $K^{\delta_{1}}, \cdots, \delta_{j+2}(x, y)$ (cf. (10.9)-(10.9b)). One has

(11.19) $\quad L_{x}\left(\xi \mid K^{\delta_{1}}, \cdots, \delta_{j+2}(x, y)\right)=\beta^{\delta_{1}, \cdots, \delta_{j+2}}(\xi \mid y)+\alpha^{\delta_{1}, \cdots, \delta_{j+2}}(\xi \mid y)$,

(11.19a)

$$
\beta^{\delta_{1}}, \cdots, \delta_{j+2}(\xi \mid y)=g^{\delta_{1}}, \cdots, \delta_{j+2}(y) \int_{0}^{y} G(\xi \mid x) d x,
$$

$$
\alpha^{\delta_{1}}, \cdots, \delta_{j+2}(\xi \mid y)=\int_{y}^{1} G(\xi \mid x) g^{\delta_{1}}, \cdots, \delta_{j+2}(x) d x .
$$

We have, as can be seen from (10.9b),

$$
0 \leqq\left|g^{\delta_{1}}, \cdots, \delta_{j+2}(x)\right| \leqq g(x), \quad \lim _{\delta_{1}} \lim _{\delta_{2}} \cdots \lim _{\delta_{j+2}} g^{\delta_{1}}, \cdots, \delta_{j+2}(x)=g(x) .
$$

Thus, by (11.17) and (11.18),

$$
\left|\beta^{\delta_{1}}, \cdots, \delta_{j+2}(\xi \mid y)\right| \leqq g(x)\left|\cdot \int_{0}^{y} G(\xi \mid x) d x\right|=|\beta(\xi \mid y)|<H
$$

Also, in view of (11.18a),

$$
(0 \leqq y \leqq 1) .
$$

$$
\begin{aligned}
\left|\alpha^{\delta_{1}}, \cdots, \delta_{j+2}(\xi \mid y)\right| & \leqq \int_{0}^{1}|G(\xi \mid x)| g^{\delta_{1}} \cdots, \delta_{j+2}(\xi \mid x) d x \\
& \leqq \int_{0}^{1}|G(\xi \mid x)| g(x) d x=\alpha(\xi)<H .
\end{aligned}
$$

By virtue of (11.19), (11.19b), (11.19c) it can be asserted that condition (11.10) of Definition 11.1 holds, with $\gamma(\xi \mid y)=2{ }^{*}{ }^{*}$

To demonstrate the first relation (11.11) it will suffice to establish

$$
\begin{aligned}
& \lim _{\delta_{j+2}} \beta^{\delta_{1}}, \cdots, \delta_{j+2}(\xi \mid y)=\beta^{\delta_{1}}, \cdots, \delta_{j+1}(\xi \mid y), \\
& \lim _{\delta_{j+2}} \alpha^{\delta_{1}}, \cdots, \delta_{j+2}(\xi \mid y)=\alpha^{\delta_{1}}, \cdots, \delta_{j+1}(y),
\end{aligned}
$$

* Or, more precisely, with $\gamma(\xi \mid y)=|\beta(\xi \mid y)|+\alpha(\xi)$. 
$(11.20 a)$

$$
\beta^{\delta_{1}}, \cdots, \delta_{j+1}(\xi \mid y)=g^{\delta_{1}}, \cdots, \delta_{j+1}(y) \int_{0}^{y} G(\xi \mid x) d x,
$$

where

$$
\alpha^{\delta_{1}}, \cdots, \delta_{j+1}(\xi \mid y)=\int_{y}^{1} G(\xi \mid x) g^{\delta_{1}}, \cdots, \delta_{j+1}(x) d x
$$

$$
\begin{array}{lrl}
g^{\delta_{1}} \cdots, \delta_{j+1}(x) & =0 & {\left[x \text { in } \Delta^{1}\left(\delta_{1}\right)+\cdots+\Delta^{i+1}\left(\delta_{j+1}\right)\right],} \\
g^{\delta_{1}} \cdots, \delta_{j+1}(x) & =g(x) & {\left[x \text { in }(0,1)-\left(\Delta^{1}\left(\delta_{1}\right)+\cdots+\Delta^{j+1}\left(\delta_{j+1}\right)\right)\right] .}
\end{array}
$$

Now, the first relation (11.20) follows from (11.19a), since

$$
\lim _{\delta_{j+2}} g^{\delta_{1}}, \cdots, \delta_{j+2}(y)=g^{\delta_{1}}, \cdots, \delta_{j+1}(y) .
$$

The remaining part of (11.20) will hold if

$$
\lim _{\delta_{j+2}} \int_{y}^{1} G(\xi \mid x) g^{\delta_{1}}, \cdots, \delta_{j+2}(x) d x=\int_{y}^{1} G(\xi \mid x) g^{\delta_{1}}, \cdots, \delta_{j+1}(x) d x .
$$

In view of the inequality subsequent to (11.9a)

$$
\left|G(\xi \mid x) g^{\delta_{1}}, \cdots, \delta_{j+2}(x)\right| \leqq|G(\xi \mid x)| g(x) .
$$

The last number, here, is contained in $L_{1}$ (in $x$ ), as can be inferred from the existence of the function $\alpha(\xi)$, introduced subsequent to (11.18). On the other hand, in consequence of (11.21) the limit (as $\delta_{j+2} \rightarrow 0$ ) of the integrand in the first member of (11.22) converges to the integrand of the second member. Thus the passage to the limit under the integral sign, indicated in (11.22), is justifiable. The first condition (11.11) accordingly holds. All the other conditions (11.11) can be demonstrated in succession following the indicated procedure.

In view of (11.14) justification of (11.12) amounts to that of

$$
\lim _{\nu} \int_{0}^{1} G(\xi \mid x) f_{\nu}(x) d x=\int_{0}^{1} G(\xi \mid x) f(x) d x \quad\left(f_{\nu}(x) \rightarrow f(x) \text { weakly }\right) .
$$

This relationship, however, holds in consequence of (11.16) and of Theorem 1.4 .

Finally, demonstration of the condition (11.13) for the case under consideration is effected by noting that, in view of (10.9d), the change of order of integration, involved in the relationship

$$
\begin{aligned}
\int_{y=0}^{1} & {\left[\int_{x=0}^{1} G(\xi \mid x) K^{\delta_{1}}, \cdots, \delta_{j+2}(x, y) d x\right] \phi(y) d y } \\
= & \int_{x=0}^{1} G(\xi \mid x)\left[\int_{y=0}^{1} K^{\delta_{1}}, \cdots, \delta_{j+2}(x, y) \phi(y) d y\right] d x \quad\left(\phi(y) \subset L_{2}\right),
\end{aligned}
$$

is justifiable. 
Thus, $K(x, y)$, as defined by (10.8)-(10.8c), belongs to $H_{\omega+1}$ and does not belong to any $H_{\nu}$, with $\nu<\omega$; moreover, "associated" (in the sense of Definition 11.1) with $K(x, y)$ there is an operator $L$ of the form (11.14)-(11.14c).

Let $K(x, y)$ be any kernel of a transfinite class $H_{\beta}$ (any $\beta$ of $2 \mathrm{~d}$ class) such that with $K(x, y)$ there is "associated" an operator $L$. Then it is of interest to study the equation

$$
L_{x}(\xi \mid \phi(x))-\lambda \int_{a}^{b} L_{x}(\xi \mid K(x, y)) \phi(y) d y=L_{x}(\xi \mid f(x)) .
$$

For this purpose it is advantageous to consider the auxiliary equation

$$
\begin{array}{r}
L_{x}\left(\xi \mid \phi^{\delta_{1}} \cdots, \delta_{q}(x)\right)-\lambda \int_{a}^{b} L_{x}\left(\xi \mid K^{\delta_{1}} \cdots, \delta_{q}(x, y)\right) \phi^{\delta_{1}, \cdots, \delta_{q}}(y) d y \\
=L_{x}(\xi \mid f(x)) ;
\end{array}
$$

here the $\delta_{\nu}(\nu=1, \cdots, q)$ are the numbers involved in Definition 10.1. Of importance is also the homogeneous equation

$$
L_{x}(\xi \mid \phi(x))-\lambda \int_{a}^{b} L_{x}(\xi \mid K(x, y)) \phi(y) d y=0 .
$$

Using the results of Theorems 4.1, 5.1, 7.1, 11.1, partly by direct methods and partly by transfinite induction and following the lines which were employed previously, we arrive at the following theorem.

TheOREM 11.2. For kernels $K(x, y) \subset H_{\beta}$ (any $\beta$ of $2 d$ class) [classes $H_{\beta}$ are specified in Definition 10.1], for which there exist "associated" operators $L$ (Definition 11.1), all the results of Theorems 5.1, 7.1 will hold, if appropriately stated with respect to the equations (11.25), (11.26).

In conclusion we shall point out that if $K(x, y) \subset H_{\beta}$ ( $\beta$ possibly transfinite; Definition 10.1) and if

$$
\int_{a}^{b}\left(K(x, y)-K\left(x^{1}, y\right)\right)^{2} d y=g\left(x, x^{1}\right)
$$

exists for $x$ and $x^{1}$ in $(a, b)-E$ ( $E$ the set of $\left.\$ 9\right), g\left(x, x^{1}\right)$ being continuous in $x$ and $x^{1}$ in $(a, b)-E$, then the following will hold:

$$
\begin{aligned}
\lim _{\delta_{q, r}} \frac{\partial}{\partial x} \Omega^{\delta_{1}}, \cdots, \delta_{q, r}(x, y \mid \lambda) & =\frac{\partial}{\partial x} \Omega^{\delta_{1}, \cdots, \delta_{q-1}}(x, y \mid \lambda), \cdots, \\
\lim _{\delta_{1, r}} \frac{\partial}{\partial x} \Omega^{\delta_{1, r}}(x, y \mid \lambda) & =\frac{\partial}{\partial x} \Omega(x, y \mid \lambda) ;
\end{aligned}
$$




$$
\begin{aligned}
\lim _{\delta_{q, r}} \frac{\partial}{\partial y} \Omega^{\delta_{1}}, \cdots, \delta_{q, r}(x, y \mid \lambda) & =\frac{\partial}{\partial y} \Omega^{\delta_{1}, \cdots, \delta_{q-1}}(x, y \mid \lambda), \cdots ; \\
\lim _{\delta_{q, r}} \frac{\partial^{2}}{\partial x \partial y} \Omega^{\delta_{1}}, \cdots, \delta_{q, r}(x, y \mid \lambda) & =\frac{\partial^{2}}{\partial x \partial y} \Omega^{\delta_{1}, \cdots, \delta_{q-1}}(x, y \mid \lambda), \cdots, \\
\lim _{\delta_{1, r}} \frac{\partial^{2}}{\partial x \partial y} \Omega^{\delta_{1, r}}(x, y \mid \lambda) & =\frac{\partial^{2}}{\partial x \partial y} \Omega(x, y \mid \lambda) \quad[=\theta(x, y \mid \lambda)],
\end{aligned}
$$

provided the $\delta_{\nu, r}$ are suitably chosen. Convergence in the above relations will be uniform in any closed subset of $0 \leqq x, y \leqq b$, which has no points in common with the lines $x=I_{\nu}, y=I_{\nu}(\nu=1,2, \cdots)$. $^{*}$

To prove this fact we need only to replace in $(\mathrm{C}, \mathrm{pp} .145,146) \theta_{\delta}$ and $K_{\delta}$ by $\theta^{\delta_{1}}, \cdots, \delta_{q}$ and $K^{\delta_{1}}, \cdots, \delta_{q}$, respectively. This will yield

$$
\begin{aligned}
&\left|\theta^{\delta_{1}, \cdots, \delta_{q}}(x, y \mid \lambda)\right| {\left[\frac{1}{\left(b^{1}-a^{1}\right)^{1 / 2}}+\frac{|\lambda|}{b^{1}-a^{1}} \int_{a^{1}}^{b^{1}}\left[\int_{a}^{b}\left[K(x, s)-K\left(x^{1}, s\right)\right]^{2} d s\right]^{1 / 2} d x^{1}\right] } \\
& \cdot {\left[\frac{1}{\left(b^{1}-a^{1}\right)^{1 / 2}}+\frac{|\lambda|}{b^{1}-a^{1}} \int_{a^{1}}^{b 1}\left[\int_{a}^{b}\left[K(y, s)-K\left(x^{1}, s\right)\right]^{2} d s\right]^{1 / 2} d x^{1}\right] } \\
&\left|\theta^{\delta_{1}}, \cdots, \delta_{q}(x, y \mid \lambda)-\theta^{\delta_{1}}, \cdots, \delta_{q}\left(x^{1}, y \mid \lambda\right)\right| {\left[\left(a^{1}, b^{1}\right) \text { a closed subinterval of }(a, b)-E\right], } \\
& \leqq \lambda \mid\left[\int_{a}^{b}\left|K(x, s)-K\left(x^{1}, s\right)\right|^{2} d s\right]^{1 / 2} \\
& \cdot\left[\frac{1}{\left(b^{1}-a^{1}\right)^{1 / 2}}+\frac{|\lambda|}{b^{1}-a^{1}} \int_{a^{1}}^{b_{1}}\left[\int_{a}^{b}[K(y, s)-K(t, s)]^{2} d s\right]^{1 / 2} d t\right] .
\end{aligned}
$$

Using these inequalities, the stated result will follow with the aid of consecutive applications of the "Compactness Theorem" $(\$ 1)$.

12. Non-symmetric kernels. Let $K(x, y)$ be a kernel not necessarily symmetric. We let

$$
E_{1}, E_{2}
$$

denote reducible sets on $(a, b)$, each of the description given in the beginning of $\S 9$, with

$$
E^{\beta_{1}}=0, \quad E^{\beta_{2}}=0,
$$

where $\beta_{1}, \beta_{2}$ are non-limit numbers of the 1 st or $2 \mathrm{~d}$ class and the sets

$$
E^{\beta_{1}-1}, \quad E^{\beta_{2}-1}
$$

each have some points. In accordance with Theorem 9.1 the set $E_{1}$ will be

* $E$ consists of the points represented by the numbers $I_{\nu}$. 
"covered" by sets of intervals

$$
\Delta^{1, \nu}\left(\delta_{1, \nu}\right) \quad\left(\nu=1,2, \cdots, q_{1}<\omega\right)
$$

and the set $E_{2}$ will be "covered" by sets of intervals

$$
\Delta^{2, \nu}\left(\delta_{2, \nu}\right) \quad\left(\nu=1,2, \cdots, q_{2}<\omega\right) .
$$

Definition 12.1. A non-symmetric kernel $K(x, y)$ will be said to belong to the class $H\left(\beta_{1}, \beta_{2}\right)$ if, with the text from (12.1) to (12.2a) in view, the following is true for all "admissible" positive values $\delta_{1, \nu}, \delta_{2, \nu}$ :

$$
G(x, y)=K_{\delta_{1,1, \delta_{1}, 2}, \cdots, \delta_{1, q_{1}}}^{\delta_{2,1}, \delta_{2}, 2, \delta_{2}}, L_{2} \quad(\text { in } x, y ; \text { for } a \leqq x, y \leqq b) .
$$

Here

(12.3a) $\quad G(x, y)=0$

$\left[x\right.$ in $\left.\sum_{1}^{a_{1}} \Delta^{1, \nu}\left(\delta_{1, \nu}\right), a \leqq y \leqq b\right] ;$

(12.3b) $\quad G(x, y)=0$

[ $y$ in $\left.\sum_{1}^{a_{2}} \Delta^{2, \nu}\left(\delta_{2, \nu}\right), a \leqq x \leqq b\right] ;$

$G(x, y)=K(x, y)$

[at all other points of $a \leqq x, y \leqq b]$.

Using the well known method of Schmidt one may associate with a nonsymmetric kernel a pair of integral equations, whose kernels are symmetric. However, we shall find it more convenient to employ the device of Pérès* and, thus, associate with our kernel $T(x, y)$ a single symmetric kernel $T(x, y)$ defined as follows

$$
\begin{array}{lr}
T(x, y)=0 & (a<x, y<b), \\
T(x, y)=0 & (b<x, y<2 b-a), \\
T(x, y)=K(x, y+a-b) & (a<x<b, b<y<2 b-a), \\
T(x, y)=K(y, x+a-b) & (b<x<2 b-a, a<y<b) .
\end{array}
$$

Inasmuch as $K(x, y) \subset H\left(\beta_{1}, \beta_{2}\right)$ (Definition 12.1), one clearly has (Definition 10.1)

$$
T(x, y) \subset H_{\beta},
$$

where $\beta$ is the greater one of the numbers $\beta_{1}, \beta_{2}$. The set $E$, which according to Definition 10.1 is used in the description of a kernel of class $H_{\beta}$, consists (in the case on hand) of the points of $E_{1}$ on $(a, b)$ and of the points $b+I_{2, \nu}$ $\left[\nu=1,2, \cdots\right.$; the $I_{2, \nu}$ represent points of $\left.E_{2}\right]$.

We apply to $T(x, y)$ the results of the previous sections; this will lead to conclusions with respect to the given non-symmetric kernel $K(x, y)$.

* Volterra and Pérès, loc. cit., p. 306.

UNIVERSITY OF ILLINOIS,

URBANA, ILL. 